\title{
The Carbocation Rearrangement Mechanism, Clarified
}

Daniel J. S. Sandbeck, Daniel J. Markewich, and Allan L. L. East*

Department of Chemistry and Biochemistry, University of Regina, 3737 Wascana Parkway, Regina, SK, S4S OA2, Canada

\section{Table of Contents}

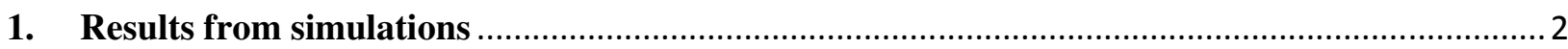

Table S1. The 80 VASP simulations (20000 1-fs steps each). Each set of 10 runs were begun with

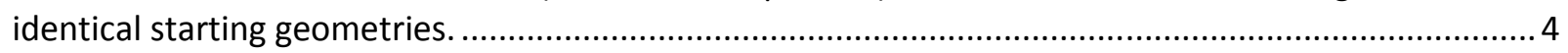

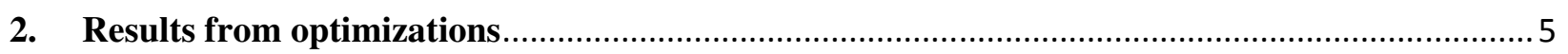

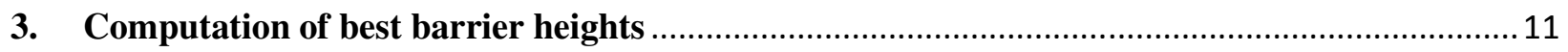

Table S2. Barrier heights ( $\mathrm{kcal} \mathrm{mol}^{-1}$ ), from CCSD(T)/aug-cc-pVTZ//PBE/6-31G(d,p) E elec computation and rigid-rotor/harmonic-oscillator thermodynamic corrections from $\mathrm{PBE} / 6-31 \mathrm{G}(\mathrm{d}, \mathrm{p})$ frequency

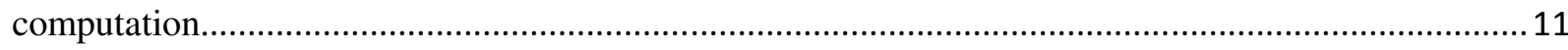

Table S3. CCSD(T)/aug-cc-pVTZ//PBE/6-31G(d,p) electronic energies (au) and PBE/6-31G(d,p)

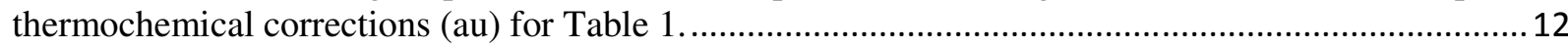

4. Appendix. Transition-state Cartesian coordinates $(\AA)$, from CCSD/6-31G(d,p) (CC) and

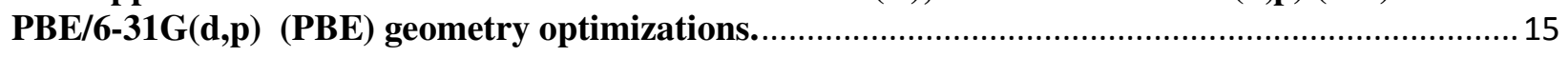

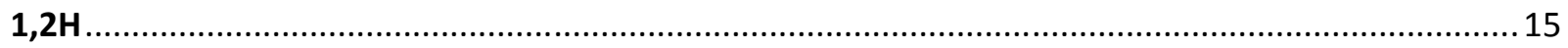

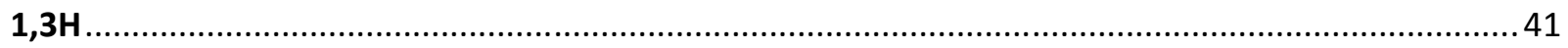

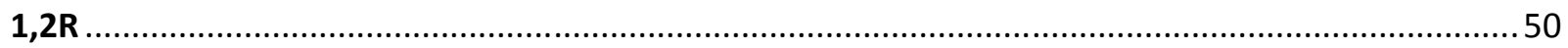

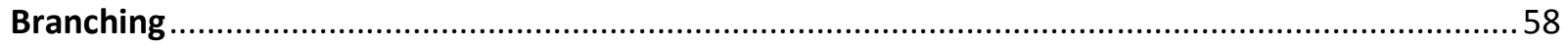

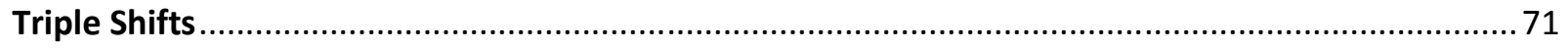

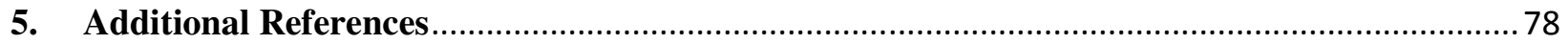




\section{Results from simulations}

Table S1 lists the 80 simulations performed.

At 600K (runs 1-20), all 20 runs remained in the "unbranched" box of Figure 4, having insufficient energy to branch (i.e. to surpass the $\delta$-PCP ${ }^{+}$-cycle "black dot" closed primary carbocation structures) in a $20 \mathrm{ps}$ timeframe. Several instances of the predicted interconversions of 9 (2-hexyl) with 8 (3-hexyl) were observed: 1,2-H shifts between nonclinal structures (9N/8N) and $1,3-\mathrm{H}$ shifts within the $\delta \mathrm{PCP}^{+}$structures $(\mathbf{9 C} / 8 \mathrm{C})$. At this temperature, $\mathrm{PCP}^{+}$structures of these secondary carbocations were short-lived but reasonably frequent: we estimated episodes of 100 fs perhaps every 500-1000 fs or so. Also observed were roughly ten instances of 1,5-Hbridging of 9N (2-hexyl ion), lasting 100-1000 fs; transannular 1,5-H-bridges are experimentally known, ${ }^{29}$ and in a simulation of acyclic $\mathrm{C}_{9} \mathrm{H}_{19}{ }^{+}$at $800 \mathrm{~K}$ such a bridge appeared for the last $40 \mathrm{ps}$ of the simulation. ${ }^{9 \mathrm{~b}}$ Both $\mathrm{PCP}^{+}$and 1,5-H-bridged forms are low-entropy closed forms whose relative stability would decrease as temperature is increased.

At $1000 \mathrm{~K}$, the runs from $\mathbf{8 C}$ and $\mathbf{9 C}$ (runs 21-40) continued to show 1,2-H and 1,3-H shifts. $\mathrm{PCP}^{+}$episodes still regularly occurred, but 1,5-H-bridged structures became very rare. At this higher temperature, 4 instances of branching via the predicted $\delta$ cycle of Figure 4 were observed: 3 cases of $\mathbf{9 C} \rightarrow \mathbf{6 C}(1,2-\mathrm{Pr}$ shift $+1,3-\mathrm{H}$ shift $)$, and 1 case of $\mathbf{8 C} \rightarrow \mathbf{5 C}(1,2-\mathrm{Et}$ shift + 1,3-H shift). These 4 branching rearrangements occurred during closed $\mathrm{PCP}^{+}$episodes that lasted 250, 70, 140, and 200 fs, respectively. We also observed a degenerate 1,4-H shift (2-hexyl to "5-hexyl"), which occurred during a $150 \mathrm{fs}$ episode of 1,4-H-bridging.

At $1000 \mathrm{~K}$, the runs from 5CA (runs 41-50) and 6CA (runs 51-60) rather quickly sunk into nearby tertiary-carbocation energy wells $(\mathbf{5} \rightarrow \mathbf{2}$ within $2.5 \mathrm{ps}$, and $\mathbf{6} \rightarrow \mathbf{3}$ within $0.5 \mathrm{ps})$, and rarely escaped these except in runs 52, 54, and 59. In all 20 runs the hexyl ion stayed in a PCP form initially and performed apparently labile 1,2-alkyl shifts before opening and falling to a tertiary form. In the case of runs 51-60, each run consistently performed $\mathbf{6 C A} \rightarrow \mathbf{5 C B}$ at $\mathrm{t}=40 \mathrm{fs}$, $\mathbf{5 C B} \rightarrow \mathbf{6 C A}$ at $\mathrm{t}=260 \mathrm{fs}$, and then $\mathbf{6} \rightarrow \mathbf{3}$ at $470-500 \mathrm{fs}$, due to dominant potential-energy initial forces that prevented significant trajectory deviations in the first picosecond. The most adventurous run (\#52) explored a significant volume of the "monobranched" box of Figure 4, achieving both 5CB and 5CA structures and some steps in the $\beta$ cycle before finishing as the tertiary ion $\mathbf{3}$. 
At $1600 \mathrm{~K}$, the runs from deep tertiary-ion energy wells $\mathbf{1 C}$ and $\mathbf{3 N}$ (runs 61-80) had sufficient energy to achieve secondary ion structures. However, $1600 \mathrm{~K}$ was also sufficient energy to achieve 5 unexpected occurrences whose probabilities were overly inflated by the high temperature: a 40-fs-long primary hexyl ion (from a forward-and-reverse 1,2-H shift) was observed in run 76, and 4 high-energy scissions occurred in runs 61 and $72\left(\mathrm{CH}_{4}\right.$ elimination), 74 ( $\beta$ scission to a primary cation), and $78\left(\mathrm{C}_{2} \mathrm{H}_{6}\right.$ elimination). All 4 scissions were of a $\mathrm{C}-\mathrm{C}$ bond $\beta$ to a charge site. More importantly, there were several predicted steps seen: the low-energy 1,2 alkyl shifts $\mathbf{6 C A} \rightarrow \mathbf{5 C B}$ ( $\delta$ cycle) and $\mathbf{5 C A} \rightarrow \mathbf{5 C A}(\beta$ cycle $)$, the double-1,2-Me shift $\mathbf{1} \rightarrow \mathbf{4 C} \rightarrow \mathbf{1}$, the low-energy $\beta$ scission from 7, unbranching 6C $\rightarrow 9 \mathrm{C}$ (run 74), and, gratifyingly, the full (and experimentally known ${ }^{26}$ ) tert $\rightarrow$ tert $\mathbf{1 C} \rightarrow \mathbf{3 C}$ conversion, here occurring via 1,2-Me, 1,3-H, and 1,2-Et shifts within a $\mathrm{PCP}^{+}$structure (run 62). The three shifts in the tert $\rightarrow$ tert conversion took a total time of $100 \mathrm{fs}$. 
Table S1. The 80 VASP simulations (20000 1-fs steps each). Each set of 10 runs were begun with identical starting geometries.

\begin{tabular}{|c|c|c|c|c|c|}
\hline Run\# & file & Interesting Events & Run\# & file & Interesting Events \\
\hline 1 & 01_600_9CC & 1,5H-brg (180 fs) & 41 & 01_1000_5CCA & $1,2 \mathrm{Et} \beta, \mathbf{5} \rightarrow \mathbf{2}$ within $2500 \mathrm{fs}$ \\
\hline 2 & 02_600_9CC & $1,3 \mathrm{H} \delta$ & 42 & 02_1000_5CCA & As above \\
\hline 3 & 03_600_9CC & $1,3 \mathrm{H} \delta$ & 43 & 03_1000_5CCA & As above \\
\hline 4 & 04_600_9CC & & 44 & 04_1000_5CCA & As above \\
\hline 5 & 05_600_9CC & & 45 & 05_1000_5CCA & As above \\
\hline 6 & 06_600_9CC & 1,5H-brg (1030 fs) & 46 & 06_1000_5CCA & As above \\
\hline 7 & 07_600_9CC & & 47 & 07_1000_5CCA & As above \\
\hline 8 & 08_600_9CC & $1,3 \mathrm{H} \delta$ & 48 & 08_1000_5CCA & As above \\
\hline 9 & 09_600_9CC & & 49 & 09_1000_5CCA & As above \\
\hline 10 & 10_600_9CC & $1,5 \mathrm{H}-\mathrm{brg}, 1,3 \mathrm{H} \delta$ & 50 & 10_1000_5CCA & As above \\
\hline 11 & 01_600_8CAB & $1,5 \mathrm{H}-\mathrm{brg}, 1,3 \mathrm{H} \delta$ & 51 & 01_1000_6CCA & $2 \times(1,2 \mathrm{Me} \delta), 6 \rightarrow 3$ within $500 \mathrm{fs}$ \\
\hline 12 & 02_600_8CAB & $1,5 \mathrm{H}-\mathrm{brg}, 1,3 \mathrm{H} \delta$ & 52 & 02_1000_6CCA & $6 / 5 / 6 / 3 / 6 / 5 / 6 / 5 / 6 / 7 / 6 / 5 / 6 / 3$ \\
\hline 13 & 03_600_8CAB & $1,5 \mathrm{H}-\mathrm{brg}$ & 53 & 03_1000_6CCA & $2 \times(1,2 \mathrm{Me} \delta), 6 \rightarrow 3$ within $500 \mathrm{fs}$ \\
\hline 14 & $04 \_600 \_8 C A B$ & & 54 & 04_1000_6CCA & $2 \times(1,2 \mathrm{Me} \delta), 6 \rightarrow 3 \rightarrow 6(41 \mathrm{fs}) \rightarrow 3$ \\
\hline 15 & 05_600_8CAB & $1,5 \mathrm{H}-\mathrm{brg}$ & 55 & 05_1000_6CCA & $2 x(1,2 \mathrm{Me} \delta), 6 \rightarrow 3$ within $500 \mathrm{fs}$ \\
\hline 16 & $06 \_600 \_8 \mathrm{CAB}$ & $1,5 \mathrm{H}-\mathrm{brg}, 1,3 \mathrm{H} \delta$ & 56 & 06_1000_6CCA & $2 \times(1,2 \mathrm{Me} \delta), 6 \rightarrow 3$ within $500 \mathrm{fs}$ \\
\hline 17 & 07_600_8CAB & $1,3 \mathrm{H} \delta$ & 57 & 07_1000_6CCA & $2 \times(1,2 \mathrm{Me} \delta), 6 \rightarrow 3$ within $500 \mathrm{fs}$ \\
\hline 18 & 08_600_8CAB & $1,3 \mathrm{H} \delta$ & 58 & 08_1000_6CCA & $2 \times(1,2 \mathrm{Me} \delta), 6 \rightarrow 3$ within $500 \mathrm{fs}$ \\
\hline 19 & 09_600_8CAB & $1,3 \mathrm{H} \delta$ & 59 & 09_1000_6CCA & $2 \times(1,2 \mathrm{Me} \delta), 6 \rightarrow 3 \rightarrow 6(79 \mathrm{fs}) \rightarrow 3$ \\
\hline 20 & 10_600_8CAB & $1,3 \mathrm{H} \delta$ & 60 & 10_1000_6CCA & $2 x(1,2 \mathrm{Me} \delta), 6 \rightarrow 3$ within $500 \mathrm{fs}$ \\
\hline 21 & 01_1000_9CC & & 61 & 01_1600_1C & 1,2Me $\alpha, \mathrm{CH}_{4}$ elimination from 1 \\
\hline 22 & 02_1000_9CC & & 62 & 02_1600_1C & $1,2 \mathrm{Me} \alpha, 1 \mathrm{C} \rightarrow 3 \mathrm{C} \alpha, 3 \rightarrow 7 \mathrm{C} \rightarrow \beta \mathrm{sc}$ \\
\hline 23 & 03_1000_9CC & $9 C \rightarrow 6 C A \delta$ & 63 & 03_1600_1C & $1,2 \mathrm{Me \alpha}$ \\
\hline 24 & 04_1000_9CC & & 64 & 04_1600_1C & 1,2Mea \\
\hline 25 & 05_1000_9CC & & 65 & 05_1600_1C & \\
\hline 26 & 06_1000_9CC & $8 C A \rightarrow 5 C B \delta$ & 66 & 06_1600_1C & 1,2Me $\alpha$ \\
\hline 27 & 07_1000_9CC & & 67 & 07_1600_1C & 1,2Mea \\
\hline 28 & 08_1000_9CC & & 68 & 08_1600_1C & 1,2Me $\alpha$ \\
\hline 29 & 09_1000_9CC & & 69 & 09_1600_1C & 1,2Mea \\
\hline 30 & 10_1000_9CC & & 70 & 10_1600_1C & \\
\hline 31 & 01_1000_8CAB & & 71 & $01 \_1600 \_3 \mathrm{~N}$ & 1,2Me $\delta, 1,2 \mathrm{Et} \beta, 7 \mathrm{C} \rightarrow \beta \mathrm{sc}$ \\
\hline 32 & 02_1000_8CAB & & 72 & 02_1600_3N & 1,2Me $\delta, \mathrm{CH}_{4}$ elimination from 6 \\
\hline 33 & 03_1000_8CAB & & 73 & 03_1600_3N & $1,2 \mathrm{Me} \delta$ \\
\hline 34 & 04_1000_8CAB & $1,4 \mathrm{H}-\mathrm{brg}, 9 \mathrm{C} \rightarrow 6 \mathrm{CA} \delta$ & 74 & 04_1600_3N & $6 \mathrm{C} \rightarrow 9 \mathrm{C} \delta \rightarrow \beta s \mathrm{C}$ \\
\hline 35 & 05_1000_8CAB & $9 C \rightarrow 6 C A \delta$ & 75 & 05_1600_3N & $1,2 \mathrm{Me} \delta, 1,2 \mathrm{Et} \beta$ \\
\hline 36 & $06 \_1000 \_8 C A B$ & & 76 & 06_1600_3N & $1,2 \mathrm{H}$ to prim (40 fs), 1,2Me $\delta$ \\
\hline 37 & 07_1000_8CAB & & 77 & 07_1600_3N & $1,2 \mathrm{Me} \delta, 1,2 \mathrm{Et} \beta$ \\
\hline 38 & 08_1000_8CAB & & 78 & 08_1600_3N & $1,2 \mathrm{Me} \delta, \mathrm{C}_{2} \mathrm{H}_{6}$ elimination from 5 \\
\hline 39 & 09_1000_8CAB & & 79 & 09_1600_3N & $1,2 \mathrm{Me} \delta$ \\
\hline 40 & 10_1000_8CAB & & 80 & 10_1600_3N & $7 \rightarrow \beta s c$ \\
\hline
\end{tabular}

$\mathrm{brg}=$ bridge,$\beta s c=$ beta scission 


\section{Results from optimizations}

Seventy different transition states were found on the PBE/6-31G(d,p) potential energy surface (PES), and most were duplicated on the CCSD/6-31G(d,p) PES. We discuss the results grouped by step type: (i) twenty-eight 1,2-H shifts, (ii) nine 1,3-H shifts, (iii) eight 1,2-alkyl shifts, (iv) thirteen branching steps, and (v) twelve "triple-shift" carbon-scrambling steps.

1,2-H Shifts. Results appear in Figure S1. For the tert $\rightarrow \sec$ cases (upper plot), all examples combined the fundamental 1,2-H-shift between nonclinal structures with the fundamental internal rotation step from nonclinal secondary to closed-clinal secondary ions. Although conformer effects are noticeable (up to $4 \mathrm{kcal} \mathrm{mol}^{-1}$ for $\mathbf{2} \mathbf{7 5}$ ), presumably the ion most often follows the lowest-barrier path in each case (e.g. when doing $\mathbf{2} \rightarrow \mathbf{5 C A}$ or $\mathbf{3} \rightarrow \mathbf{6 C A}$ ). We took the $1^{\text {st }}$ of these 18 examples as being exemplary for tert $\rightarrow$ sec $1,2-\mathrm{H}$ shifts, and subject it to $\operatorname{CCSD}(\mathrm{T})$ single-point calculations for a more accurate estimate of the forward and reverse barrier heights (Section 3 below). The ethyl-closed form of $6(\mathbf{6 C B})$ is particularly prone to opening and performing this shift to a tertiary ion; only one conformer of $6 \mathrm{CB}$ could be found.

For the $\mathrm{sec} \rightarrow \mathrm{sec}$ cases (lower plot), these steps show more variation, connecting closed clinal, open nonclinal, or even open $\mathrm{H}$-bridged forms of secondary ions; the latter appeared on the CCSD potential energy surface but not the PBE one. The potential-energy-surface barriers are small, reaching 4-5 kcal mol${ }^{-1}$ only if the step starts with a closed form. Again we chose the $1^{\text {st }}$ example of this set as exemplary for higher-level calculation in Section 3. 

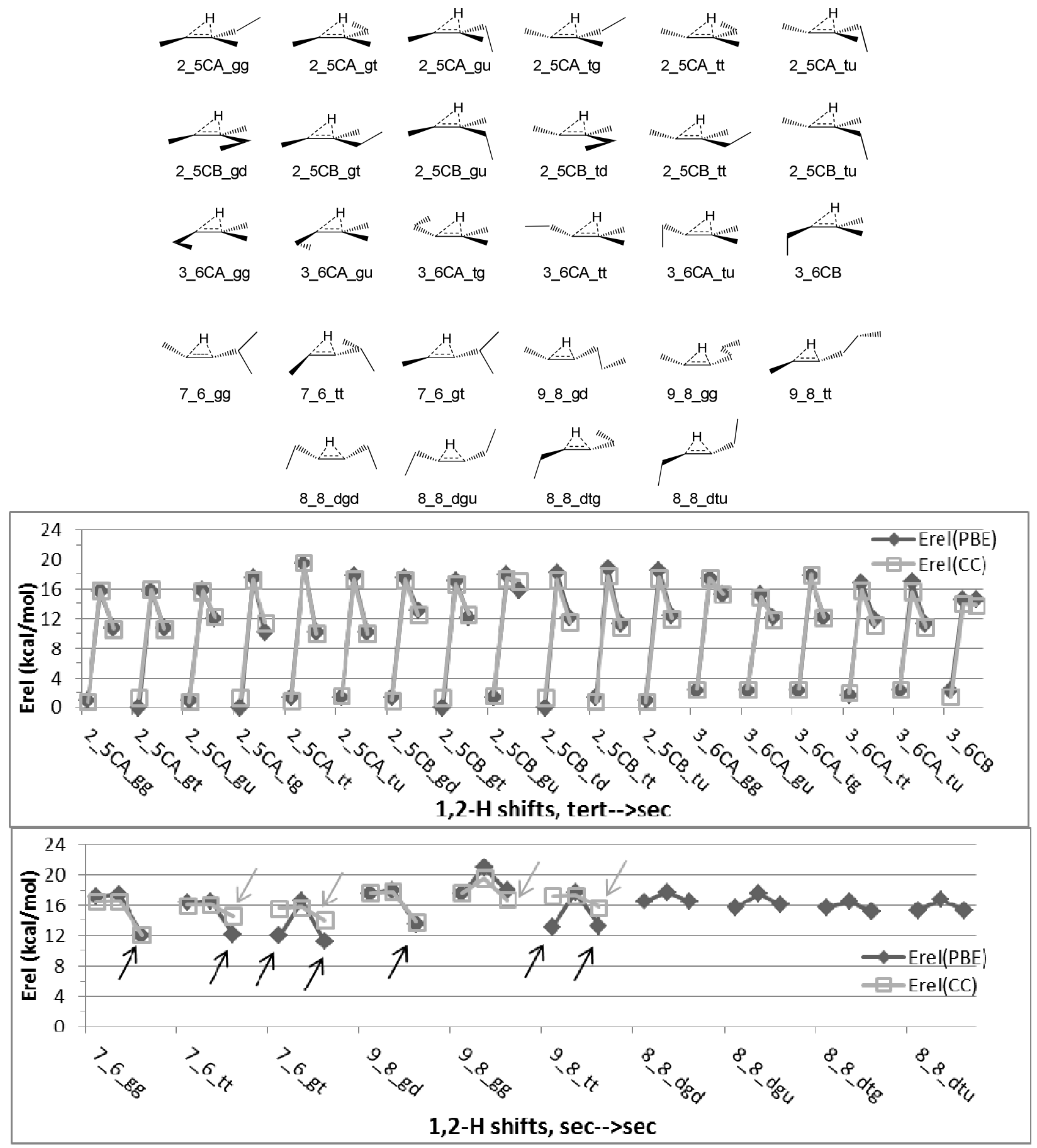

Figure S1: Results for 1,2-H-shifts in hexyl ions. Upper: the 28 transition state examples found, showing conformer variation. Middle and lower: the 28 reaction energy profiles . Data from PBE/6-31G(d,p) and CCSD/6-31G(d,p) optimizations; peaks are transition states. Energies are relative to global minimum. Upper plot: tert $\rightarrow$ sec cases $(6$ for $\mathbf{2 N} \rightarrow \mathbf{5 C A}, 6$ for $\mathbf{2 N} \rightarrow \mathbf{5 C B}, 5$ for $\mathbf{3 N} \rightarrow \mathbf{6 C A}, 1$ for $\mathbf{3 N} \rightarrow \mathbf{6 C B}$ ). Lower plot: $\sec \rightarrow \sec$ cases ( 3 for $\mathbf{7 N} \rightarrow \mathbf{6 N}, 3$ for $\mathbf{9 N} \rightarrow \mathbf{8 N}, 4$ for $\mathbf{8 N} \rightarrow \mathbf{8 N}$ ). In the lower plot, the intermediates are classical open nonclinal structures except for some $\mathrm{H}$-bridged (light arrows) and closed meso- $\mathrm{PCP}^{+}$

(dark arrows) cases. 
1,3-H and 1,2-Alkyl Shifts. Results appear in Figure S2, and only the $1^{\text {st }}$ of the 17 examples combines the fundamental step (within clinal $\mathrm{PCP}^{+}$structures) with an internal rotation step to a nonclinal structure $(\mathbf{7 C} \rightarrow \mathbf{3 C} \rightarrow \mathbf{3 N})$. For the $1,3-\mathrm{H}$ shifts (left plot in Figure $\mathrm{S} 2$ ), the lone tert $\rightarrow$ sec case $(3 \rightarrow 7$, in the $\alpha$-cycle in Figure 4$)$ has a very small reverse barrier; the eight sec $\rightarrow$ sec cases (within the $\beta$ and $\delta$ cycles) have barriers of $2-4 \mathrm{kcal} \mathrm{mol}^{-1}$ according to the PBE approximation. For the 1,2-alkyl shifts (right plot in Figure S2), the 8 cases are for the $\beta$-cycle 5CA $\rightarrow$ 5CA ethyl shift and the $\delta$-cycle $6 \mathrm{CA} \rightarrow \mathbf{5 C B}$ methyl shift, and the potential barriers are almost nonexistent $\left(<2 \mathrm{kcal} \mathrm{mol}^{-1}\right)$.

Saunders had misconcluded that 1,3-H-shifts would occur via different transition states than analogous "corner-to-corner" hydrogen shifts within protonated cyclopropanes; ${ }^{12}$ they are in fact the same step.

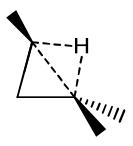

3

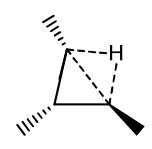

5CA duu

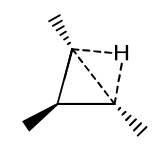

5CA udu

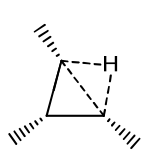

5CA uuu

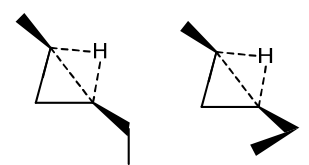

9C_8CA_gd 9C_8CA_gg

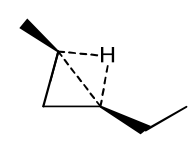

9C_8CA_gt

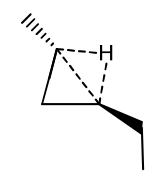

9C_8CA_td

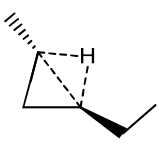

9C_8CA_tt
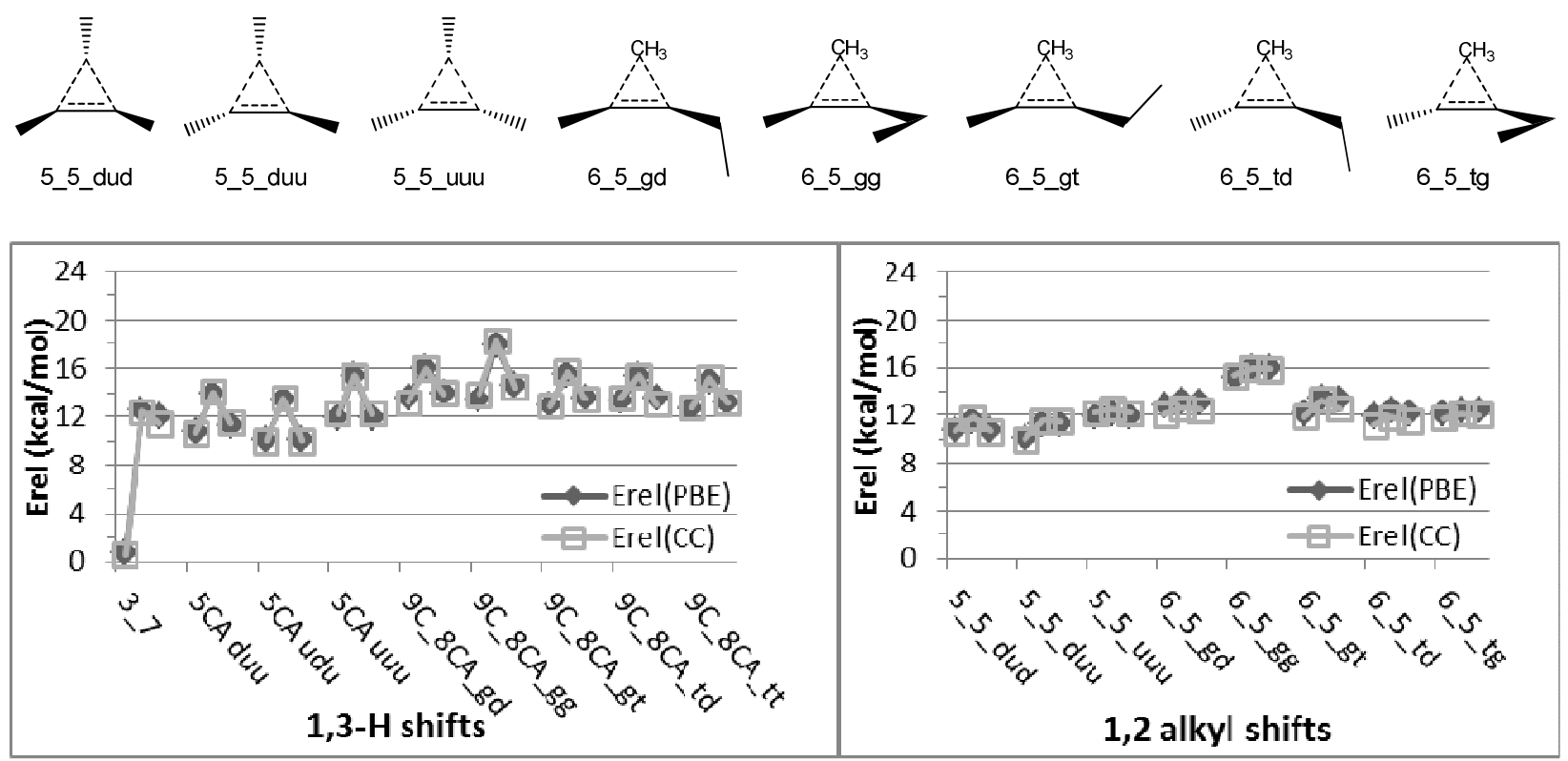

Figure S2: Results for 1,3-H-shifts (1 tert $\rightarrow \mathrm{sec}$ and $8 \mathrm{sec} \rightarrow \mathrm{sec}$ examples) and 1,2-alkyl shifts $8 \mathrm{sec} \rightarrow \mathrm{sec}$ examples) in hexyl ions. Data generated and plotted as in Figure S1. 
Branching Steps. Results appear in Figure S3 for 13 examples. The first 12 examples combine two fundamental steps (a 1,3-H shift and 1,2-R shift) into a single (elementary) step (Figure $\mathrm{S} 4$ ), with a meso- $\mathrm{PCP}^{+}$closed-primary carbocation structure as a transition state. The $13^{\text {th }}$ example combined three fundamental steps, because $4 \mathrm{C}$ was not a stationary state. All 13 examples occur in the $\delta$ and $\alpha \mathrm{PCP}^{+}$cycles (Figure 5). The closed-primary transition states lay 19-23 $\mathrm{kcal} \mathrm{mol}^{-1}$ above the global minimum on the potential energy surface. The $\mathbf{9 C} \rightarrow \mathbf{6 C A}$ and $\mathbf{8 C A} \rightarrow \mathbf{5 C B}$ examples are for sec $\rightarrow$ sec unbranched $\rightarrow$ monobranched steps, connecting meso$\mathrm{PCP}^{+}$minima with barriers of $8-10 \mathrm{kcal} \mathrm{mol}^{-1}$. The $\mathbf{7 C} \rightarrow \mathbf{1 C}$ and $\mathbf{3 C} \rightarrow \mathbf{1 C}$ steps are sec $\rightarrow$ tert and tert $\rightarrow$ tert (respectively) monobranched $\rightarrow$ dibranched steps, connecting open clinal structures which close to $\mathrm{PCP}^{+}$as part of the step. The Corma group in 1996 were the first to report finding the branching transition state computationally (for butyl and pentyl ions). ${ }^{30}$

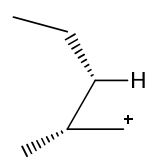

9_6_gd

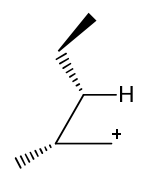

9_6_gt

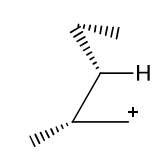

9_6_gu

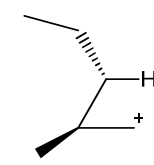

9_6_td

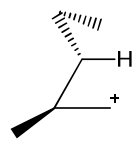

9_6_tg

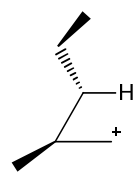

9_6_tt

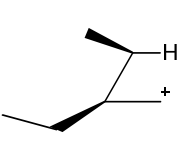

8_5_gd

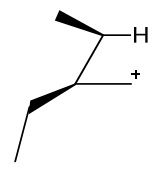

8_5_gt

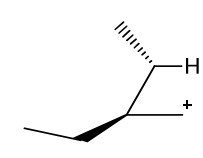

8_5_td

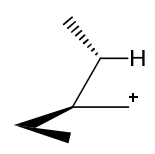

8_5_tg

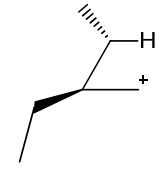

8_5_tt

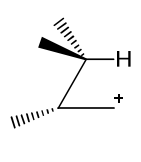

7_1_PH

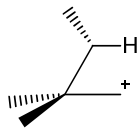

3_1_MHM

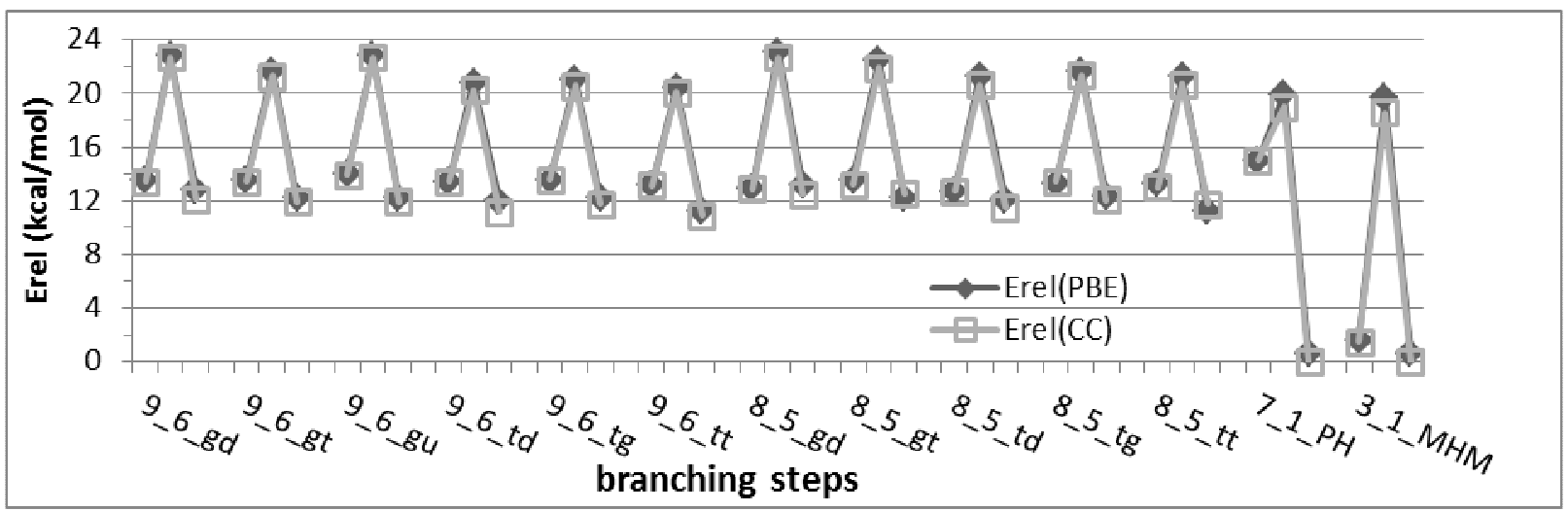

Figure S3: Results for $11 \mathrm{sec} \rightarrow \sec$ examples $(\mathbf{9 C} \rightarrow \mathbf{6 C A}$ and $\mathbf{8 C A} \rightarrow \mathbf{5 C B}, \delta$ cycle), and 1 sec $\rightarrow$ tert and 1 tert $\rightarrow$ tert example $(\mathbf{7 C} \rightarrow \mathbf{1 C}$ and $\mathbf{3 C} \rightarrow \mathbf{1 C}, \alpha$ cycle) of branching steps in hexyl ions. Data generated and plotted as in Fig. S1. 


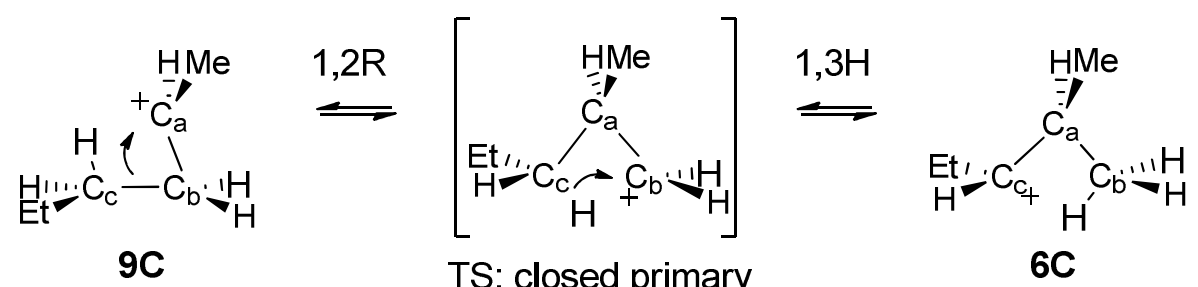

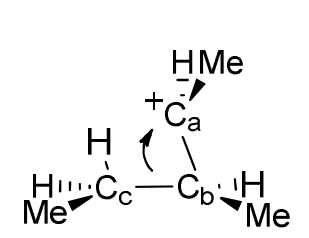

$8 \mathrm{C}$
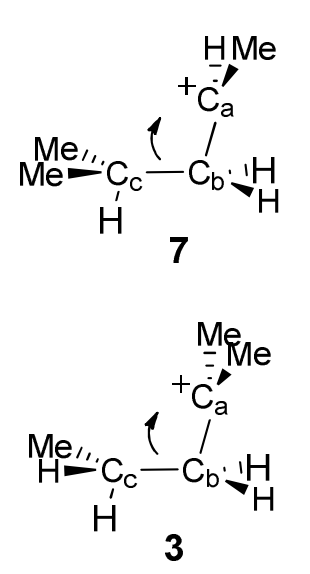

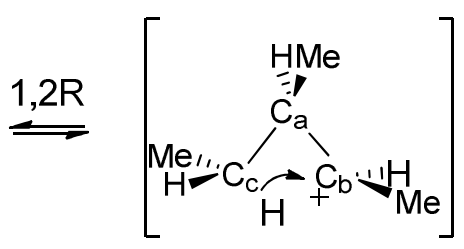

TS: closed primary

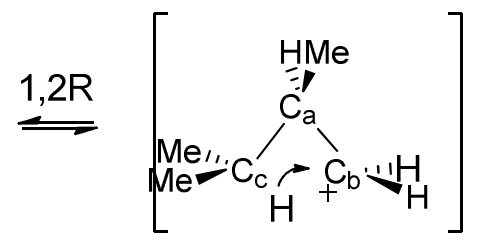

TS: closed primary

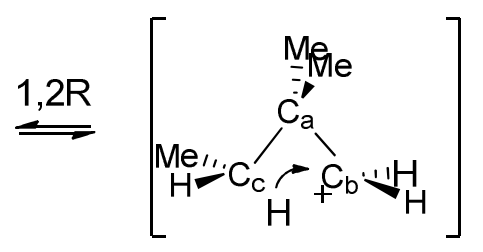

TS: closed primary

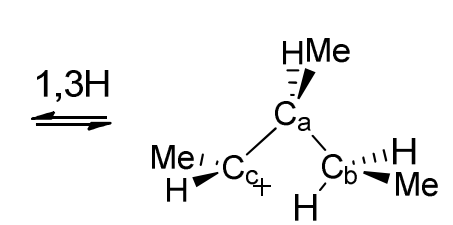

$5 \mathrm{C}$

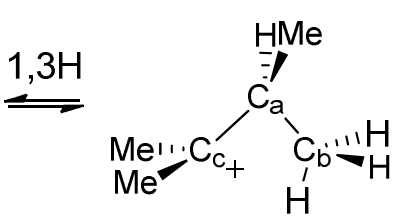

1

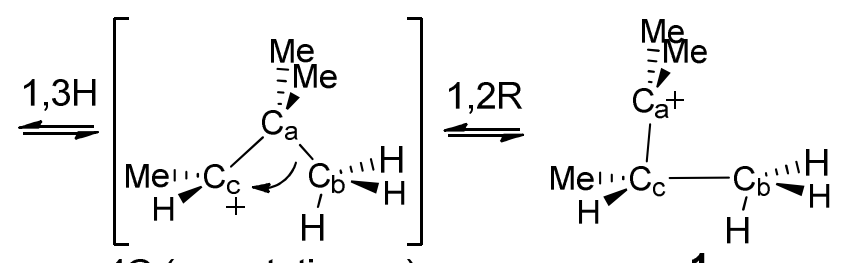

1

Figure S4: Branching steps. These appear in the $\alpha$ and $\delta$ cycles of Figure 5.

“Triple-Shift" Carbon-Scrambling Steps. Results appear in Figure S5. These steps feature 3 asynchronous shifts (Figure 6), with either an edge- or corner-protonated transition state lying between two meso-PCP ${ }^{+}$closed-primary structures in the $\gamma(\mathbf{2 C} \rightarrow \mathbf{2 C}), \varepsilon$ $(\mathbf{6 C B} \rightarrow \mathbf{6 C B})$, and $\zeta(\mathbf{8 C B} \rightarrow \mathbf{8 C B}) \mathrm{PCP}^{+}$cycles. In ${ }^{13} \mathrm{C}$-labelling experiments, these steps could alter the position of the labelled atom. As Figure 5 shows, each of these single-step transformations has two pathways: the "Me" path involves sequential 1,2-Me, 1,3-H, and 1,2-Me shifts (in the single step), while the "Bu" path involved sequential 1,3-H, 1,2-Bu, and 1,3-H shifts (in the single step). Each of the $\mathrm{Me}$ and $\mathrm{Bu}$ paths were available to more than one conformer, as well. The transition states lay $24-33 \mathrm{kcal} \mathrm{mol}^{-1}$ above the global minimum. The CCSD optimizations were not performed. 

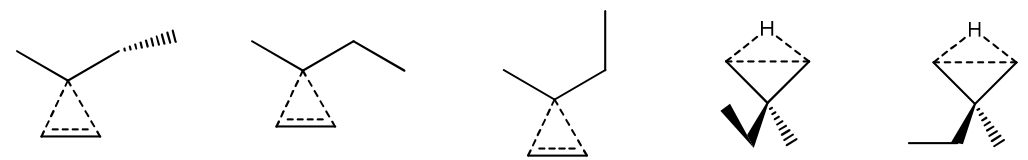

\section{2_2_Bu_gl}

2_2_Bu_gr

2_2_Bu_t

2_2_Me_g

2_2_Me_t
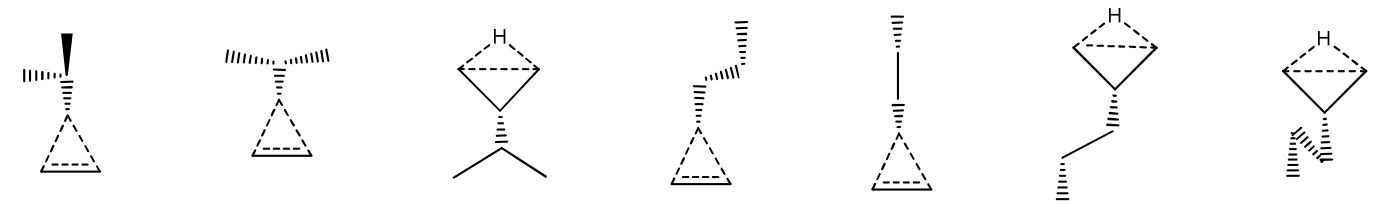

6_6_Bu_C1

6_6_Bu_Cs

6_6_Me_t

8_8_Bu_g

8_8_Bu_t

8_8_Me_g 8_8_Me_t

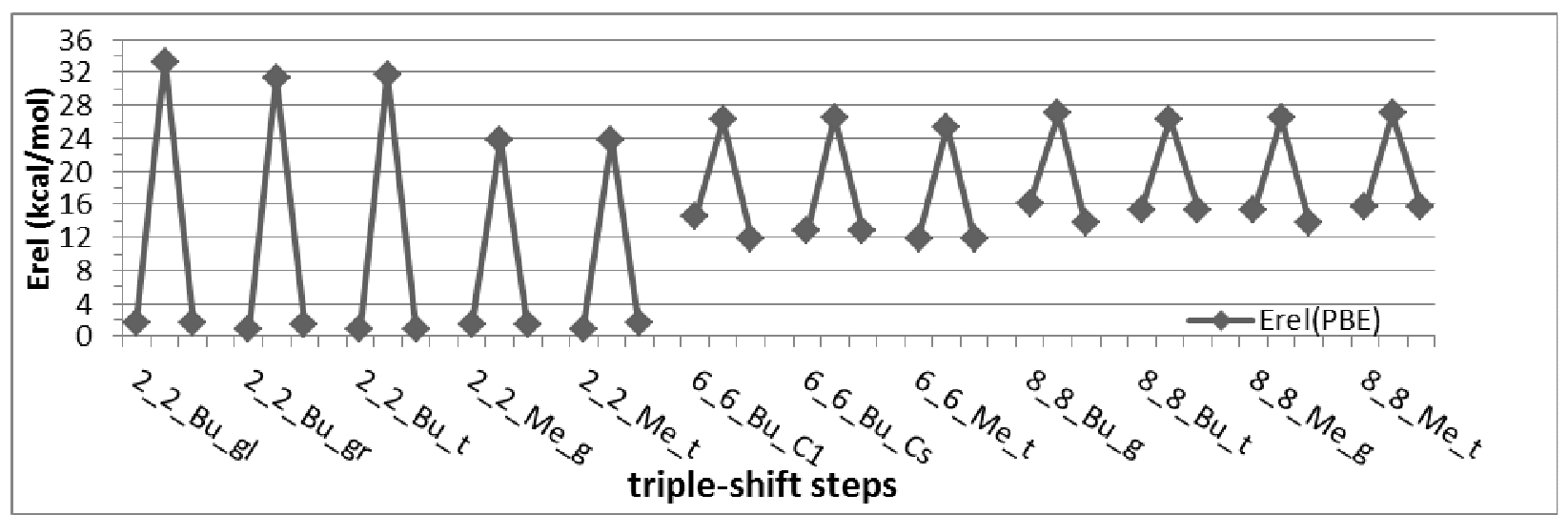

Figure S5: Results for 5 tert $\rightarrow$ tert $(\mathbf{2 C} \rightarrow \mathbf{2 C})$ and $7 \sec \rightarrow \sec (\mathbf{6 C B} \rightarrow \mathbf{6 C B}$ and $\mathbf{8 C B} \rightarrow \mathbf{8 C B})$ examples of "triple-shift" carbon-scrambling steps in hexyl ions. Data generated and plotted as in Fig. S1. 


\section{Computation of best barrier heights}

The values in Table 1 in the paper were generated as follows. First, high-accuracy single-point calculations [CCSD(T)/aug-cc-pVTZ//PBE/6-31G(d,p)] were performed for just 10 of the 70 elementary steps found, as well as the important $\beta$ scission step from 7C. Zero-point and thermochemistry corrections were computed at PBE/6-31G(d,p); vibrational frequencies were not scaled. The resulting logfile energies and corrections (in atomic units) were converted into barrier heights (in $\mathrm{kcal} \mathrm{mol}^{-1}$ ), Table S2. The original logfile energies appear in Table S3. The Table S2 barrier heights were modified to reduce the number of reaction types while achieving internal consistency, resulting in Table 1; the modifications are now discussed.

Table S2. Barrier heights $\left(\mathrm{kcal} \mathrm{mol}^{-1}\right)$, from CCSD(T)/aug-cc-pVTZ//PBE/6-31G(d,p) $\mathrm{E}_{\text {elec }}$ computation and rigid-rotor/harmonic-oscillator thermodynamic corrections from $\mathrm{PBE} / 6$ $31 \mathrm{G}(\mathrm{d}, \mathrm{p})$ frequency computation.

\begin{tabular}{|c|c|c|c|c|c|c|c|}
\hline Step type & Version & Direction & $\Delta^{\ddagger} \mathrm{E}_{\text {elec }}$ & $\Delta^{\ddagger} \mathrm{E}_{0}$ & $\Delta^{\ddagger} E_{298}$ & $\Delta^{\ddagger} H_{298}$ & $\Delta^{\ddagger} G_{298}$ \\
\hline \multirow{2}{*}{ t-s $1,2 \mathrm{H}$} & 2_5CA_gg & $1,2 \mathrm{H} 2 \mathrm{OC} \rightarrow 5 \mathrm{CCA}$ & 15.1 & 14.3 & 13.9 & 13.9 & 14.9 \\
\hline & & $1,2 \mathrm{H} 5 \mathrm{CCA} \rightarrow 2 \mathrm{OC}$ & 6.7 & 5.1 & 4.8 & 4.8 & 5.2 \\
\hline \multirow[t]{2}{*}{$\mathrm{s}-\mathrm{s} 1,2 \mathrm{H}$} & 7_6CA_gg & $1,2 \mathrm{H} 7 \mathrm{ON} \rightarrow 6 \mathrm{CA}$ & $-0.4^{b}$ & $-0.5^{\mathrm{b}}$ & $-0.9^{b}$ & $-0.9^{b}$ & $-0.1^{c}$ \\
\hline & & $1,2 \mathrm{H} 6 \mathrm{CA} \rightarrow 7 \mathrm{ON}$ & 5.0 & 3.6 & 3.2 & 3.2 & 4.2 \\
\hline \multirow[t]{2}{*}{ t-s $1,3 \mathrm{H}$} & 3_7C & $1,3 \mathrm{H} 3 \mathrm{OC} \rightarrow 7 \mathrm{CC}$ & 10.5 & 10.9 & 9.9 & 9.9 & $12.2^{\mathrm{a}}$ \\
\hline & & $1,3 \mathrm{H} 7 \mathrm{CC} \rightarrow 3 \mathrm{OC}$ & 1.0 & 0.6 & 0.1 & 0.1 & 1.3 \\
\hline \multirow[t]{2}{*}{ s-s $1,3 \mathrm{H}$} & 5CA_5CA_duu & $1,3 \mathrm{H} 5 \mathrm{CA} \rightarrow 5 \mathrm{CA}$ & 3.8 & 3.3 & 2.7 & 2.7 & 4.2 \\
\hline & & $1,3 \mathrm{H} 5 \mathrm{CA} \rightarrow 5 \mathrm{CA}$ & 3.5 & 3.0 & 2.4 & 2.4 & 4.0 \\
\hline \multirow[t]{2}{*}{ s-s $1,2 \mathrm{R}$} & 5CA_5CA_dud & $1,2 \mathrm{R} 5 \mathrm{CA} \rightarrow 5 \mathrm{CA}$ & 0.4 & 0.2 & -0.2 & -0.2 & 0.8 \\
\hline & & $1,2 \mathrm{R} 5 \mathrm{CA} \rightarrow 5 \mathrm{CA}$ & 0.4 & 0.2 & -0.2 & -0.2 & 0.8 \\
\hline \multirow[t]{2}{*}{ s-s branch } & 9C_6CA_tt & bra $9 \mathrm{C} \rightarrow 6 \mathrm{CA}$ & 6.5 & 6.0 & 5.6 & 5.6 & 6.8 \\
\hline & & bra $6 \mathrm{CA} \rightarrow 9 \mathrm{C}$ & 9.1 & 9.0 & 8.4 & 8.4 & 9.6 \\
\hline \multirow[t]{2}{*}{ s-t branch } & 1_7 & bra $70 C \rightarrow 10 C$ & $2.6^{\mathrm{b}}$ & $3.1^{\mathrm{b}}$ & $2.5^{b}$ & $2.5^{b}$ & $4.3^{c}$ \\
\hline & & bra $10 C \rightarrow 70 c$ & 17.6 & 18.3 & 17.6 & 17.6 & 19.4 \\
\hline \multirow[t]{2}{*}{ t-t branch } & 3_1 & bra $30 \mathrm{OC} \rightarrow 10 \mathrm{C}$ & 15.3 & 15.2 & 14.6 & 14.6 & 16.7 \\
\hline & & bra $10 \mathrm{C} \rightarrow 30 \mathrm{C}$ & 17.5 & 17.7 & 17.0 & 17.0 & 18.8 \\
\hline \multirow[t]{2}{*}{ t-t triple } & 2_2_Me_t & trip $20 \mathrm{OCOC} \rightarrow 2 \mathrm{OC}$ & 19.6 & 19.8 & 19.0 & 19.0 & 21.3 \\
\hline & & trip $20 C \rightarrow 2 O C O C$ & 20.0 & 19.5 & 18.6 & 18.6 & 21.0 \\
\hline \multirow[t]{2}{*}{ s-s triple } & 8CB_8CB_Me_t & trip $80 C B \rightarrow 80 C B$ & $9.9^{b}$ & $10.4^{b}$ & $9.6^{b}$ & $9.6^{b}$ & $11.6^{\mathrm{c}}$ \\
\hline & & trip $80 C B \rightarrow 80 C B$ & $9.9^{b}$ & $10.4^{b}$ & $9.6^{b}$ & $9.6^{b}$ & $11.6^{c}$ \\
\hline s-s $\beta$ scission & 7C $\beta$ scission & $7 \mathrm{OC} \rightarrow \mathrm{C}_{3} \mathrm{H}_{6} / \mathrm{C}_{3} \mathrm{H}_{7}^{+}$ & 36.7 & 31.7 & 32.2 & 32.8 & 19.1 \\
\hline
\end{tabular}

${ }^{\text {a }}$ Free energy correction for this $3 \mathrm{OC}$ conformer was poor due to a $6 \mathrm{~cm}^{-1}$ "harmonic" vibration; the correction was replaced by that of a different $30 \mathrm{C}$ conformer.

${ }^{\mathrm{b}}$ Computed value is too low an estimate: reactant energy/enthalpy is too high due to use of open structure. See page 12 for cure.

${ }^{c}$ Computed value is too low an estimate: reactant free energy is too high due to treating internal rotations as harmonic oscillators. See page 12 for cure. 
Table S3. CCSD(T)/aug-cc-pVTZ//PBE/6-31G(d,p) electronic energies (au) and PBE/6$31 \mathrm{G}(\mathrm{d}, \mathrm{p})$ thermochemical corrections (au) for Table 1.

\begin{tabular}{|c|c|c|c|c|c|}
\hline File & Eelectronic & $\delta \mathrm{E}_{0}$ & $\delta \mathrm{E}_{298}$ & $\delta \mathrm{H}_{298}$ & $\delta \mathrm{G}_{298}$ \\
\hline 2_5CA_gg_min1 (2OC) & -235.74389 & 0.17040 & 0.17925 & 0.18019 & 0.13742 \\
\hline 2_5CA_gg & -235.71990 & 0.16921 & 0.17737 & 0.17831 & 0.13713 \\
\hline 2_5CA_gg_min2 (5CCA) & -235.73050 & 0.17168 & 0.18026 & 0.18120 & 0.13947 \\
\hline 7_6CA_gg_min2 (7ON) & -235.71957 & 0.16887 & 0.17768 & 0.17863 & 0.13631 \\
\hline 7_6CA_gg & -235.72014 & 0.16862 & 0.17680 & 0.17774 & 0.13679 \\
\hline 7_6CA_gg_min1 (6OCA) & -235.72815 & 0.17092 & 0.17975 & 0.18070 & 0.13811 \\
\hline 3_7C_min1 (3OC) & -235.74404 & 0.17045 & 0.17954 & 0.18048 & $0.13771^{a}$ \\
\hline 3_7C & -235.72723 & 0.17097 & 0.17856 & 0.17951 & 0.14031 \\
\hline 3_7C_min2 (7CC) & -235.72876 & 0.17153 & 0.17993 & 0.18088 & 0.13981 \\
\hline 5CA_5CA_duu_min2 & -235.73051 & 0.17168 & 0.18026 & 0.18120 & 0.13947 \\
\hline 5CA_5CA_duu & -235.72442 & 0.17084 & 0.17853 & 0.17947 & 0.14000 \\
\hline 5CA_5CA_duu_min1 & -235.72994 & 0.17153 & 0.18021 & 0.18115 & 0.13919 \\
\hline 5CA_5CA_dud_min1 & -235.73051 & 0.17168 & 0.18026 & 0.18120 & 0.13947 \\
\hline 5CA_5CA_dud & -235.72989 & 0.17138 & 0.17936 & 0.18030 & 0.14018 \\
\hline 5CA_5CA_dud_min2 & -235.73051 & 0.17168 & 0.18026 & 0.18120 & 0.13947 \\
\hline 9C_6CA_tt_min1 (9C) & -235.72552 & 0.17206 & 0.18047 & 0.18142 & 0.13960 \\
\hline 9C_6CA_tt & -235.71511 & 0.17124 & 0.17892 & 0.17986 & 0.14004 \\
\hline 9C_6CA_tt_min2 (6CA) & -235.72958 & 0.17139 & 0.18003 & 0.18097 & 0.13922 \\
\hline 1_7_min1 (7OC) & -235.72192 & 0.17051 & 0.17931 & 0.18026 & 0.13796 \\
\hline $1 \_7$ & -235.71782 & 0.17143 & 0.17913 & 0.18007 & 0.14071 \\
\hline 1_7_min2 (1OC) & -235.74588 & 0.17027 & 0.17913 & 0.18007 & 0.13788 \\
\hline 3_1_min2 (3OC) & -235.74234 & 0.17066 & 0.17945 & 0.18040 & 0.13771 \\
\hline 3_1 & -235.71801 & 0.17064 & 0.17833 & 0.17928 & 0.14003 \\
\hline 3_1_min1 (1OC) & -235.74588 & 0.17027 & 0.17913 & 0.18007 & 0.13788 \\
\hline 2_2_Me_t_min1 (2OCOC) & -235.74389 & 0.17040 & 0.17925 & 0.18019 & 0.13739 \\
\hline 2_2_Me_t & -235.71269 & 0.17082 & 0.17831 & 0.17926 & 0.14015 \\
\hline 2_2_Me_t_min2 (2OC) & -235.74453 & 0.17161 & 0.18046 & 0.18140 & 0.13857 \\
\hline 8CB_8CB_Me_t_min1 (8OCB) & -235.72147 & 0.17102 & 0.17974 & 0.18069 & 0.13800 \\
\hline 8CB_8CB_Me_t & -235.70574 & 0.17186 & 0.17924 & 0.18018 & 0.14070 \\
\hline 8CB_8CB_Me_t_min2 (8OCB) & -235.72147 & 0.17102 & 0.17974 & 0.18069 & 0.13800 \\
\hline Beta scission: ispropyl & -117.98047 & 0.08584 & 0.09095 & 0.09190 & 0.05905 \\
\hline Beta scission: propene & -117.68988 & 0.07778 & 0.08193 & 0.08287 & 0.05274 \\
\hline
\end{tabular}

${ }^{\text {a }}$ This value taken from 3_1_min2 (3OC), since the original term was adversely affected by a small $6 \mathrm{~cm}^{-1}$ vibrational frequency, giving an erroneous entropy. 
Secondary carbocation energies. First we replaced open secondary carbocations with closed versions ( $7 \mathrm{ON}$ with $7 \mathrm{C}, 7 \mathrm{OC}$ with $7 \mathrm{C}, 8 \mathrm{OCB}$ with $8 \mathrm{CCA}$ ), to eliminate having to treat the very fast opening/closing of such ions as separate steps, and recalculated the relevant barriers of Table S2. This elimination of the opening step is justified by the extremely rapid timescales for interconverting between open and closed forms: in a 20 ps simulation of 8 (3-hexyl ion) at $298 \mathrm{~K}$ we saw frequent interconversion between closed clinal and open clinal forms, and at warmer temperatures we see open nonclinal forms participating on the same rapid timescale. A secondary carbocation is a dynamical species best represented by one energy value per constitutional isomer, if possible. We chose the closed form over the open form for the following reasons. On the potential energy surface, closed forms are clearly lower in energy than open forms (by 3-4 $\mathrm{kcal} \mathrm{mol}^{-1}$ normally). On the free energy surface, preference is for open forms, ${ }^{9 b}$ but the rigid-rotor/harmonic-oscillator estimate of the free energy of the open form is poor (internal rotation entropy), and this free energy is (somewhat paradoxically) better estimated by that of the closed form at $298 \mathrm{~K}$ ! (This is because there is only a slight preference for open forms in $298 \mathrm{~K}$ PBE simulations, and higher-accuracy corrections to electronic energy give slightly more benefit to closed forms, so we conclude that the open and closed forms of these ions have very similar free energies. The best estimate of this free energy would come from the calculation on the closed form, where the harmonic-oscillator approximation is better.)

Internal consistency. Next, we wanted to achieve internal consistency for the energy levels of the following classes of carbocations: unbranched secondary (8-9), monobranched secondary (5-7), monobranched tertiary (2-3), and dibranched tertiary (1). Our calculations predict nine unique energies for all hexyl ions 1-9, but this grouping appears to be legitimate to within 1 or $1.5 \mathrm{kcal} \mathrm{mol}^{-1}$ accuracy. To do this, we chose the data from the three branching reactions in Table S2, updated the barriers for "bra $7 \mathrm{OC} \rightarrow 10 \mathrm{OC}$ " according to the previous section, derived from these an anchoring reaction energy $\left(\Delta \mathrm{E}^{\mathrm{fwd}}\right.$ anchor $)$ for monobranched tert $\rightarrow$ monobranched sec (using $3 \rightarrow 7$ ), and corrected the monobranched tert $\rightarrow$ monobranched sec barrier heights for $1,2 \mathrm{H}$ and $1,3 \mathrm{H}$-shifts via

Forward from tertiary ion: $\quad \Delta \mathrm{E}_{\text {new }}^{\ddagger}=\Delta \mathrm{E}^{\ddagger}{ }_{\text {old }}+4 / 5\left(\Delta \mathrm{E}^{\mathrm{fwd}}{ }_{\text {anchor }}-\Delta \mathrm{E}^{\mathrm{fwd}}{ }_{\text {old }}\right)$

Reverse from secondary ion: $\Delta \mathrm{E}_{\text {new }}^{\ddagger}=\Delta \mathrm{E}^{\ddagger}{ }_{\text {old }}-1 / 5\left(\Delta \mathrm{E}^{\mathrm{fwd}}{ }_{\text {anchor }}-\Delta \mathrm{E}^{\mathrm{fwd}}{ }_{\text {old }}\right)$ 
which apply Evans/Polanyi shifts ${ }^{31}$ assuming the transition state is four-fifths of the way from tertiary to secondary regardless of the reaction route taken. These equations were applied for each type of energy (0K energies, $298 \mathrm{~K}$ enthalpies, etc.). For example, the corrected value for the $0 \mathrm{~K} \Delta \mathrm{E}^{\ddagger}$ for a tert $\rightarrow \sec 1,3 \mathrm{H}$-shift is $10.9+4 / 5(9.1-[10.9-0.6])=9.9=10 \mathrm{kcal} \mathrm{mol}^{-1}$. 


\section{Appendix. Transition-state Cartesian coordinates $(\AA)$, from CCSD/6-31G(d,p) (CC) and PBE/6-31G(d,p) (PBE) geometry optimizations.}

$\begin{array}{llll}\text { 1,2H } & & & \\ \text { 2_5CA_gg_CC } & & \\ \text { C } & 1.181762 & -0.714692 & 0.339225 \\ \text { C } & -0.007181 & 0.076575 & -0.308672 \\ \text { C } & -1.23102 & -0.689027 & -0.237076 \\ \text { C } & -2.557743 & -0.22722 & 0.130178 \\ \text { H } & 0.160691 & -0.047672 & -1.408415 \\ \text { H } & -1.136867 & -1.75052 & -0.466679 \\ \text { H } & -2.631951 & -0.581815 & 1.178723 \\ \text { H } & -2.684673 & 0.849637 & 0.126977 \\ \text { H } & -3.345221 & -0.757673 & -0.404747 \\ \text { H } & 1.052568 & -0.669081 & 1.422641 \\ \text { C } & -0.089649 & 1.555385 & 0.055594 \\ \text { H } & -0.874386 & 2.068264 & -0.498572 \\ \text { H } & -0.273391 & 1.676262 & 1.12374 \\ \text { H } & 0.850065 & 2.04603 & -0.181366 \\ \text { C } & 2.548644 & -0.161713 & -0.058867 \\ \text { H } & 2.676062 & -0.16766 & -1.141894 \\ \text { H } & 2.701802 & 0.851913 & 0.306081 \\ \text { H } & 3.325299 & -0.789114 & 0.375774 \\ \text { H } & 1.111122 & -1.764412 & 0.045437 \\ & & & \\ \text { 2_5CA_gg_PBE } & & \\ \text { C } & 1.214963 & -0.700777 & 0.369399 \\ \text { C } & -0.023525 & 0.080702 & -0.249495 \\ \text { C } & -1.237588 & -0.690919 & -0.20641 \\ \text { C } & -2.580831 & -0.235598 & 0.093921 \\ \text { H } & 0.160305 & -0.090638 & -1.359606 \\ \text { H } & -1.127436 & -1.764441 & -0.434371 \\ \text { H } & -2.833596 & -0.734134 & 1.064796 \\ \text { H } & -2.705286 & 0.84781 & 0.20715 \\ \text { H } & -3.326054 & -0.684346 & -0.591227 \\ \text { H } & 1.113303 & -0.59427 & 1.463434 \\ \text { C } & -0.089779 & 1.572727 & 0.059236 \\ \text { H } & -0.897132 & 2.078954 & -0.491188 \\ \text { H } & -0.243553 & 1.737306 & 1.138121 \\ \text { H } & 0.853647 & 2.060665 & -0.22009 \\ \text { H } & 1.114921 & -1.774661 & 0.135906 \\ \text { C } & 2.567463 & -0.17748 & -0.108669 \\ \text { H } & 2.733608 & 0.875499 & 0.162959 \\ \text { H } & 3.363589 & -0.765429 & 0.374746 \\ \text { H } & 2.68947 & -0.284247 & -1.198529\end{array}$




$\begin{array}{llll}\text { 2_5CA_gt_CC } & & \\ \text { C } & 1.356335 & 0.399824 & 0.385499 \\ \text { C } & 2.299012 & -0.717196 & -0.053634 \\ \text { C } & -0.042102 & 0.450348 & -0.344163 \\ \text { C } & -0.732125 & -0.814362 & -0.237485 \\ \text { C } & -2.101446 & -1.046138 & 0.186505 \\ \text { H } & 1.80413 & 1.371157 & 0.177871 \\ \text { H } & 1.963635 & -1.706112 & 0.260918 \\ \text { H } & 2.435897 & -0.722586 & -1.135959 \\ \text { H } & 3.275748 & -0.556622 & 0.399687 \\ \text { H } & 0.235914 & 0.413449 & -1.421394 \\ \text { H } & -0.14453 & -1.700892 & -0.469705 \\ \text { H } & -1.960158 & -1.3719 & 1.237402 \\ \text { H } & -2.735782 & -0.166656 & 0.184836 \\ \text { H } & -2.549906 & -1.903766 & -0.31504 \\ \text { H } & 1.166328 & 0.359902 & 1.459636 \\ \text { C } & -0.844334 & 1.707935 & -0.026679 \\ \text { H } & -1.748639 & 1.779732 & -0.629746 \\ \text { H } & -1.119216 & 1.73717 & 1.028058 \\ \text { H } & -0.235458 & 2.584651 & -0.236828 \\ & & & \\ \text { 2_5CA_gt_PBE } & & \\ & & & \\ \text { C } & 1.397058 & 0.413229 & 0.346225 \\ \text { C } & 2.303914 & -0.749952 & -0.03745 \\ \text { C } & -0.065602 & 0.454408 & -0.324573 \\ \text { C } & -0.74521 & -0.8069 & -0.216437 \\ \text { C } & -2.115897 & -1.049079 & 0.189157 \\ \text { H } & 1.84434 & 1.373339 & 0.045115 \\ \text { H } & 1.974285 & -1.716968 & 0.374561 \\ \text { H } & 2.417412 & -0.850624 & -1.129695 \\ \text { H } & 3.309897 & -0.561846 & 0.3699 \\ \text { H } & 0.234835 & 0.402575 & -1.410933 \\ \text { H } & -0.149256 & -1.69775 & -0.472013 \\ \text { H } & -2.066811 & -1.69604 & 1.098789 \\ \text { H } & -2.720856 & -0.157678 & 0.390761 \\ \text { H } & -2.61077 & -1.734155 & -0.531606 \\ \text { H } & 1.224219 & 0.454936 & 1.434422 \\ \text { C } & -0.837464 & 1.733415 & -0.016945 \\ \text { H } & -1.769024 & 1.806401 & -0.598788 \\ \text { H } & -1.087598 & 1.797196 & 1.054206 \\ \text { H } & -0.221479 & 2.609896 & -0.264576\end{array}$




$\begin{array}{llll}\text { 2_5CA_gu_CC } & & \\ \text { C } & -1.425006 & -0.117737 & 0.601141 \\ \text { C } & 0.014321 & 0.538552 & 0.58286 \\ \text { C } & 1.004072 & -0.514675 & 0.583983 \\ \text { C } & 2.100594 & -0.703357 & -0.347951 \\ \text { H } & 0.113102 & 0.92025 & 1.620265 \\ \text { H } & 0.87482 & -1.284075 & 1.345548 \\ \text { H } & 1.676393 & -1.453733 & -1.046417 \\ \text { H } & 2.364485 & 0.179583 & -0.920421 \\ \text { H } & 2.95823 & -1.193713 & 0.11188 \\ \text { C } & 0.191672 & 1.663046 & -0.43188 \\ \text { H } & 1.142018 & 2.177906 & -0.294618 \\ \text { H } & 0.140536 & 1.292593 & -1.454769 \\ \text { H } & -0.60399 & 2.393721 & -0.30022 \\ \text { H } & -1.495891 & -0.793928 & 1.455062 \\ \text { C } & -1.783744 & -0.841066 & -0.69488 \\ \text { H } & -2.804381 & -1.214522 & -0.628131 \\ \text { H } & -1.731482 & -0.179866 & -1.558489 \\ \text { H } & -1.133653 & -1.700566 & -0.87584 \\ \text { H } & -2.111635 & 0.70778 & 0.786517 \\ & & & \\ \text { 2_5CA_gu_PBE } & & \\ \text { H } & & & \\ \text { C } & 1.431026 & -0.211855 & -0.625844 \\ \text { C } & -0.033809 & 0.458906 & -0.573273 \\ \text { C } & -1.050766 & -0.555403 & -0.512343 \\ \text { C } & -2.181608 & -0.630042 & 0.391201 \\ \text { H } & -0.11608 & 0.748737 & -1.658778 \\ \text { H } & -0.947831 & -1.382006 & -1.234944 \\ \text { H } & -2.045661 & -1.565564 & 0.98764 \\ \text { H } & -2.312083 & 0.228052 & 1.060569 \\ \text { H } & -3.108862 & -0.867101 & -0.171659 \\ \text { C } & -0.152337 & 1.67611 & 0.338614 \\ \text { H } & -1.107916 & 2.202603 & 0.190803 \\ \text { H } & -0.071361 & 1.398464 & 1.400743 \\ \text { H } & 0.656497 & 2.388002 & 0.118575 \\ \text { H } & 1.434353 & -1.002589 & -1.394244 \\ \text { H } & 2.091245 & 0.594171 & -0.982818 \\ \text { C } & 1.891554 & -0.748542 & 0.727554 \\ \text { H } & 2.925522 & -1.115588 & 0.622116 \\ \text { H } & 1.904414 & 0.028201 & 1.506857 \\ & 1.273395 & -1.59042 & 1.079679\end{array}$




$\begin{array}{llll}\text { 2_5CA_tg_CC } & & \\ \text { C } & 0.73877 & -0.821727 & 0.347979 \\ \mathrm{C} & -0.020505 & 0.422201 & -0.233308 \\ \mathrm{C} & -1.444497 & 0.389125 & 0.039049 \\ \mathrm{C} & -2.318777 & -0.767488 & -0.079573 \\ \mathrm{H} & -0.053496 & 0.240583 & -1.3331 \\ \mathrm{H} & -1.925755 & 1.313562 & 0.363448 \\ \mathrm{H} & -3.29619 & -0.481162 & -0.472094 \\ \mathrm{H} & -1.88913 & -1.616041 & -0.600943 \\ \mathrm{H} & -2.511767 & -1.051295 & 0.972253 \\ \mathrm{H} & 0.713176 & -0.738685 & 1.436675 \\ \mathrm{C} & 0.64128 & 1.769425 & 0.061224 \\ \mathrm{H} & 0.031883 & 2.597568 & -0.300167 \\ \mathrm{H} & 0.796994 & 1.889376 & 1.133657 \\ \mathrm{H} & 1.608021 & 1.831984 & -0.430639 \\ \mathrm{C} & 2.182435 & -0.899642 & -0.144598 \\ \mathrm{H} & 2.227072 & -0.932847 & -1.233665 \\ \mathrm{H} & 2.778416 & -0.059064 & 0.204723 \\ \mathrm{H} & 2.638282 & -1.810657 & 0.240397 \\ \mathrm{H} & 0.210257 & -1.734687 & 0.074813 \\ & & & \\ \mathrm{H} \text {-5CA_tg_PBE } & & \\ & & & \\ \mathrm{C} & 0.782816 & -0.810432 & 0.388322 \\ \mathrm{C} & -0.034703 & 0.430746 & -0.188163 \\ \mathrm{C} & -1.458204 & 0.369696 & 0.041608 \\ \mathrm{C} & -2.320547 & -0.790663 & -0.092201 \\ \mathrm{H} & -0.049726 & 0.184397 & -1.293207 \\ \mathrm{H} & -1.966306 & 1.305814 & 0.333448 \\ \mathrm{H} & -3.242929 & -0.522251 & -0.646221 \\ \mathrm{H} & -1.852428 & -1.701417 & -0.485189 \\ \mathrm{H} & -2.723308 & -0.990377 & 0.931468 \\ \mathrm{H} & 0.803229 & -0.664257 & 1.482119 \\ \mathrm{C} & 0.619158 & 1.795024 & 0.044056 \\ \mathrm{H} & -0.01673 & 2.618778 & -0.314502 \\ \mathrm{H} & 0.817529 & 1.948409 & 1.11696 \\ \mathrm{H} & 1.579164 & 1.861742 & -0.485368 \\ \mathrm{C} & 2.197716 & -0.903781 & -0.176758 \\ \mathrm{H} & 2.199433 & -1.052382 & -1.268415 \\ \mathrm{H} & 2.80567 & -0.018281 & 0.059561 \\ \mathrm{H} & 2.699299 & -1.772331 & 0.27967 \\ & 0.229692 & -1.741387 & 0.188483\end{array}$




$\begin{array}{llll}\text { 2_5CA_tt_CC } & & \\ \text { C } & -0.187402 & -1.257961 & 0.302395 \\ \mathrm{C} & -1.64936 & -1.370127 & -0.127876 \\ \mathrm{C} & 0.673553 & -0.069954 & -0.239149 \\ \mathrm{C} & 0.249304 & 1.285245 & 0.048732 \\ \mathrm{C} & -1.096929 & 1.83982 & 0.026373 \\ \mathrm{H} & 0.352107 & -2.142669 & -0.035027 \\ \mathrm{H} & -2.298429 & -0.664123 & 0.386393 \\ \mathrm{H} & -1.765978 & -1.241984 & -1.205154 \\ \mathrm{H} & -2.008939 & -2.366964 & 0.122597 \\ \mathrm{H} & 0.463468 & -0.020373 & -1.339262 \\ \mathrm{H} & 1.038467 & 2.003931 & 0.277535 \\ \mathrm{H} & -1.090955 & 2.867632 & -0.338006 \\ \mathrm{H} & -1.840304 & 1.225315 & -0.464 \\ \mathrm{H} & -1.350454 & 1.918384 & 1.10121 \\ \mathrm{H} & -0.102873 & -1.2419 & 1.391195 \\ \mathrm{C} & 2.172102 & -0.299602 & -0.014503 \\ \mathrm{H} & 2.77107 & 0.520835 & -0.40895 \\ \mathrm{H} & 2.381121 & -0.408533 & 1.049726 \\ \mathrm{H} & 2.484093 & -1.214078 & -0.514094 \\ & & & \\ \mathrm{H} \text {-5CA_tt_PBE } & & \\ & & & \\ \mathrm{C} & -0.214188 & -1.291739 & 0.260719 \\ \mathrm{C} & -1.696297 & -1.349761 & -0.102665 \\ \mathrm{C} & 0.678454 & -0.069042 & -0.207165 \\ \mathrm{C} & 0.271238 & 1.286724 & 0.075869 \\ \mathrm{C} & -1.051505 & 1.884008 & 0.012756 \\ \mathrm{H} & 0.305386 & -2.162665 & -0.168942 \\ \mathrm{H} & -2.316101 & -0.648923 & 0.476182 \\ \mathrm{H} & -1.87508 & -1.188889 & -1.17856 \\ \mathrm{H} & -2.070645 & -2.358536 & 0.133501 \\ \mathrm{H} & 0.437287 & -0.002666 & -1.317742 \\ \mathrm{H} & 1.083142 & 1.994421 & 0.321405 \\ \mathrm{H} & -1.007226 & 2.839547 & -0.549323 \\ \mathrm{H} & -1.864689 & 1.240538 & -0.334759 \\ \mathrm{H} & -1.264688 & 2.248245 & 1.048028 \\ \mathrm{H} & -0.072619 & -1.347316 & 1.354173 \\ \mathrm{C} & 2.175154 & -0.347488 & -0.019588 \\ \mathrm{H} & 2.800831 & 0.492021 & -0.358694 \\ \mathrm{H} & 2.395817 & -0.540603 & 1.042244 \\ & 2.471446 & -1.241377 & -0.587063\end{array}$




\begin{tabular}{|c|c|c|c|}
\hline \multicolumn{4}{|c|}{ 2_5CA_tu_CC } \\
\hline $\mathrm{C}$ & -0.784331 & -0.750476 & 0.713109 \\
\hline $\mathrm{C}$ & -0.098159 & 0.63119 & 0.400106 \\
\hline C & 1.147311 & 0.481871 & -0.325865 \\
\hline $\mathrm{H}$ & 0.293159 & 0.952703 & 1.390015 \\
\hline $\mathrm{C}$ & -1.041273 & 1.69137 & -0.169671 \\
\hline $\mathrm{H}$ & -0.547919 & 2.660323 & -0.239398 \\
\hline $\mathrm{H}$ & -1.396336 & 1.410957 & -1.160159 \\
\hline $\mathrm{H}$ & -1.906447 & 1.801394 & 0.481258 \\
\hline $\mathrm{H}$ & -0.086742 & -1.393198 & 1.249983 \\
\hline $\mathrm{C}$ & -1.320985 & -1.455809 & -0.531848 \\
\hline $\mathrm{H}$ & -1.808042 & -2.383958 & -0.237063 \\
\hline $\mathrm{H}$ & -2.056943 & -0.849586 & -1.056932 \\
\hline $\mathrm{H}$ & -0.521732 & -1.711258 & -1.231273 \\
\hline $\mathrm{H}$ & -1.595469 & -0.518605 & 1.402782 \\
\hline $\mathrm{C}$ & 2.225976 & -0.436986 & 0.00552 \\
\hline $\mathrm{H}$ & 2.236773 & -1.167495 & -0.822093 \\
\hline $\mathrm{H}$ & 3.190235 & 0.072763 & -0.076813 \\
\hline $\mathrm{H}$ & 2.111656 & -0.954028 & 0.951902 \\
\hline $\mathrm{H}$ & 1.316567 & 1.113031 & -1.200315 \\
\hline \multicolumn{4}{|c|}{ 2_5CA_tu_PBE } \\
\hline $\mathrm{C}$ & -0.83084 & -0.746489 & 0.696975 \\
\hline $\mathrm{C}$ & -0.037747 & 0.609717 & 0.373577 \\
\hline $\mathrm{C}$ & 1.207934 & 0.413772 & -0.325969 \\
\hline $\mathrm{H}$ & 0.357806 & 0.833678 & 1.405283 \\
\hline $\mathrm{C}$ & -0.919349 & 1.754281 & -0.13192 \\
\hline $\mathrm{H}$ & -0.367759 & 2.706291 & -0.153921 \\
\hline $\mathrm{H}$ & -1.296315 & 1.551088 & -1.145598 \\
\hline $\mathrm{H}$ & -1.787998 & 1.884229 & 0.529693 \\
\hline $\mathrm{H}$ & -0.142698 & -1.472412 & 1.157685 \\
\hline $\mathrm{C}$ & -1.519904 & -1.336861 & -0.533018 \\
\hline $\mathrm{H}$ & -2.091423 & -2.22464 & -0.21634 \\
\hline $\mathrm{H}$ & -2.236211 & -0.63821 & -0.99012 \\
\hline $\mathrm{H}$ & -0.801443 & -1.658034 & -1.304494 \\
\hline $\mathrm{H}$ & -1.565837 & -0.464974 & 1.467257 \\
\hline $\mathrm{C}$ & 2.241537 & -0.555092 & -0.00769 \\
\hline $\mathrm{H}$ & 2.358987 & -1.208491 & -0.90517 \\
\hline $\mathrm{H}$ & 3.229597 & -0.048875 & 0.024265 \\
\hline $\mathrm{H}$ & 2.070664 & -1.167857 & 0.88582 \\
\hline $\mathrm{H}$ & 1.422842 & 1.072238 & -1.186083 \\
\hline
\end{tabular}




\begin{tabular}{|c|c|c|c|}
\hline \multicolumn{4}{|c|}{ 2_5CB_gd_CC } \\
\hline C & -1.346284 & -1.672732 & 0.074827 \\
\hline C & -0.354487 & -0.517171 & -0.238063 \\
\hline $\mathrm{C}$ & -1.022794 & 0.761888 & -0.303145 \\
\hline $\mathrm{C}$ & -0.587426 & 2.047237 & 0.217867 \\
\hline $\mathrm{H}$ & -0.866766 & -2.615682 & -0.173514 \\
\hline $\mathrm{H}$ & -0.180913 & -0.594001 & -1.345391 \\
\hline $\mathrm{H}$ & -1.990089 & 0.760356 & -0.806428 \\
\hline $\mathrm{H}$ & -1.314416 & 2.237421 & 1.029972 \\
\hline $\mathrm{H}$ & 0.418157 & 2.067675 & 0.618022 \\
\hline $\mathrm{H}$ & -0.775417 & 2.84931 & -0.500193 \\
\hline $\mathrm{H}$ & -1.579907 & -1.66843 & 1.138348 \\
\hline $\mathrm{C}$ & 0.98614 & -0.594169 & 0.504123 \\
\hline $\mathrm{H}$ & 0.888799 & -0.102425 & 1.475424 \\
\hline $\mathrm{H}$ & 1.175108 & -1.645841 & 0.720085 \\
\hline $\mathrm{C}$ & 2.168474 & -0.028903 & -0.287531 \\
\hline $\mathrm{H}$ & 2.315092 & -0.591345 & -1.210533 \\
\hline $\mathrm{H}$ & 2.03509 & 1.02031 & -0.554576 \\
\hline $\mathrm{H}$ & 3.083053 & -0.106162 & 0.297349 \\
\hline $\mathrm{H}$ & -2.269529 & -1.588086 & -0.497034 \\
\hline \multicolumn{4}{|c|}{ 2_5CB_gd_PBE } \\
\hline $\mathrm{C}$ & -1.376673 & -1.663281 & 0.083604 \\
\hline C & -0.34933 & -0.511736 & -0.237413 \\
\hline $\mathrm{C}$ & -1.008487 & 0.768667 & -0.320188 \\
\hline $\mathrm{C}$ & -0.594702 & 2.039143 & 0.239183 \\
\hline $\mathrm{H}$ & -0.910311 & -2.621803 & -0.179037 \\
\hline $\mathrm{H}$ & -0.196057 & -0.660852 & -1.352449 \\
\hline $\mathrm{H}$ & -1.959877 & 0.777079 & -0.87858 \\
\hline $\mathrm{H}$ & -1.429995 & 2.366723 & 0.905437 \\
\hline $\mathrm{H}$ & 0.356324 & 2.040762 & 0.782416 \\
\hline $\mathrm{H}$ & -0.62987 & 2.829784 & -0.541065 \\
\hline $\mathrm{H}$ & -1.590141 & -1.6474 & 1.162583 \\
\hline $\mathrm{C}$ & 0.997098 & -0.617409 & 0.494035 \\
\hline $\mathrm{H}$ & 0.904223 & -0.153251 & 1.492527 \\
\hline $\mathrm{H}$ & 1.185845 & -1.686855 & 0.681578 \\
\hline $\mathrm{C}$ & 2.181219 & -0.024188 & -0.283837 \\
\hline $\mathrm{H}$ & 2.326857 & -0.543144 & -1.245659 \\
\hline $\mathrm{H}$ & 2.058209 & 1.049925 & -0.500851 \\
\hline $\mathrm{H}$ & 3.109931 & -0.137173 & 0.294232 \\
\hline $\mathrm{H}$ & -2.319893 & -1.560975 & -0.47343 \\
\hline
\end{tabular}




$\begin{array}{llll}\text { 2_5CB_gt_CC } & & \\ \text { C } & -0.41479 & 1.739122 & 0.291757 \\ \text { C } & 0.001522 & 0.373937 & -0.347909 \\ \text { C } & 1.44795 & 0.271073 & -0.333179 \\ \text { C } & 2.260504 & -0.815977 & 0.177573 \\ \text { H } & -1.457616 & 1.924232 & 0.053771 \\ \text { H } & -0.165109 & 0.526564 & -1.438105 \\ \text { H } & 1.987162 & 1.140168 & -0.710719 \\ \text { H } & 2.538795 & -0.423962 & 1.178564 \\ \text { H } & 1.73506 & -1.754157 & 0.317384 \\ \text { H } & 3.204128 & -0.919442 & -0.357434 \\ \text { H } & -0.302054 & 1.685587 & 1.373583 \\ \text { C } & -0.783923 & -0.843028 & 0.150397 \\ \text { H } & -0.426529 & -1.73403 & -0.37098 \\ \text { H } & -0.575221 & -0.987093 & 1.214153 \\ \text { C } & -2.292461 & -0.715081 & -0.070177 \\ \text { H } & -2.729554 & 0.069645 & 0.545006 \\ \text { H } & -2.519677 & -0.503723 & -1.116245 \\ \text { H } & -2.78111 & -1.650884 & 0.194112 \\ \text { H } & 0.178907 & 2.56682 & -0.093858 \\ & & & \\ \text { 2_5CB_gt_PBE } & & \\ \text { H } & & & \\ \text { C } & -0.402029 & 1.771616 & 0.284727 \\ \text { C } & 0.006267 & 0.360132 & -0.308434 \\ \text { C } & 1.445541 & 0.256013 & -0.329868 \\ \text { C } & 2.274766 & -0.820972 & 0.167358 \\ \text { H } & -1.462328 & 1.934872 & 0.053128 \\ \text { H } & -0.190429 & 0.527761 & -1.408244 \\ \text { H } & 1.980048 & 1.114193 & -0.770957 \\ \text { H } & 2.920143 & -0.371852 & 0.962587 \\ \text { H } & 1.744328 & -1.696247 & 0.558359 \\ \text { H } & 3.04075 & -1.093832 & -0.589846 \\ \text { H } & -0.262321 & 1.752614 & 1.375183 \\ \text { C } & -0.812151 & -0.829679 & 0.211498 \\ \text { H } & -0.411808 & -1.759672 & -0.229573 \\ \text { H } & -0.666479 & -0.908351 & 1.304266 \\ \text { C } & -2.308737 & -0.728291 & -0.112801 \\ \text { H } & -2.795371 & 0.109774 & 0.40912 \\ \text { H } & -2.48108 & -0.610264 & -1.195586 \\ \text { H } & -2.821687 & -1.64846 & 0.202808 \\ & 0.184289 & 2.596546 & -0.146127\end{array}$




\begin{tabular}{|c|c|c|c|}
\hline \multicolumn{4}{|c|}{ 2_5CB_gu_CC } \\
\hline C & 0.47836 & 1.717392 & -0.496207 \\
\hline C & 0.007098 & 0.638291 & 0.56724 \\
\hline C & -1.342466 & 0.328761 & 0.153013 \\
\hline C & -1.897656 & -0.945872 & -0.27104 \\
\hline $\mathrm{H}$ & 1.487329 & 1.996739 & -0.199015 \\
\hline $\mathrm{H}$ & -0.105928 & 1.234132 & 1.486167 \\
\hline $\mathrm{H}$ & -2.041288 & 1.165371 & 0.165467 \\
\hline $\mathrm{H}$ & -2.514318 & -0.818543 & -1.16588 \\
\hline $\mathrm{H}$ & -1.181586 & -1.751826 & -0.366877 \\
\hline $\mathrm{H}$ & -2.630283 & -1.185129 & 0.520942 \\
\hline $\mathrm{H}$ & 0.506599 & 1.303112 & -1.500749 \\
\hline $\mathrm{C}$ & 0.993922 & -0.518817 & 0.769598 \\
\hline $\mathrm{H}$ & 1.800393 & -0.135361 & 1.396961 \\
\hline $\mathrm{H}$ & 0.506702 & -1.300799 & 1.357205 \\
\hline $\mathrm{C}$ & 1.598904 & -1.106096 & -0.50965 \\
\hline $\mathrm{H}$ & 0.844628 & -1.446249 & -1.220629 \\
\hline $\mathrm{H}$ & 2.233509 & -0.382263 & -1.018674 \\
\hline $\mathrm{H}$ & 2.219802 & -1.963252 & -0.255548 \\
\hline $\mathrm{H}$ & -0.154531 & 2.602113 & -0.477094 \\
\hline \multicolumn{4}{|c|}{ 2_5CB_gu_PBE } \\
\hline $\mathrm{C}$ & 0.390945 & 1.743369 & -0.512548 \\
\hline $\mathrm{C}$ & -0.013995 & 0.62506 & 0.571325 \\
\hline $\mathrm{C}$ & -1.357705 & 0.278983 & 0.182322 \\
\hline C & -1.871314 & -1.000474 & -0.26418 \\
\hline $\mathrm{H}$ & 1.394493 & 2.074223 & -0.202753 \\
\hline $\mathrm{H}$ & -0.122574 & 1.254643 & 1.485338 \\
\hline $\mathrm{H}$ & -2.089963 & 1.103052 & 0.214755 \\
\hline $\mathrm{H}$ & -2.405936 & -0.873763 & -1.231089 \\
\hline $\mathrm{H}$ & -1.149445 & -1.823171 & -0.301717 \\
\hline $\mathrm{H}$ & -2.723011 & -1.251784 & 0.412344 \\
\hline $\mathrm{H}$ & 0.442003 & 1.315584 & -1.521818 \\
\hline $\mathrm{C}$ & 1.037874 & -0.478486 & 0.767422 \\
\hline $\mathrm{H}$ & 1.84396 & -0.04018 & 1.380607 \\
\hline $\mathrm{H}$ & 0.59589 & -1.278226 & 1.387759 \\
\hline $\mathrm{C}$ & 1.652179 & -1.067811 & -0.512139 \\
\hline $\mathrm{H}$ & 0.90261 & -1.507572 & -1.190744 \\
\hline $\mathrm{H}$ & 2.216882 & -0.313775 & -1.081426 \\
\hline $\mathrm{H}$ & 2.357719 & -1.868232 & -0.245161 \\
\hline $\mathrm{H}$ & -0.290531 & 2.605357 & -0.499309 \\
\hline
\end{tabular}




$\begin{array}{llll}\text { 2_5CB_td_CC } & & \\ \text { C } & 0.754128 & 1.735425 & -0.011625 \\ \mathrm{C} & 0.001712 & 0.386181 & -0.226103 \\ \mathrm{C} & 0.784111 & -0.753509 & 0.220306 \\ \mathrm{C} & 2.189537 & -0.989397 & -0.06648 \\ \mathrm{H} & 0.170577 & 2.515855 & -0.493056 \\ \mathrm{H} & 0.015966 & 0.215695 & -1.325586 \\ \mathrm{H} & 0.294922 & -1.508102 & 0.837486 \\ \mathrm{H} & 2.372697 & -2.048698 & -0.260548 \\ \mathrm{H} & 2.617045 & -0.343509 & -0.825868 \\ \mathrm{H} & 2.689991 & -0.798209 & 0.901136 \\ \mathrm{H} & 0.817035 & 1.953358 & 1.053416 \\ \mathrm{C} & -1.447132 & 0.400257 & 0.27989 \\ \mathrm{H} & -1.436988 & 0.476752 & 1.370525 \\ \mathrm{H} & -1.911827 & 1.315991 & -0.086863 \\ \mathrm{C} & -2.268361 & -0.807124 & -0.173452 \\ \mathrm{H} & -2.31108 & -0.865085 & -1.26196 \\ \mathrm{H} & -1.866286 & -1.751582 & 0.201343 \\ \mathrm{H} & -3.288479 & -0.727848 & 0.197021 \\ \mathrm{H} & 1.75246 & 1.734384 & -0.44226 \\ & & & \\ \mathrm{H} \text {-5CB_td_PBE } & & \\ & & & \\ \mathrm{C} & 0.742685 & 1.769763 & -0.021107 \\ \mathrm{C} & 0.000794 & 0.385199 & -0.2011 \\ \mathrm{C} & 0.804696 & -0.727381 & 0.25806 \\ \mathrm{C} & 2.186446 & -0.999338 & -0.08384 \\ \mathrm{H} & 0.1626 & 2.52298 & -0.571078 \\ \mathrm{H} & 0.039039 & 0.238925 & -1.317791 \\ \mathrm{H} & 0.332019 & -1.455255 & 0.941169 \\ \mathrm{H} & 2.291251 & -2.068259 & -0.369237 \\ \mathrm{H} & 2.644472 & -0.33065 & -0.822921 \\ \mathrm{H} & 2.767068 & -0.976875 & 0.870243 \\ \mathrm{H} & 0.752185 & 2.028845 & 1.047575 \\ \mathrm{C} & -1.458719 & 0.387786 & 0.285351 \\ \mathrm{H} & -1.461807 & 0.451343 & 1.388458 \\ \mathrm{H} & -1.931426 & 1.316358 & -0.073698 \\ \mathrm{C} & -2.263025 & -0.825791 & -0.193749 \\ \mathrm{H} & -2.331691 & -0.859059 & -1.293537 \\ \mathrm{H} & -1.823484 & -1.782122 & 0.144341 \\ & -3.287874 & -0.790214 & 0.203356 \\ & 1.77039 & 1.762558 & -0.408568\end{array}$




$\begin{array}{llll}\text { 2_5CB_tt_CC } & & \\ \text { C } & 0.095206 & 1.536779 & 0.211129 \\ \mathrm{C} & 0.006739 & 0.023916 & -0.169309 \\ \mathrm{C} & 1.241925 & -0.67268 & 0.14738 \\ \mathrm{C} & 2.582654 & -0.245129 & -0.216656 \\ \mathrm{H} & -0.7835 & 2.033372 & -0.188945 \\ \mathrm{H} & 0.036516 & 0.008111 & -1.280415 \\ \mathrm{H} & 1.178056 & -1.603531 & 0.71424 \\ \mathrm{H} & 3.180845 & -1.1003 & -0.541905 \\ \mathrm{H} & 2.624848 & 0.584112 & -0.914618 \\ \mathrm{H} & 3.040175 & 0.054281 & 0.744029 \\ \mathrm{H} & 0.095162 & 1.639859 & 1.295188 \\ \mathrm{C} & -1.256296 & -0.685432 & 0.342014 \\ \mathrm{H} & -1.167331 & -1.754007 & 0.129178 \\ \mathrm{H} & -1.298762 & -0.578576 & 1.429247 \\ \mathrm{C} & -2.544966 & -0.15764 & -0.28877 \\ \mathrm{H} & -2.742863 & 0.876446 & -0.010725 \\ \mathrm{H} & -2.503511 & -0.22186 & -1.377284 \\ \mathrm{H} & -3.389798 & -0.755278 & 0.048202 \\ \mathrm{H} & 0.978588 & 2.018485 & -0.200913 \\ & & & \\ \mathrm{H} \text {-5CB_tt_PBE } & & \\ & & & \\ \mathrm{C} & 0.091269 & 1.574818 & 0.212326 \\ \mathrm{C} & 0.010962 & 0.029614 & -0.12087 \\ \mathrm{C} & 1.249802 & -0.651328 & 0.182712 \\ \mathrm{C} & 2.578476 & -0.273156 & -0.255199 \\ \mathrm{H} & -0.805869 & 2.045028 & -0.21071 \\ \mathrm{H} & 0.038114 & 0.039535 & -1.248322 \\ \mathrm{H} & 1.194057 & -1.565368 & 0.801234 \\ \mathrm{H} & 3.090006 & -1.161442 & -0.685622 \\ \mathrm{H} & 2.641568 & 0.596585 & -0.920606 \\ \mathrm{H} & 3.187868 & -0.104787 & 0.665419 \\ \mathrm{H} & 0.089974 & 1.701194 & 1.304533 \\ \mathrm{C} & -1.269659 & -0.662786 & 0.38361 \\ \mathrm{H} & -1.154854 & -1.754022 & 0.257565 \\ \mathrm{H} & -1.361382 & -0.477884 & 1.469005 \\ \mathrm{C} & -2.538678 & -0.200922 & -0.341969 \\ \mathrm{H} & -2.760791 & 0.862957 & -0.165414 \\ \mathrm{H} & -2.465229 & -0.362792 & -1.430641 \\ \mathrm{H} & -3.40472 & -0.776214 & 0.016995 \\ & 0.978223 & 2.059768 & -0.217103\end{array}$




\begin{tabular}{|c|c|c|c|}
\hline \multicolumn{4}{|c|}{ 2_5CB_tu_CC } \\
\hline C & -0.139834 & 1.612852 & -0.270561 \\
\hline C & -0.034293 & 0.275405 & 0.536565 \\
\hline C & -0.981135 & -0.706126 & 0.037507 \\
\hline C & -2.404365 & -0.4879 & -0.164821 \\
\hline $\mathrm{H}$ & 0.573393 & 2.305359 & 0.170783 \\
\hline $\mathrm{H}$ & -0.469515 & 0.519209 & 1.527207 \\
\hline $\mathrm{H}$ & -0.613186 & -1.701367 & -0.217448 \\
\hline $\mathrm{H}$ & -2.972792 & -1.350055 & 0.195667 \\
\hline $\mathrm{H}$ & -2.782258 & 0.451224 & 0.224736 \\
\hline $\mathrm{H}$ & -2.537054 & -0.519737 & -1.260926 \\
\hline $\mathrm{H}$ & 0.123193 & 1.45463 & -1.313835 \\
\hline C & 1.397968 & -0.253362 & 0.695846 \\
\hline $\mathrm{H}$ & 1.964423 & 0.491375 & 1.257001 \\
\hline $\mathrm{H}$ & 1.373675 & -1.151611 & 1.317779 \\
\hline C & 2.097637 & -0.549499 & -0.631852 \\
\hline $\mathrm{H}$ & 1.534751 & -1.263679 & -1.239457 \\
\hline $\mathrm{H}$ & 2.246524 & 0.35378 & -1.221841 \\
\hline $\mathrm{H}$ & 3.076459 & -0.987142 & -0.446054 \\
\hline $\mathrm{H}$ & -1.133481 & 2.049796 & -0.209714 \\
\hline \multicolumn{4}{|c|}{ 2_5CB_tu_PBE } \\
\hline C & -0.121422 & 1.643556 & -0.26527 \\
\hline C & -0.0385 & 0.265117 & 0.510516 \\
\hline C & -0.997292 & -0.693558 & 0.005966 \\
\hline C & -2.422866 & -0.487024 & -0.155528 \\
\hline $\mathrm{H}$ & 0.551538 & 2.338332 & 0.256992 \\
\hline $\mathrm{H}$ & -0.49524 & 0.539582 & 1.501394 \\
\hline $\mathrm{H}$ & -0.625458 & -1.695401 & -0.273826 \\
\hline $\mathrm{H}$ & -2.971162 & -1.328468 & 0.321777 \\
\hline $\mathrm{H}$ & -2.810699 & 0.486615 & 0.168323 \\
\hline $\mathrm{H}$ & -2.660645 & -0.663354 & -1.231875 \\
\hline $\mathrm{H}$ & 0.224594 & 1.507556 & -1.298839 \\
\hline $\mathrm{C}$ & 1.391647 & -0.271933 & 0.699473 \\
\hline $\mathrm{H}$ & 1.95337 & 0.469878 & 1.292443 \\
\hline $\mathrm{H}$ & 1.346034 & -1.184932 & 1.318507 \\
\hline $\mathrm{C}$ & 2.128076 & -0.557979 & -0.615246 \\
\hline $\mathrm{H}$ & 1.570718 & -1.269251 & -1.252583 \\
\hline $\mathrm{H}$ & 2.305674 & 0.355944 & -1.202399 \\
\hline $\mathrm{H}$ & 3.108397 & -1.012213 & -0.410565 \\
\hline $\mathrm{H}$ & -1.13498 & 2.066642 & -0.26881 \\
\hline
\end{tabular}




$\begin{array}{llll}\text { 3_6CA_gg_PBE } & & \\ \text { C } & 0.982529 & 0.03485 & -0.391364 \\ \text { C } & -0.122475 & -0.904002 & -0.37688 \\ \text { C } & -1.529135 & -0.790441 & -0.042276 \\ \text { H } & -2.073092 & -1.399124 & -0.805217 \\ \text { H } & 0.176483 & -1.931436 & -0.646536 \\ \text { C } & -2.24486 & 0.509958 & 0.303068 \\ \text { H } & -2.279924 & 1.191561 & -0.559268 \\ \text { H } & -3.282694 & 0.279182 & 0.583062 \\ \text { H } & -1.778897 & 1.03314 & 1.148896 \\ \text { H } & -1.591691 & -1.540229 & 0.798856 \\ \text { C } & 0.767104 & 1.52858 & -0.155651 \\ \text { H } & -0.011225 & 1.947866 & -0.807796 \\ \text { H } & 0.505459 & 1.738486 & 0.892412 \\ \text { H } & 1.705582 & 2.061194 & -0.370106 \\ \text { C } & 2.142235 & -0.572119 & 0.504305 \\ \text { H } & 3.036958 & 0.039785 & 0.321495 \\ \text { H } & 1.863188 & -0.497025 & 1.564923 \\ \text { H } & 2.372487 & -1.619034 & 0.257572 \\ \text { H } & 1.384972 & -0.145324 & -1.425505 \\ & & & \\ \text { 3_6CA_gg_CC } & & \\ \text { H } & & & \\ \text { C } & 0.987552 & 0.051714 & -0.410027 \\ \text { C } & -0.117232 & -0.897227 & -0.399796 \\ \text { C } & -1.501844 & -0.814783 & 0.025203 \\ \text { H } & -2.080274 & -1.554761 & -0.537275 \\ \text { H } & 0.181478 & -1.902577 & -0.699969 \\ \text { C } & -2.25041 & 0.498022 & 0.23173 \\ \text { H } & -2.351032 & 1.035955 & -0.708934 \\ \text { H } & -3.250108 & 0.265112 & 0.591855 \\ \text { H } & -1.768847 & 1.140828 & 0.961676 \\ \text { H } & -1.346105 & -1.349932 & 0.997923 \\ \text { C } & 0.738357 & 1.524435 & -0.101396 \\ \text { H } & -0.042931 & 1.950603 & -0.725871 \\ \text { H } & 0.480099 & 1.669781 & 0.94653 \\ \text { H } & 1.657637 & 2.074928 & -0.293733 \\ \text { C } & 2.131801 & -0.561932 & 0.468764 \\ \text { H } & 3.010036 & 0.065678 & 0.337376 \\ \text { H } & 1.845359 & -0.547663 & 1.519224 \\ & 2.381759 & -1.579458 & 0.172425 \\ & 1.353589 & -0.069875 & -1.448093\end{array}$




$\begin{array}{llll}\text { 3_6CA_gu_PBE } & & \\ \text { C } & -1.082827 & 0.016139 & -0.280984 \\ \text { C } & 0.307022 & 0.403263 & -0.371518 \\ \text { C } & 1.485427 & -0.429667 & -0.208876 \\ \text { H } & 1.717524 & -0.707633 & -1.27363 \\ \text { H } & 0.48904 & 1.448557 & -0.677596 \\ \text { C } & -1.427238 & -1.393127 & 0.197322 \\ \text { H } & -0.856714 & -2.172626 & -0.329154 \\ \text { H } & -1.237446 & -1.487178 & 1.279122 \\ \text { H } & -2.496046 & -1.590729 & 0.032146 \\ \text { C } & -2.02611 & 1.139235 & 0.2451 \\ \text { H } & -3.060256 & 0.900525 & -0.037091 \\ \text { H } & -1.958643 & 1.171348 & 1.343129 \\ \text { H } & -1.768892 & 2.130748 & -0.157005 \\ \text { H } & -1.232931 & 0.081314 & -1.411521 \\ \text { C } & 2.73995 & 0.29863 & 0.336757 \\ \text { H } & 3.602091 & -0.378613 & 0.266671 \\ \text { H } & 2.967497 & 1.210127 & -0.236531 \\ \text { H } & 2.594019 & 0.567501 & 1.392623 \\ \text { H } & 1.263416 & -1.380184 & 0.302029 \\ & & & \\ \text { 3_6CA_gu_CC } & & \\ \text { H } & & & \\ \text { C } & 1.08634 & -0.016007 & -0.296669 \\ \text { C } & -0.303211 & -0.411463 & -0.358792 \\ \text { C } & -1.486695 & 0.429226 & -0.233397 \\ \text { H } & -1.700374 & 0.673364 & -1.293777 \\ \text { H } & -0.483559 & -1.452625 & -0.632757 \\ \text { C } & 1.405055 & 1.387778 & 0.210127 \\ \text { H } & 0.837931 & 2.155453 & -0.313251 \\ \text { H } & 1.19834 & 1.45952 & 1.27829 \\ \text { H } & 2.462989 & 1.591437 & 0.059338 \\ \text { C } & 2.020799 & -1.123378 & 0.242041 \\ \text { H } & 3.048029 & -0.869325 & -0.006784 \\ \text { H } & 1.931106 & -1.179423 & 1.325914 \\ \text { H } & 1.788757 & -2.097414 & -0.186943 \\ \text { H } & 1.209336 & -0.050644 & -1.415393 \\ \text { C } & -2.715529 & -0.299714 & 0.346155 \\ \text { H } & -3.574181 & 0.364818 & 0.292423 \\ \text { H } & -2.941626 & -1.203411 & -0.218091 \\ \text { H } & -2.550143 & -0.561531 & 1.389287 \\ & -1.267154 & 1.371136 & 0.264952\end{array}$


3_6CA_tg_PBE

$\begin{array}{llll}\mathrm{C} & -1.03117 & -0.070597 & -0.311386 \\ \mathrm{H} & -0.851648 & -0.033598 & -1.402948 \\ \mathrm{C} & 0.29859 & -0.375881 & 0.204561 \\ \mathrm{H} & 0.428052 & -1.28759 & 0.792955 \\ \mathrm{C} & 1.482979 & 0.445167 & 0.04924 \\ \mathrm{H} & 1.351965 & 1.23759 & -0.6859 \\ \mathrm{H} & 1.471618 & 0.937103 & 1.04978 \\ \mathrm{C} & 2.810616 & -0.321658 & -0.089046 \\ \mathrm{H} & 2.858996 & -0.821852 & -1.05424 \\ \mathrm{H} & 3.635185 & 0.383623 & -0.025479 \\ \mathrm{H} & 2.924723 & -1.060941 & 0.702525 \\ \mathrm{C} & -2.090777 & -1.118496 & 0.029645 \\ \mathrm{H} & -3.016309 & -0.888725 & -0.494407 \\ \mathrm{H} & -1.775336 & -2.118251 & -0.267514 \\ \mathrm{H} & -2.300548 & -1.118201 & 1.099578 \\ \mathrm{C} & -1.463747 & 1.378517 & 0.079919 \\ \mathrm{H} & -0.749157 & 2.129465 & -0.248326 \\ \mathrm{H} & -2.418844 & 1.572217 & -0.402184 \\ \mathrm{H} & -1.597641 & 1.446848 & 1.158568\end{array}$

3_6CA_tg_CC

$\begin{array}{llll}\mathrm{C} & -0.9017 & 0.029287 & -0.256892 \\ \mathrm{H} & -0.618829 & 0.076756 & -1.337042 \\ \mathrm{C} & 0.12756 & -0.912334 & 0.160387 \\ \mathrm{H} & -0.217596 & -1.915068 & 0.42094 \\ \mathrm{C} & 1.575279 & -0.77195 & 0.215197 \\ \mathrm{H} & 1.78476 & -0.998662 & 1.280994 \\ \mathrm{H} & 1.961485 & -1.687804 & -0.260053 \\ \mathrm{C} & 2.294638 & 0.481688 & -0.266857 \\ \mathrm{H} & 2.158611 & 1.316639 & 0.414989 \\ \mathrm{H} & 3.359912 & 0.268796 & -0.322487 \\ \mathrm{H} & 1.96054 & 0.774169 & -1.261945 \\ \mathrm{C} & -2.323064 & -0.526579 & -0.125011 \\ \mathrm{H} & -3.017697 & 0.109961 & -0.668934 \\ \mathrm{H} & -2.402384 & -1.535325 & -0.529445 \\ \mathrm{H} & -2.627882 & -0.540341 & 0.921531 \\ \mathrm{C} & -0.775907 & 1.475246 & 0.287635 \\ \mathrm{H} & 0.15194 & 1.95979 & 0.006955 \\ \mathrm{H} & -1.602323 & 2.049249 & -0.124812 \\ \mathrm{H} & -0.871369 & 1.469695 & 1.372563\end{array}$


3_6CA_tt_PBE

$\begin{array}{llll}\mathrm{C} & -1.020989 & -0.083177 & -0.293605 \\ \mathrm{H} & -0.82603 & -0.00894 & -1.395061 \\ \mathrm{C} & 0.299951 & -0.341927 & 0.252384 \\ \mathrm{H} & 0.424311 & -1.226995 & 0.903487 \\ \mathrm{C} & 1.491688 & 0.457389 & 0.060476 \\ \mathrm{H} & 1.362075 & 1.273628 & -0.667732 \\ \mathrm{H} & 1.560807 & 0.943268 & 1.075727 \\ \mathrm{C} & 2.81512 & -0.333856 & -0.116626 \\ \mathrm{H} & 2.833912 & -0.808348 & -1.108421 \\ \mathrm{H} & 3.659326 & 0.365052 & -0.045076 \\ \mathrm{H} & 2.936953 & -1.110081 & 0.653899 \\ \mathrm{C} & -2.082911 & -1.143156 & 0.011651 \\ \mathrm{H} & -2.999556 & -0.93245 & -0.558267 \\ \mathrm{H} & -1.741366 & -2.152802 & -0.26274 \\ \mathrm{H} & -2.347363 & -1.139973 & 1.081031 \\ \mathrm{C} & -1.497057 & 1.384741 & 0.066483 \\ \mathrm{H} & -0.773508 & 2.154474 & -0.234645 \\ \mathrm{H} & -2.439011 & 1.551935 & -0.474523 \\ \mathrm{H} & -1.685363 & 1.451145 & 1.147738\end{array}$

3_6CA_tt_CC

$\begin{array}{llll}\mathrm{C} & -1.03117 & -0.070597 & -0.311386 \\ \mathrm{H} & -0.851648 & -0.033598 & -1.402948 \\ \mathrm{C} & 0.29859 & -0.375881 & 0.204561 \\ \mathrm{H} & 0.428052 & -1.28759 & 0.792955 \\ \mathrm{C} & 1.482979 & 0.445167 & 0.04924 \\ \mathrm{H} & 1.351965 & 1.23759 & -0.6859 \\ \mathrm{H} & 1.471618 & 0.937103 & 1.04978 \\ \mathrm{C} & 2.810616 & -0.321658 & -0.089046 \\ \mathrm{H} & 2.858996 & -0.821852 & -1.05424 \\ \mathrm{H} & 3.635185 & 0.383623 & -0.025479 \\ \mathrm{H} & 2.924723 & -1.060941 & 0.702525 \\ \mathrm{C} & -2.090777 & -1.118496 & 0.029645 \\ \mathrm{H} & -3.016309 & -0.888725 & -0.494407 \\ \mathrm{H} & -1.775336 & -2.118251 & -0.267514 \\ \mathrm{H} & -2.300548 & -1.118201 & 1.099578 \\ \mathrm{C} & -1.463747 & 1.378517 & 0.079919 \\ \mathrm{H} & -0.749157 & 2.129465 & -0.248326 \\ \mathrm{H} & -2.418844 & 1.572217 & -0.402184 \\ \mathrm{H} & -1.597641 & 1.446848 & 1.158568\end{array}$


3_6CA_tu_PBE

$\begin{array}{llll}\mathrm{C} & -1.029662 & -0.053119 & -0.316311 \\ \mathrm{H} & -1.109417 & -0.060411 & -1.436575 \\ \mathrm{C} & 0.245496 & -0.714721 & -0.103434 \\ \mathrm{H} & 0.262505 & -1.59731 & 0.561282 \\ \mathrm{C} & 1.528309 & -0.321412 & -0.646894 \\ \mathrm{H} & 2.029674 & -1.218588 & -1.063646 \\ \mathrm{H} & 1.46971 & 0.471663 & -1.40616 \\ \mathrm{C} & 2.425579 & 0.125011 & 0.581669 \\ \mathrm{H} & 2.545773 & -0.682819 & 1.317525 \\ \mathrm{H} & 3.412022 & 0.372088 & 0.161577 \\ \mathrm{H} & 2.009359 & 1.015214 & 1.071719 \\ \mathrm{C} & -2.244001 & -0.727526 & 0.32722 \\ \mathrm{H} & -3.170801 & -0.253208 & -0.027012 \\ \mathrm{H} & -2.297327 & -1.797832 & 0.076355 \\ \mathrm{H} & -2.215677 & -0.624365 & 1.423707 \\ \mathrm{C} & -0.941495 & 1.493153 & 0.003872 \\ \mathrm{H} & -0.112417 & 1.99191 & -0.516777 \\ \mathrm{H} & -1.888086 & 1.941648 & -0.32846 \\ \mathrm{H} & -0.840678 & 1.633697 & 1.08974\end{array}$

3_6CA_tu_CC

$\begin{array}{llll}\mathrm{C} & -1.022191 & -0.049105 & -0.335868 \\ \mathrm{H} & -1.11437 & -0.114734 & -1.437098 \\ \mathrm{C} & 0.237583 & -0.744749 & -0.100138 \\ \mathrm{H} & 0.232421 & -1.613527 & 0.561558 \\ \mathrm{C} & 1.531089 & -0.372227 & -0.636979 \\ \mathrm{H} & 2.045224 & -1.269226 & -0.993207 \\ \mathrm{H} & 1.471181 & 0.377395 & -1.421648 \\ \mathrm{C} & 2.358201 & 0.162499 & 0.593004 \\ \mathrm{H} & 2.466069 & -0.600282 & 1.361369 \\ \mathrm{H} & 3.343293 & 0.416907 & 0.208662 \\ \mathrm{H} & 1.90504 & 1.05399 & 1.019067 \\ \mathrm{C} & -2.230042 & -0.685296 & 0.35158 \\ \mathrm{H} & -3.142327 & -0.19145 & 0.022952 \\ \mathrm{H} & -2.315702 & -1.74449 & 0.110679 \\ \mathrm{H} & -2.158063 & -0.574578 & 1.433893 \\ \mathrm{C} & -0.88915 & 1.478017 & -0.034764 \\ \mathrm{H} & -0.072631 & 1.941348 & -0.583816 \\ \mathrm{H} & -1.822031 & 1.947677 & -0.337029 \\ \mathrm{H} & -0.751048 & 1.636137 & 1.03361\end{array}$


3_6CB_55_PBE

$\begin{array}{llll}\mathrm{C} & -1.633658 & 0.417312 & -0.549544 \\ \mathrm{H} & -1.510515 & 1.480942 & -0.318582 \\ \mathrm{C} & -0.42401 & -0.340591 & -0.707573 \\ \mathrm{H} & -0.530962 & -1.352042 & -1.132684 \\ \mathrm{C} & 0.940757 & 0.065551 & -0.365799 \\ \mathrm{H} & 1.252086 & 0.194777 & -1.449117 \\ \mathrm{C} & 1.821975 & -1.079681 & 0.180948 \\ \mathrm{H} & 1.68433 & -2.019193 & -0.37642 \\ \mathrm{H} & 2.881833 & -0.795857 & 0.121686 \\ \mathrm{H} & 1.578696 & -1.260229 & 1.240929 \\ \mathrm{C} & -1.92158 & -0.439526 & 0.788101 \\ \mathrm{H} & -1.050361 & -0.968782 & 1.228785 \\ \mathrm{H} & -2.226791 & 0.293927 & 1.548739 \\ \mathrm{H} & -2.69159 & -1.190951 & 0.579226 \\ \mathrm{H} & -2.429826 & 0.225689 & -1.278682 \\ \mathrm{C} & 1.127456 & 1.404142 & 0.353487 \\ \mathrm{H} & 0.768863 & 1.337892 & 1.394039 \\ \mathrm{H} & 2.197423 & 1.653767 & 0.391776 \\ \mathrm{H} & 0.611176 & 2.23681 & -0.147412\end{array}$

3_6CB_55_CC_mod

$\begin{array}{llll}\mathrm{C} & -1.610569 & 0.386479 & -0.56205 \\ \mathrm{H} & -1.492982 & 1.444508 & -0.363242 \\ \mathrm{C} & -0.3974 & -0.350538 & -0.732045 \\ \mathrm{H} & -0.497012 & -1.357933 & -1.135977 \\ \mathrm{C} & 0.96194 & 0.069342 & -0.384732 \\ \mathrm{H} & 1.306978 & 0.200185 & -1.438101 \\ \mathrm{C} & 1.808778 & -1.068573 & 0.214742 \\ \mathrm{H} & 1.709876 & -1.992673 & -0.354298 \\ \mathrm{H} & 2.856195 & -0.77611 & 0.214948 \\ \mathrm{H} & 1.507839 & -1.2542 & 1.246262 \\ \mathrm{C} & -1.931312 & -0.418082 & 0.795951 \\ \mathrm{H} & -1.053644 & -0.774625 & 1.342483 \\ \mathrm{H} & -2.427249 & 0.306338 & 1.437798 \\ \mathrm{H} & -2.575484 & -1.26228 & 0.574805 \\ \mathrm{H} & -2.394531 & 0.171808 & -1.280748 \\ \mathrm{C} & 1.101771 & 1.399055 & 0.354267 \\ \mathrm{H} & 0.682926 & 1.326161 & 1.35913 \\ \mathrm{H} & 2.157711 & 1.641616 & 0.452727 \\ \mathrm{H} & 0.62013 & 2.221101 & -0.172578\end{array}$




$\begin{array}{llll}\text { 7_6CA_gg_PBE } & & \\ \text { C } & -0.82958 & 0.043854 & -0.290692 \\ \text { C } & -1.307867 & 1.426104 & 0.211712 \\ \text { C } & 0.242097 & -0.442984 & 0.672344 \\ \text { C } & 1.647174 & -0.2722 & 0.539944 \\ \text { H } & -0.491321 & 2.164892 & 0.238036 \\ \text { H } & -1.749671 & 1.356973 & 1.219286 \\ \text { H } & -2.087585 & 1.801718 & -0.46856 \\ \text { H } & -0.099306 & -0.646418 & 1.701147 \\ \text { H } & 0.668075 & -1.51216 & 0.344089 \\ \text { C } & -1.986696 & -0.967932 & -0.368674 \\ \text { H } & -2.770118 & -0.583283 & -1.038377 \\ \text { H } & -1.659305 & -1.943343 & -0.763658 \\ \text { H } & -2.444434 & -1.130149 & 0.621379 \\ \text { H } & -0.39132 & 0.164456 & -1.297244 \\ \text { C } & 2.385081 & 0.187946 & -0.639745 \\ \text { H } & 1.772324 & 0.382839 & -1.528369 \\ \text { H } & 2.854669 & 1.145667 & -0.317008 \\ \text { H } & 3.244975 & -0.466069 & -0.867158 \\ \text { H } & 2.251767 & -0.583847 & 1.407092 \\ & & & \\ \text { 7_6CA_gg_CC } & & \\ \text { H } & & & \\ \text { C } & -0.827495 & 0.066144 & -0.290489 \\ \text { C } & -1.097013 & 1.434144 & 0.351306 \\ \text { C } & 0.217946 & -0.667577 & 0.544017 \\ \text { C } & 1.615167 & -0.427676 & 0.473627 \\ \text { H } & -0.196603 & 2.049897 & 0.38877 \\ \text { H } & -1.478474 & 1.317634 & 1.366932 \\ \text { H } & -1.846266 & 1.969465 & -0.22963 \\ \text { H } & -0.12118 & -1.016051 & 1.519973 \\ \text { H } & 0.562243 & -1.646805 & 0.024152 \\ \text { C } & -2.100179 & -0.779716 & -0.389567 \\ \text { H } & -2.847185 & -0.252926 & -0.980906 \\ \text { H } & -1.908971 & -1.740994 & -0.868257 \\ \text { H } & -2.522433 & -0.96306 & 0.599896 \\ \text { H } & -0.433492 & 0.217819 & -1.298113 \\ \text { C } & 2.340639 & 0.284878 & -0.583784 \\ \text { H } & 1.722145 & 0.601448 & -1.416092 \\ \text { H } & 2.739223 & 1.1757 & -0.0736 \\ & 3.213942 & -0.279681 & -0.91235 \\ & 2.222655 & -0.893632 & 1.248567\end{array}$




\begin{tabular}{|c|c|c|c|}
\hline \multicolumn{4}{|c|}{ 7_6CA_tt_PBE } \\
\hline C & 1.167261 & -0.038816 & 0.428639 \\
\hline C & 1.754633 & -1.190686 & -0.422457 \\
\hline C & 1.480145 & 1.341881 & -0.156717 \\
\hline $\mathrm{C}$ & -0.32513 & -0.323875 & 0.596152 \\
\hline $\mathrm{C}$ & -1.362221 & 0.30713 & -0.137149 \\
\hline $\mathrm{C}$ & -2.757253 & -0.141753 & -0.212476 \\
\hline $\mathrm{H}$ & 1.344338 & -1.176506 & -1.445582 \\
\hline $\mathrm{H}$ & 1.560761 & -2.180097 & 0.020786 \\
\hline $\mathrm{H}$ & 2.845819 & -1.062294 & -0.487891 \\
\hline $\mathrm{H}$ & 1.610976 & -0.111982 & 1.438389 \\
\hline $\mathrm{H}$ & 1.143565 & 1.434291 & -1.203738 \\
\hline $\mathrm{H}$ & 1.037686 & 2.163316 & 0.432777 \\
\hline $\mathrm{H}$ & 2.568617 & 1.500133 & -0.158071 \\
\hline $\mathrm{H}$ & -0.591836 & -1.287777 & 1.058107 \\
\hline $\mathrm{H}$ & -0.794167 & 0.499829 & 1.324225 \\
\hline $\mathrm{H}$ & -1.104215 & 1.252649 & -0.640653 \\
\hline $\mathrm{H}$ & -2.909052 & -0.415358 & -1.281496 \\
\hline $\mathrm{H}$ & -2.98559 & -1.020745 & 0.405649 \\
\hline $\mathrm{H}$ & -3.471505 & 0.681253 & -0.038452 \\
\hline \multicolumn{4}{|c|}{ 7_6CA_tt_CC } \\
\hline $\mathrm{C}$ & 1.16848 & -0.053242 & 0.434381 \\
\hline $\mathrm{C}$ & 1.601352 & -1.258439 & -0.411097 \\
\hline $\mathrm{C}$ & 1.562285 & 1.275808 & -0.211846 \\
\hline $\mathrm{C}$ & -0.336347 & -0.169277 & 0.71644 \\
\hline $\mathrm{C}$ & -1.320436 & 0.300295 & -0.194183 \\
\hline $\mathrm{C}$ & -2.72439 & -0.116939 & -0.214277 \\
\hline $\mathrm{H}$ & 1.133456 & -1.227081 & -1.397925 \\
\hline $\mathrm{H}$ & 1.339666 & -2.202598 & 0.067258 \\
\hline $\mathrm{H}$ & 2.68108 & -1.240145 & -0.550186 \\
\hline $\mathrm{H}$ & 1.654439 & -0.122231 & 1.409997 \\
\hline $\mathrm{H}$ & 1.187767 & 1.359627 & -1.234316 \\
\hline $\mathrm{H}$ & 1.210694 & 2.133816 & 0.364674 \\
\hline $\mathrm{H}$ & 2.647428 & 1.341818 & -0.266591 \\
\hline $\mathrm{H}$ & -0.643384 & -1.039071 & 1.296275 \\
\hline $\mathrm{H}$ & -0.674812 & 0.740071 & 1.334636 \\
\hline $\mathrm{H}$ & -1.027173 & 1.092591 & -0.88089 \\
\hline $\mathrm{H}$ & -2.823202 & -0.65958 & -1.168352 \\
\hline $\mathrm{H}$ & -2.99323 & -0.783232 & 0.600271 \\
\hline $\mathrm{H}$ & -3.398393 & 0.736783 & -0.291356 \\
\hline
\end{tabular}




$\begin{array}{llll}\text { 7_6CA_gt_PBE } & & \\ \text { C } & -1.062576 & -0.000986 & -0.339006 \\ \text { C } & -1.495049 & 1.411261 & 0.115215 \\ \text { C } & 0.315237 & -0.26555 & 0.260681 \\ \text { C } & 1.54081 & -0.044697 & -0.41661 \\ \text { C } & 2.872854 & 0.000193 & 0.195558 \\ \text { H } & -0.796504 & 2.190029 & -0.22945 \\ \text { H } & -1.57934 & 1.474886 & 1.212632 \\ \text { H } & -2.488123 & 1.629543 & -0.307133 \\ \text { H } & 0.375256 & -0.309022 & 1.36095 \\ \text { H } & 0.691091 & -1.343193 & -0.098022 \\ \text { H } & 1.483069 & 0.041977 & -1.514829 \\ \text { H } & 3.21919 & 1.047429 & 0.038028 \\ \text { H } & 2.883899 & -0.2098 & 1.273741 \\ \text { H } & 3.607423 & -0.613914 & -0.353222 \\ \text { C } & -2.072001 & -1.077099 & 0.093275 \\ \text { H } & -3.063982 & -0.84174 & -0.319981 \\ \text { H } & -1.789558 & -2.079541 & -0.267205 \\ \text { H } & -2.169913 & -1.117296 & 1.190854 \\ \text { H } & -0.968153 & -0.008091 & -1.441042 \\ & & & \\ \text { 7_6CA_gt_CC } & & \\ \text { H } & & & \\ \text { C } & -1.057405 & 0.00733 & -0.337041 \\ \text { C } & -1.378728 & 1.427935 & 0.143844 \\ \text { C } & 0.30755 & -0.401291 & 0.222927 \\ \text { C } & 1.517058 & -0.026804 & -0.416647 \\ \text { C } & 2.845267 & -0.014958 & 0.202399 \\ \text { H } & -0.630001 & 2.147556 & -0.192808 \\ \text { H } & -1.431829 & 1.467372 & 1.233134 \\ \text { H } & -2.34498 & 1.738341 & -0.250538 \\ \text { H } & 0.372684 & -0.503953 & 1.306354 \\ \text { H } & 0.594906 & -1.438472 & -0.193783 \\ \text { H } & 1.458681 & 0.197696 & -1.481956 \\ \text { H } & 3.112594 & 1.053505 & 0.230939 \\ \text { H } & 2.856023 & -0.408353 & 1.214776 \\ \text { H } & 3.592489 & -0.48943 & -0.434206 \\ \text { C } & -2.12967 & -0.998274 & 0.087295 \\ \text { H } & -3.097604 & -0.693843 & -0.307257 \\ \text { H } & -1.913733 & -1.999871 & -0.286795 \\ \text { H } & -2.209645 & -1.044064 & 1.174719 \\ & -0.984021 & 0.009883 & -1.42924\end{array}$




$\begin{array}{llll}\text { 9_8_gd_PBE } & & \\ \text { C } & 0.502385 & -0.361982 & 0.612861 \\ \text { C } & 1.514769 & -0.06733 & -0.528456 \\ \text { C } & -0.740271 & -0.992854 & 0.037653 \\ \text { C } & -1.953399 & -0.331538 & -0.289802 \\ \text { H } & 0.27371 & 0.563025 & 1.166688 \\ \text { H } & 1.719565 & -0.997995 & -1.086775 \\ \text { H } & 1.055536 & 0.637818 & -1.244375 \\ \text { H } & -0.613601 & -1.979763 & -0.434511 \\ \text { H } & -1.576379 & -1.223965 & 0.887024 \\ \text { H } & 0.959953 & -1.067625 & 1.326827 \\ \text { C } & 2.821328 & 0.509065 & 0.02583 \\ \text { H } & 3.519827 & 0.719236 & -0.798462 \\ \text { H } & 2.650053 & 1.451692 & 0.570253 \\ \text { H } & 3.315117 & -0.198187 & 0.711213 \\ \text { C } & -2.342635 & 1.044036 & 0.054994 \\ \text { H } & -1.616286 & 1.590119 & 0.669548 \\ \text { H } & -2.44188 & 1.566665 & -0.92193 \\ \text { H } & -3.354877 & 1.084647 & 0.492498 \\ \text { H } & -2.703804 & -0.942047 & -0.816471\end{array}$

\section{9_8_gd_CC}

$\begin{array}{llll}\mathrm{C} & 0.502244 & -0.561251 & 0.575626 \\ \mathrm{C} & 1.394041 & 0.047738 & -0.518251 \\ \mathrm{C} & -0.799043 & -1.073291 & -0.01777 \\ \mathrm{C} & -1.908863 & -0.245214 & -0.323344 \\ \mathrm{H} & 0.310041 & 0.170434 & 1.361766 \\ \mathrm{H} & 1.550866 & -0.691267 & -1.308255 \\ \mathrm{H} & 0.874108 & 0.894593 & -0.977443 \\ \mathrm{H} & -0.724915 & -1.962009 & -0.642836 \\ \mathrm{H} & -1.556206 & -1.394167 & 0.806944 \\ \mathrm{H} & 1.010526 & -1.404009 & 1.045432 \\ \mathrm{C} & 2.740318 & 0.504818 & 0.040044 \\ \mathrm{H} & 3.352683 & 0.936685 & -0.749789 \\ \mathrm{H} & 2.607774 & 1.259632 & 0.816054 \\ \mathrm{H} & 3.287648 & -0.334146 & 0.470386 \\ \mathrm{C} & -2.14906 & 1.123122 & 0.152344 \\ \mathrm{H} & -1.399146 & 1.493056 & 0.842727 \\ \mathrm{H} & -2.125366 & 1.732199 & -0.763828 \\ \mathrm{H} & -3.158767 & 1.229267 & 0.55067 \\ \mathrm{H} & -2.707066 & -0.7058 & -0.903726\end{array}$




$\begin{array}{llll}\text { 9_8_gg_PBE } & & \\ \text { C } & 0.603205 & 0.897368 & 0.198651 \\ \text { C } & 1.235496 & -0.468659 & -0.113854 \\ \text { C } & -0.873938 & 1.14341 & -0.054989 \\ \text { C } & -1.981778 & 0.249644 & -0.010225 \\ \text { H } & 1.142628 & 1.686927 & -0.354728 \\ \text { H } & 0.867576 & -1.232508 & 0.593173 \\ \text { H } & 0.946042 & -0.795673 & -1.130218 \\ \text { H } & -1.177464 & 2.197863 & 0.049685 \\ \text { H } & -1.167989 & 0.93001 & -1.196276 \\ \text { H } & 0.754493 & 1.154285 & 1.266672 \\ \text { C } & 2.76525 & -0.401188 & -0.015778 \\ \text { H } & 3.210712 & -1.384475 & -0.226565 \\ \text { H } & 3.18163 & 0.318467 & -0.739113 \\ \text { H } & 3.089324 & -0.096739 & 0.992496 \\ \text { C } & -2.018084 & -1.212724 & 0.066104 \\ \text { H } & -1.047123 & -1.715736 & 0.020331 \\ \text { H } & -2.473066 & -1.413613 & 1.064818 \\ \text { H } & -2.74539 & -1.638991 & -0.646367 \\ \text { H } & -2.962286 & 0.743085 & -0.113362 \\ & & & \\ \text { 9_8_gg_CC } & & & \\ \text { H } & 0.487974 & 0.618639 & 0.525509 \\ \text { C } & 1.395226 & -0.05068 & -0.51752 \\ \text { C } & -0.852083 & 1.092797 & -0.037735 \\ \text { C } & -2.000851 & 0.262485 & -0.102314 \\ \text { C } & 0.991777 & 1.493809 & 0.935838 \\ \text { H } & 0.884669 & -0.914675 & -0.95507 \\ \text { H } & 1.573676 & 0.653667 & -1.335013 \\ \text { H } & -1.083641 & 2.154471 & 0.039249 \\ \text { H } & -2.614963 & -1.678763 & -0.696452 \\ \text { H } & -0.903339 & 0.921767 & -1.184268 \\ \text { H } & 0.307847 & -0.057348 & 1.364612 \\ \text { C } & 2.727561 & -0.491337 & 0.084617 \\ \text { H } & 3.356318 & -0.952849 & -0.674821 \\ \text { H } & 3.267715 & 0.361352 & 0.496714 \\ \text { H } & 2.575974 & -1.216965 & 0.884736 \\ \text { C } & -2.043283 & -1.189803 & 0.093327 \\ \text { H } & -1.070695 & -1.647205 & 0.235949 \\ & -2.640639 & -1.314415 & 1.010306 \\ \text { H } & 0.744546 & -0.397082\end{array}$


9_8_tt_PBE

$\begin{array}{llll}\text { C } & 0.660753 & -0.397096 & 0.248149 \\ \text { C } & 1.824723 & 0.509237 & -0.191324 \\ \text { C } & -0.696844 & 0.282334 & 0.17126 \\ \text { C } & -1.901783 & -0.386021 & -0.172413 \\ \text { C } & -3.258094 & 0.12828 & 0.01654 \\ \text { H } & 0.792166 & -0.684973 & 1.309344 \\ \text { H } & 1.685568 & 0.791726 & -1.251709 \\ \text { H } & 1.795416 & 1.449082 & 0.389487 \\ \text { H } & -0.817769 & 1.222102 & 0.734872 \\ \text { H } & -0.882969 & 0.608594 & -0.95661 \\ \text { H } & -1.793779 & -1.356286 & -0.686361 \\ \text { H } & -3.308148 & 1.148835 & 0.419762 \\ \text { H } & -3.71454 & -0.566242 & 0.760774 \\ \text { H } & 0.651423 & -1.336125 & -0.333747 \\ \text { H } & -3.884957 & 0.00153 & -0.883329 \\ \text { C } & 3.182454 & -0.175335 & -0.008324 \\ \text { H } & 3.997064 & 0.489162 & -0.331861 \\ \text { H } & 3.362486 & -0.434308 & 1.047945 \\ \text { H } & 3.250787 & -1.101489 & -0.601899\end{array}$

\section{9_8_tt_CC}

$\begin{array}{llll}\text { C } & 0.649374 & -0.398792 & 0.192092 \\ \text { C } & 1.827848 & 0.513633 & -0.159547 \\ \text { C } & -0.692779 & 0.320003 & 0.074536 \\ \text { C } & -1.898174 & -0.389578 & -0.174152 \\ \text { C } & -3.250882 & 0.11372 & 0.070037 \\ \text { H } & 0.739382 & -0.747534 & 1.223847 \\ \text { H } & 1.727955 & 0.84598 & -1.197105 \\ \text { H } & 1.787662 & 1.410321 & 0.464699 \\ \text { H } & -0.811442 & 1.225252 & 0.669384 \\ \text { H } & -0.832989 & 0.667018 & -1.014421 \\ \text { H } & -1.80558 & -1.362497 & -0.657114 \\ \text { H } & -3.278489 & 1.150573 & 0.392392 \\ \text { H } & -3.622894 & -0.52508 & 0.887729 \\ \text { H } & 0.649787 & -1.284127 & -0.449228 \\ \text { H } & -3.912473 & -0.077126 & -0.77541 \\ \text { C } & 3.168994 & -0.191804 & 0.032535 \\ \text { H } & 3.990572 & 0.473383 & -0.22737 \\ \text { H } & 3.30214 & -0.501788 & 1.069623 \\ \text { H } & 3.240081 & -1.077467 & -0.600027\end{array}$


8_8_dgd_PBE

$\begin{array}{llll}\text { C } & 1.621649 & -0.177523 & 0.573571 \\ \text { C } & 0.707203 & -0.911765 & -0.351919 \\ \text { C } & -0.707203 & -0.911767 & -0.351919 \\ \text { H } & 1.180447 & -1.449697 & -1.188044 \\ \text { H } & -0.000005 & -1.868737 & 0.229206 \\ \text { H } & 1.088134 & 0.225731 & 1.447212 \\ \text { H } & -1.180446 & -1.449696 & -1.188046 \\ \text { C } & -1.62165 & -0.177523 & 0.57357 \\ \text { H } & -1.088136 & 0.22573 & 1.447212 \\ \text { C } & -2.29623 & 0.966113 & -0.231283 \\ \text { H } & -2.864912 & 0.581973 & -1.092023 \\ \text { H } & -3.000981 & 1.486522 & 0.433627 \\ \text { H } & -1.557605 & 1.697319 & -0.592472 \\ \text { H } & -2.402525 & -0.868424 & 0.934885 \\ \text { C } & 2.29623 & 0.966112 & -0.231283 \\ \text { H } & 3.000981 & 1.486521 & 0.433627 \\ \text { H } & 2.864914 & 0.581971 & -1.092022 \\ \text { H } & 1.557607 & 1.697318 & -0.592473 \\ \text { H } & 2.402524 & -0.868424 & 0.934886\end{array}$

8_8_dgu_PBE

$\begin{array}{llll}\text { C } & -1.45574 & -0.533612 & -0.263993 \\ \mathrm{C} & -2.899605 & -0.263109 & 0.192112 \\ \mathrm{C} & -0.61629 & 0.710506 & -0.347885 \\ \mathrm{C} & 0.733949 & 0.869025 & 0.047794 \\ \mathrm{H} & -1.463027 & -0.930529 & -1.299269 \\ \mathrm{H} & -2.933035 & 0.092292 & 1.234212 \\ \mathrm{H} & -3.394196 & 0.485533 & -0.446692 \\ \mathrm{H} & -3.485617 & -1.191338 & 0.135847 \\ \mathrm{H} & -1.045185 & 1.552279 & -0.914054 \\ \mathrm{H} & -0.429498 & 1.234228 & 0.779644 \\ \mathrm{H} & -0.974109 & -1.303284 & 0.359556 \\ \mathrm{H} & 1.178332 & 1.853492 & -0.168522 \\ \mathrm{C} & 1.620056 & -0.131656 & 0.687792 \\ \mathrm{H} & 1.074333 & -1.024833 & 1.024877 \\ \mathrm{C} & 2.691449 & -0.519243 & -0.379067 \\ \mathrm{H} & 3.277179 & 0.351895 & -0.709262 \\ \mathrm{H} & 3.382232 & -1.235927 & 0.089088 \\ \mathrm{H} & 2.234766 & -0.998975 & -1.256883 \\ \mathrm{H} & 2.134912 & 0.323705 & 1.550936\end{array}$




$\begin{array}{llll}\text { 8_8_dtg_PBE } & & \\ \text { C } & 1.90138 & -0.346699 & -0.481692 \\ \text { C } & 0.460673 & -0.022903 & -0.407683 \\ \text { C } & -0.551029 & -0.860126 & 0.119216 \\ \text { C } & -1.970402 & -0.44044 & 0.429458 \\ \text { H } & 0.136163 & 0.953289 & -0.801559 \\ \text { H } & -0.410436 & -0.986102 & -1.096682 \\ \text { H } & -0.21149 & -1.824855 & 0.527227 \\ \text { H } & -2.009953 & -0.401539 & 1.53663 \\ \text { H } & 2.099334 & -1.398565 & -0.222505 \\ \text { H } & -2.64156 & -1.273 & 0.155608 \\ \text { C } & -2.443858 & 0.880744 & -0.176955 \\ \text { H } & -1.858019 & 1.74168 & 0.184013 \\ \text { H } & -2.409864 & 0.867148 & -1.279735 \\ \text { H } & -3.490187 & 1.060675 & 0.108366 \\ \text { C } & 2.615057 & 0.607081 & 0.529384 \\ \text { H } & 3.6935 & 0.396779 & 0.473429 \\ \text { H } & 2.460865 & 1.666654 & 0.275861 \\ \text { H } & 2.27908 & 0.429 & 1.561184 \\ \text { H } & 2.291641 & -0.1371 & -1.492196\end{array}$

8_8_dtu_PBE

$\begin{array}{llll}\text { C } & 1.798467 & 0.614414 & 0.085039 \\ \text { C } & 3.094375 & -0.188556 & -0.102893 \\ \text { C } & 0.549824 & -0.160662 & -0.254526 \\ \text { C } & -0.660251 & -0.124206 & 0.475669 \\ \text { C } & -1.961694 & -0.676222 & 0.039379 \\ \text { H } & 1.789472 & 1.477121 & -0.610281 \\ \text { H } & 3.155643 & -1.028783 & 0.607071 \\ \text { H } & 3.179359 & -0.590369 & -1.124937 \\ \text { H } & 3.962717 & 0.461949 & 0.073528 \\ \text { H } & 0.507564 & -0.64574 & -1.243075 \\ \text { H } & 0.410085 & -1.105503 & 0.535641 \\ \text { H } & -0.621149 & 0.369958 & 1.460742 \\ \text { H } & -1.862523 & -1.28679 & -0.871803 \\ \text { H } & 1.723462 & 1.034103 & 1.102761 \\ \mathrm{C} & -2.891534 & 0.550673 & -0.224317 \\ \text { H } & -3.874281 & 0.159343 & -0.526638 \\ \text { H } & -3.031998 & 1.163163 & 0.678993 \\ \text { H } & -2.505085 & 1.185163 & -1.035055 \\ \text { H } & -2.408385 & -1.286266 & 0.842947\end{array}$




\begin{tabular}{|c|c|c|c|}
\hline \multicolumn{4}{|c|}{$1,3 \mathrm{H}$} \\
\hline \multicolumn{4}{|c|}{ 3_7C_PBE } \\
\hline C & -0.220134 & -0.529332 & 1.062718 \\
\hline C & 0.679042 & 0.06453 & -0.080846 \\
\hline $\mathrm{H}$ & 0.018392 & -0.001568 & -1.049575 \\
\hline$C$ & 0.93028 & 1.568315 & 0.062862 \\
\hline $\mathrm{H}$ & 1.432795 & 1.963159 & -0.831935 \\
\hline $\mathrm{H}$ & 0.004379 & 2.140111 & 0.226221 \\
\hline $\mathrm{H}$ & 1.583428 & 1.726152 & 0.935758 \\
\hline $\mathrm{C}$ & -1.085312 & -0.662964 & -0.103471 \\
\hline $\mathrm{H}$ & -1.012347 & -1.604145 & -0.668032 \\
\hline $\mathrm{H}$ & -0.524304 & 0.212561 & 1.80933 \\
\hline $\mathrm{H}$ & 0.18509 & -1.449694 & 1.495747 \\
\hline C & 1.87433 & -0.830197 & -0.424643 \\
\hline $\mathrm{H}$ & 2.380412 & -0.473652 & -1.333419 \\
\hline $\mathrm{H}$ & 2.586205 & -0.79186 & 0.415341 \\
\hline $\mathrm{H}$ & 1.580148 & -1.880562 & -0.573343 \\
\hline $\mathrm{C}$ & -2.254152 & 0.21515 & -0.379016 \\
\hline $\mathrm{H}$ & -2.491155 & 0.308405 & -1.448508 \\
\hline $\mathrm{H}$ & -3.109982 & -0.304944 & 0.0989 \\
\hline $\mathrm{H}$ & -2.177381 & 1.203032 & 0.097887 \\
\hline \multicolumn{4}{|c|}{ 3_7C_CC } \\
\hline $\mathrm{C}$ & -0.214133 & -0.534533 & 1.06492 \\
\hline $\mathrm{C}$ & 0.658901 & 0.063567 & -0.081407 \\
\hline $\mathrm{H}$ & -0.002736 & -0.001983 & -1.028686 \\
\hline C & 0.918657 & 1.561734 & 0.070094 \\
\hline $\mathrm{H}$ & 1.399047 & 1.953124 & -0.824422 \\
\hline $\mathrm{H}$ & 0.00267 & 2.121466 & 0.250701 \\
\hline $\mathrm{H}$ & 1.5832 & 1.7075 & 0.921057 \\
\hline C & -1.062976 & -0.666039 & -0.100933 \\
\hline $\mathrm{H}$ & -0.991526 & -1.595752 & -0.658738 \\
\hline $\mathrm{H}$ & -0.523381 & 0.195791 & 1.802863 \\
\hline $\mathrm{H}$ & 0.196636 & -1.44401 & 1.484163 \\
\hline $\mathrm{C}$ & 1.856656 & -0.818854 & -0.431323 \\
\hline $\mathrm{H}$ & 2.344354 & -0.459006 & -1.335129 \\
\hline $\mathrm{H}$ & 2.567006 & -0.778166 & 0.393864 \\
\hline $\mathrm{H}$ & 1.560099 & -1.856919 & -0.579693 \\
\hline $\mathrm{C}$ & -2.235693 & 0.217546 & -0.380132 \\
\hline $\mathrm{H}$ & -2.434501 & 0.324869 & -1.443758 \\
\hline $\mathrm{H}$ & -3.08784 & -0.297599 & 0.074416 \\
\hline $\mathrm{H}$ & -2.141498 & 1.190159 & 0.096047 \\
\hline
\end{tabular}




$\begin{array}{llll}\text { 5CA_5CA_duu_PBE } & & \\ & & & \\ \mathrm{C} & 0.044865 & 0.678268 & 0.456307 \\ \mathrm{C} & 0.478784 & -0.777055 & 0.459918 \\ \mathrm{H} & -0.419777 & -1.258101 & -0.346617 \\ \mathrm{C} & 0.951549 & 1.752475 & -0.123619 \\ \mathrm{H} & 1.350384 & 1.486706 & -1.113935 \\ \mathrm{H} & 0.388902 & 2.692269 & -0.227533 \\ \mathrm{H} & 1.798309 & 1.945058 & 0.552205 \\ \mathrm{C} & -0.920551 & -0.058916 & -0.443165 \\ \mathrm{H} & -0.694192 & 0.049847 & -1.515249 \\ \mathrm{H} & -0.421739 & 0.97059 & 1.405907 \\ \mathrm{C} & -2.357057 & -0.286053 & -0.03292 \\ \mathrm{H} & -2.891356 & 0.658684 & -0.235314 \\ \mathrm{H} & -2.843359 & -1.085313 & -0.609797 \\ \mathrm{H} & -2.451097 & -0.493743 & 1.043707 \\ \mathrm{H} & 0.12996 & -1.338422 & 1.339316 \\ \mathrm{C} & 1.759404 & -1.240309 & -0.197411 \\ \mathrm{H} & 2.579984 & -0.959215 & 0.48539 \\ \mathrm{H} & 1.78253 & -2.330766 & -0.33132 \\ \mathrm{H} & 1.949483 & -0.748053 & -1.161415 \\ & & & \\ \text { 5CA_5CA_duu_CC } & & \\ & & & \\ \mathrm{C} & 0.041353 & 0.681481 & 0.450629 \\ \mathrm{C} & 0.43512 & -0.789473 & 0.447267 \\ \mathrm{H} & -0.407877 & -1.255744 & -0.316452 \\ \mathrm{C} & 0.992132 & 1.718883 & -0.118806 \\ \mathrm{H} & 1.360157 & 1.442973 & -1.105234 \\ \mathrm{H} & 0.47357 & 2.672462 & -0.203407 \\ \mathrm{H} & 1.84342 & 1.855612 & 0.546111 \\ \mathrm{C} & -0.915537 & -0.018027 & -0.442686 \\ \mathrm{H} & -0.690008 & 0.052373 & -1.504551 \\ \mathrm{H} & -0.408048 & 0.977671 & 1.393133 \\ \mathrm{C} & -2.347647 & -0.262988 & -0.034842 \\ \mathrm{H} & -2.888002 & 0.662481 & -0.241133 \\ \mathrm{H} & -2.80712 & -1.068378 & -0.602983 \\ \mathrm{H} & -2.423065 & -0.46913 & 1.031456 \\ \mathrm{C} & 1.735284 & -1.246218 & -0.191353 \\ \mathrm{H} & 2.552273 & -0.898006 & 0.440071 \\ \mathrm{H} & 1.775298 & -2.331079 & -0.252361 \\ & 1.868587 & -0.827569 & -1.185999 \\ 0.106593 & -1.315616 & 1.340095\end{array}$




$\begin{array}{llll}\text { 5CA_5CA_udu_PBE } & & \\ & & & \\ \text { C } & 0.736129 & 0.000003 & -0.372137 \\ \text { C } & -0.156604 & 0.909999 & 0.442079 \\ \text { H } & -0.808263 & -0.000004 & 1.113085 \\ \text { C } & 2.234154 & 0.000008 & -0.114159 \\ \text { H } & 2.464855 & -0.000002 & 0.963333 \\ \text { H } & 2.700042 & -0.888959 & -0.565172 \\ \text { H } & 2.700035 & 0.888986 & -0.565156 \\ \text { C } & -0.156597 & -0.910005 & 0.44207 \\ \text { H } & 0.336038 & -1.325174 & 1.33532 \\ \text { C } & -1.256485 & 1.724149 & -0.19487 \\ \text { H } & -0.767905 & 2.613538 & -0.630136 \\ \text { H } & -1.755422 & 1.191466 & -1.017661 \\ \text { H } & -2.004391 & 2.068316 & 0.533277 \\ \text { H } & 0.461902 & 0.000008 & -1.436196 \\ \text { C } & -1.256473 & -1.724154 & -0.194888 \\ \text { H } & -0.767888 & -2.613547 & -0.63014 \\ \text { H } & -2.004392 & -2.068314 & 0.533248 \\ \text { H } & -1.755394 & -1.191475 & -1.017693 \\ \text { H } & 0.336035 & 1.325164 & 1.335329 \\ & & & \\ \text { 5CA_5CA_udu_CC } & & \\ & & & \\ \text { C } & -0.730564 & -0.000121 & -0.374957 \\ \text { C } & 0.151592 & -0.897099 & 0.442995 \\ \text { H } & 0.802405 & 0.000218 & 1.088672 \\ \text { C } & -2.225067 & -0.000181 & -0.11374 \\ \text { H } & -2.43733 & -0.00034 & 0.95573 \\ \text { H } & -2.683584 & 0.882123 & -0.557312 \\ \text { H } & -2.68356 & -0.882368 & -0.557569 \\ \text { C } & 0.151514 & 0.896988 & 0.443147 \\ \text { H } & -0.334216 & 1.312117 & 1.323648 \\ \text { C } & 1.252708 & -1.714345 & -0.19556 \\ \text { H } & 0.772971 & -2.593785 & -0.626982 \\ \text { H } & 1.744064 & -1.170909 & -0.999344 \\ \text { H } & 1.990893 & -2.042393 & 0.532441 \\ \text { H } & -0.458811 & 0 & -1.426746 \\ \text { C } & 1.252262 & 1.714653 & -0.195577 \\ \text { H } & 0.77217 & 2.593795 & -0.627198 \\ \text { H } & 1.990288 & 2.043146 & 0.532384 \\ \text { H } & 1.743903 & 1.171267 & -0.999223 \\ & -0.333858 & -1.312247 & 1.323641\end{array}$




$\begin{array}{llll}\text { 5CA_5CA_uuu_PBE } & & \\ & & & \\ \text { C } & 0.000045 & 0.766669 & 0.687934 \\ \text { C } & -0.91002 & -0.448044 & 0.629557 \\ \text { H } & -0.000136 & -1.381161 & 0.6358 \\ \text { C } & 0.910173 & -0.447842 & 0.629522 \\ \text { H } & 1.325533 & -0.738929 & 1.605745 \\ \text { C } & -1.729551 & -0.789392 & -0.592203 \\ \text { H } & -2.587276 & -0.09433 & -0.597299 \\ \text { H } & -1.185866 & -0.642457 & -1.535605 \\ \text { H } & -2.119628 & -1.815837 & -0.550832 \\ \text { C } & 1.729509 & -0.789391 & -0.592263 \\ \text { H } & 2.587329 & -0.094437 & -0.597412 \\ \text { H } & 2.119473 & -1.81588 & -0.550902 \\ \text { H } & 1.185788 & -0.642362 & -1.535628 \\ \text { H } & -1.32546 & -0.738878 & 1.605822 \\ \text { C } & -0.000068 & 1.792275 & -0.434193 \\ \text { H } & -0.887038 & 2.437521 & -0.345068 \\ \text { H } & 0.886673 & 2.437818 & -0.344922 \\ \text { H } & 0.000097 & 1.350864 & -1.44031 \\ \text { H } & -0.000015 & 1.232417 & 1.680482 \\ & & & \\ \text { 5CA_5CA_uuu_CC } & & \\ & & & \\ \text { C } & 0 & 0.760718 & 0.68992 \\ \text { C } & -0.897604 & -0.446834 & 0.629831 \\ \text { H } & 0 & -1.36149 & 0.608554 \\ \text { C } & 0.897605 & -0.446833 & 0.62983 \\ \text { H } & 1.309091 & -0.740943 & 1.591838 \\ \text { C } & -1.725142 & -0.780584 & -0.593039 \\ \text { H } & -2.562565 & -0.082017 & -0.605596 \\ \text { H } & -1.169855 & -0.658083 & -1.518807 \\ \text { H } & -2.116041 & -1.793656 & -0.53746 \\ \text { C } & 1.725142 & -0.780583 & -0.593039 \\ \text { H } & 2.562566 & -0.082017 & -0.605596 \\ \text { H } & 2.11604 & -1.793655 & -0.537461 \\ \text { H } & 1.169856 & -0.658082 & -1.518807 \\ \text { H } & -1.30909 & -0.740943 & 1.591838 \\ \text { H } & 0 & 1.222518 & 1.670998 \\ \text { C } & -0.000001 & 1.781585 & -0.434125 \\ \text { H } & -0.880518 & 2.416174 & -0.346319 \\ \text { H } & 0.880515 & 2.416176 & -0.346319 \\ \text { H } & -0.000001 & 1.331203 & -1.423132\end{array}$




$\begin{array}{llll}\text { 9C_8CA_gd_PBE } & & \\ \text { C } & -0.502183 & 1.12743 & -0.495591 \\ \text { C } & -1.509879 & 0.351476 & 0.309773 \\ \text { H } & -0.679233 & -0.180515 & 1.176517 \\ \text { C } & 0.299828 & 0.41527 & 0.564742 \\ \text { H } & 0.572822 & 1.049273 & 1.422733 \\ \text { C } & -2.213204 & -0.852942 & -0.266891 \\ \text { H } & -3.038835 & -0.457685 & -0.884677 \\ \text { H } & -1.5629 & -1.44383 & -0.928298 \\ \text { H } & -2.646403 & -1.500516 & 0.50811 \\ \text { H } & -0.345524 & 0.760455 & -1.515968 \\ \text { C } & 1.240326 & -0.72102 & 0.207345 \\ \text { H } & 1.411013 & -1.364591 & 1.085209 \\ \text { H } & 0.799705 & -1.343299 & -0.589246 \\ \text { H } & -2.050479 & 0.948414 & 1.059362 \\ \text { H } & -0.553226 & 2.216954 & -0.421818 \\ \text { C } & 2.574449 & -0.113386 & -0.27341 \\ \text { H } & 3.049664 & 0.498345 & 0.509009 \\ \text { H } & 3.266028 & -0.931461 & -0.523984 \\ \text { H } & 2.441344 & 0.507489 & -1.172764 \\ & & & \\ \text { 9C_8CA_gd_CC } & & \\ & & & \\ \text { C } & -0.486103 & 1.117791 & -0.486615 \\ \text { C } & -1.510059 & 0.376786 & 0.270055 \\ \text { H } & -0.62632 & -0.19384 & 1.185723 \\ \text { C } & 0.266718 & 0.383796 & 0.615396 \\ \text { H } & 0.556669 & 1.041967 & 1.43168 \\ \text { C } & -2.1863 & -0.850725 & -0.280064 \\ \text { H } & -3.024707 & -0.482924 & -0.875442 \\ \text { H } & -1.529217 & -1.415715 & -0.936761 \\ \text { H } & -2.577243 & -1.492962 & 0.505415 \\ \text { H } & -0.283885 & 0.749095 & -1.484932 \\ \text { C } & 1.241884 & -0.722991 & 0.228933 \\ \text { H } & 1.446878 & -1.3441 & 1.100938 \\ \text { H } & 0.789649 & -1.360501 & -0.532084 \\ \text { H } & -2.038257 & 0.940194 & 1.034401 \\ \text { H } & -0.498389 & 2.195727 & -0.404774 \\ \text { C } & 2.5377 & -0.100474 & -0.30121 \\ \text { H } & 3.007191 & 0.532541 & 0.451442 \\ \text { H } & 3.23859 & -0.891697 & -0.560127 \\ \text { H } & 2.355998 & 0.497122 & -1.194456\end{array}$




\begin{tabular}{|c|c|c|c|}
\hline \multicolumn{4}{|c|}{ 9C_8CA_gg_PBE } \\
\hline C & -0.656419 & -0.991527 & 0.842018 \\
\hline C & -1.40443 & -0.263208 & -0.236362 \\
\hline $\mathrm{H}$ & -0.438702 & -0.30838 & -1.159393 \\
\hline$C$ & 0.272584 & -1.011195 & -0.351337 \\
\hline $\mathrm{H}$ & 0.207415 & -1.946863 & -0.927188 \\
\hline C & -1.725095 & 1.207806 & -0.144193 \\
\hline $\mathrm{H}$ & -2.673354 & 1.260633 & 0.421651 \\
\hline $\mathrm{H}$ & -0.970592 & 1.77568 & 0.414904 \\
\hline $\mathrm{H}$ & -1.897724 & 1.668327 & -1.127182 \\
\hline $\mathrm{H}$ & -0.327353 & -0.365731 & 1.678355 \\
\hline C & 1.635314 & -0.328287 & -0.368464 \\
\hline $\mathrm{H}$ & 2.001741 & -0.300889 & -1.40754 \\
\hline $\mathrm{H}$ & -2.108956 & -0.889079 & -0.804031 \\
\hline $\mathrm{H}$ & -1.058226 & -1.959925 & 1.151781 \\
\hline $\mathrm{H}$ & 2.268849 & -1.071475 & 0.156785 \\
\hline C & 1.785396 & 1.042708 & 0.294734 \\
\hline $\mathrm{H}$ & 1.26744 & 1.833786 & -0.269272 \\
\hline $\mathrm{H}$ & 1.433515 & 1.054746 & 1.338509 \\
\hline $\mathrm{H}$ & 2.851843 & 1.311393 & 0.314246 \\
\hline \multicolumn{4}{|c|}{ 9C_8CA_gg_CC } \\
\hline C & -0.673976 & -1.01221 & 0.820465 \\
\hline C & -1.377537 & -0.255987 & -0.254935 \\
\hline $\mathrm{H}$ & -0.406001 & -0.279785 & -1.1385 \\
\hline C & 0.26423 & -1.009382 & -0.354624 \\
\hline $\mathrm{H}$ & 0.202811 & -1.920676 & -0.944826 \\
\hline $\mathrm{C}$ & -1.682645 & 1.219182 & -0.126984 \\
\hline $\mathrm{H}$ & -2.643005 & 1.271297 & 0.389469 \\
\hline $\mathrm{H}$ & -0.942964 & 1.738085 & 0.473615 \\
\hline $\mathrm{H}$ & -1.787785 & 1.702688 & -1.095342 \\
\hline $\mathrm{H}$ & -0.359757 & -0.416745 & 1.668175 \\
\hline $\mathrm{C}$ & 1.636792 & -0.344359 & -0.328814 \\
\hline $\mathrm{H}$ & 2.03279 & -0.34352 & -1.344508 \\
\hline $\mathrm{H}$ & -2.07276 & -0.846305 & -0.845878 \\
\hline $\mathrm{H}$ & -1.089211 & -1.973937 & 1.087813 \\
\hline $\mathrm{H}$ & 2.244024 & -1.050764 & 0.245046 \\
\hline$C$ & 1.741173 & 1.052031 & 0.280363 \\
\hline $\mathrm{H}$ & 1.22874 & 1.796433 & -0.328073 \\
\hline $\mathrm{H}$ & 1.353735 & 1.092818 & 1.298074 \\
\hline $\mathrm{H}$ & 2.79116 & 1.334762 & 0.322114 \\
\hline
\end{tabular}




$\begin{array}{llll}\text { 9C_8CA_gt_PBE } & & \\ \text { C } & -1.053395 & -1.238526 & 0.283619 \\ \text { C } & -1.419597 & -0.0109 & -0.475524 \\ \text { H } & -0.144281 & 0.247488 & -0.964917 \\ \text { C } & 0.251594 & -0.7527 & -0.336598 \\ \text { H } & 0.547459 & -1.330646 & -1.226237 \\ \text { C } & -1.763698 & 1.28205 & 0.208754 \\ \text { H } & -2.835343 & 1.203074 & 0.469093 \\ \text { H } & -1.211862 & 1.424721 & 1.14928 \\ \text { H } & -1.639635 & 2.158097 & -0.443182 \\ \text { H } & -1.095847 & -1.148138 & 1.374761 \\ \text { C } & 1.36158 & -0.198007 & 0.543069 \\ \text { H } & -1.834033 & -0.182666 & -1.479937 \\ \text { H } & -1.436278 & -2.189784 & -0.095726 \\ \text { H } & 1.804901 & -1.095067 & 1.016362 \\ \text { C } & 2.42401 & 0.600698 & -0.21616 \\ \text { H } & 3.231876 & 0.899614 & 0.467336 \\ \text { H } & 2.876929 & 0.006921 & -1.025893 \\ \text { H } & 2.006384 & 1.522548 & -0.655146 \\ \text { H } & 0.926765 & 0.388157 & 1.371244 \\ & & & \\ \text { 9C_8CA_gt_CC } & & \\ & & & \\ \text { C } & -1.050707 & -1.228885 & 0.289752 \\ \text { C } & -1.428672 & -0.026741 & -0.463871 \\ \text { H } & -0.120641 & 0.239736 & -0.957034 \\ \text { C } & 0.242103 & -0.727374 & -0.353292 \\ \text { H } & 0.537566 & -1.33015 & -1.209523 \\ \text { C } & -1.747972 & 1.281518 & 0.205179 \\ \text { H } & -2.81044 & 1.230652 & 0.454111 \\ \text { H } & -1.192286 & 1.411013 & 1.130781 \\ \text { H } & -1.589934 & 2.131888 & -0.453773 \\ \text { H } & -1.070963 & -1.134841 & 1.368688 \\ \text { C } & 1.352202 & -0.196909 & 0.546409 \\ \text { H } & -1.827466 & -0.191048 & -1.461065 \\ \text { H } & -1.411886 & -2.177756 & -0.081852 \\ \text { H } & 1.782639 & -1.079295 & 1.025716 \\ \text { C } & 2.418047 & 0.586825 & -0.215925 \\ \text { H } & 3.213715 & 0.890075 & 0.46157 \\ \text { H } & 2.862197 & -0.01791 & -1.006624 \\ \text { H } & 2.000627 & 1.490214 & -0.665015 \\ \text { H } & 0.916855 & 0.406822 & 1.344493\end{array}$




$\begin{array}{llll}\text { 9C_8CA_td_PBE_max } & & \\ \text { C } & 0.46907 & 1.067097 & 0.189492 \\ \mathrm{C} & 1.377939 & -0.100723 & 0.464667 \\ \mathrm{H} & 0.630489 & -1.045519 & -0.047099 \\ \mathrm{C} & -0.242977 & -0.139405 & -0.365286 \\ \mathrm{H} & -0.132557 & -0.266759 & -1.453431 \\ \mathrm{H} & -0.003721 & 1.526461 & 1.063335 \\ \mathrm{C} & -1.510951 & -0.659939 & 0.290155 \\ \mathrm{H} & -1.659905 & -1.723452 & 0.043398 \\ \mathrm{H} & -1.424209 & -0.574869 & 1.386939 \\ \mathrm{H} & 0.843756 & 1.789302 & -0.542343 \\ \mathrm{C} & -2.702536 & 0.176102 & -0.218148 \\ \mathrm{H} & -2.823917 & 0.091908 & -1.309235 \\ \mathrm{H} & -3.624092 & -0.200163 & 0.250425 \\ \mathrm{H} & -2.598313 & 1.240422 & 0.043419 \\ \mathrm{H} & 1.317749 & -0.488818 & 1.492443 \\ \mathrm{C} & 2.677559 & -0.26334 & -0.286921 \\ \mathrm{H} & 3.406558 & 0.403581 & 0.206117 \\ \mathrm{H} & 3.067509 & -1.289812 & -0.24207 \\ \mathrm{H} & 2.592027 & 0.05897 & -1.335656 \\ & & & \\ \text { 9C_8CA_td_CC } & & \\ & & & \\ \mathrm{C} & -0.449662 & 1.054184 & -0.160115 \\ \mathrm{C} & -1.371403 & -0.041192 & -0.486495 \\ \mathrm{H} & -0.575585 & -1.067609 & 0.071047 \\ \mathrm{C} & 0.206629 & -0.21626 & 0.376539 \\ \mathrm{H} & 0.116214 & -0.303195 & 1.457426 \\ \mathrm{H} & 0.079377 & 1.508926 & -0.987481 \\ \mathrm{C} & 1.508118 & -0.674728 & -0.276187 \\ \mathrm{H} & 1.692701 & -1.717914 & -0.01948 \\ \mathrm{H} & 1.399998 & -0.615958 & -1.361502 \\ \mathrm{H} & -0.788416 & 1.754084 & 0.592437 \\ \mathrm{C} & 2.667958 & 0.206262 & 0.196139 \\ \mathrm{H} & 2.790608 & 0.147889 & 1.27745 \\ \mathrm{H} & 3.592409 & -0.134416 & -0.266144 \\ \mathrm{H} & 2.515941 & 1.249429 & -0.080933 \\ \mathrm{H} & -1.291077 & -0.453008 & -1.488831 \\ \mathrm{H} & -2.656485 & -0.251641 & 0.267157 \\ \mathrm{H} & -3.393652 & 0.397761 & -0.209947 \\ & -3.009082 & -1.278315 & 0.205877 \\ & -2.560365 & 0.052579 & 1.307851\end{array}$




$\begin{array}{llll}\text { 9C_8CA_tt_PBE_max } & & \\ \text { C } & -0.871974 & 1.252557 & 0.055788 \\ \mathrm{C} & -1.267917 & -0.072166 & -0.492837 \\ \mathrm{H} & -0.154744 & -0.767171 & -0.035568 \\ \mathrm{C} & 0.24532 & 0.298736 & 0.461493 \\ \mathrm{H} & 0.176322 & -0.024976 & 1.511741 \\ \mathrm{H} & -0.575065 & 2.015512 & -0.67047 \\ \mathrm{C} & 1.628935 & 0.451804 & -0.156247 \\ \mathrm{H} & -1.488807 & 1.635257 & 0.874444 \\ \mathrm{H} & -1.012162 & -0.239425 & -1.549492 \\ \mathrm{C} & -2.391855 & -0.875028 & 0.101632 \\ \mathrm{H} & -3.317178 & -0.482011 & -0.357779 \\ \mathrm{H} & -2.326661 & -1.946142 & -0.134763 \\ \mathrm{H} & -2.478775 & -0.729779 & 1.18865 \\ \mathrm{H} & 2.058679 & 1.335585 & 0.352395 \\ \mathrm{H} & 1.525025 & 0.73767 & -1.217724 \\ \mathrm{C} & 2.534344 & -0.770321 & 0.01173 \\ \mathrm{H} & 3.533506 & -0.557399 & -0.394536 \\ \mathrm{H} & 2.657931 & -1.042538 & 1.071705 \\ \mathrm{H} & 2.140816 & -1.648076 & -0.527963 \\ & & & \\ \text { 9C_8CA_tt_CC } & & \\ & & & \\ \mathrm{C} & -0.868892 & 1.242672 & 0.0789 \\ \mathrm{C} & -1.275995 & -0.04043 & -0.500964 \\ \mathrm{H} & -0.135393 & -0.767939 & -0.042424 \\ \mathrm{C} & 0.234374 & 0.250681 & 0.452517 \\ \mathrm{H} & 0.177025 & -0.056677 & 1.49487 \\ \mathrm{H} & -0.549568 & 2.013013 & -0.610925 \\ \mathrm{C} & 1.614447 & 0.452786 & -0.164384 \\ \mathrm{H} & -1.463859 & 1.60257 & 0.908054 \\ \mathrm{H} & -1.018703 & -0.200435 & -1.544551 \\ \mathrm{C} & -2.378278 & -0.871979 & 0.094514 \\ \mathrm{H} & -3.306764 & -0.488286 & -0.334205 \\ \mathrm{H} & -2.288286 & -1.924624 & -0.162297 \\ \mathrm{H} & -2.428466 & -0.749656 & 1.174922 \\ \mathrm{H} & 2.02909 & 1.338971 & 0.321414 \\ \mathrm{H} & 1.49441 & 0.702638 & -1.221233 \\ \mathrm{C} & 2.535365 & -0.751627 & 0.014296 \\ \mathrm{H} & 3.517346 & -0.534048 & -0.400939 \\ \mathrm{H} & 2.663152 & -0.997003 & 1.06862 \\ & 2.143898 & -1.631137 & -0.500582\end{array}$




\section{$1,2 \mathrm{R}$}

5CA_5CA_dud_PBE

$\begin{array}{llll}\mathrm{C} & -0.348318 & -0.703317 & -0.50885 \\ \mathrm{C} & -0.348321 & 0.703317 & -0.50885 \\ \mathrm{C} & 0.977852 & -0.000001 & 0.570665 \\ \mathrm{C} & -1.360468 & -1.581306 & 0.16033 \\ \mathrm{C} & -1.360472 & 1.581304 & 0.160331 \\ \mathrm{C} & 2.358129 & 0.000002 & -0.06123 \\ \mathrm{H} & 0.783364 & -0.863635 & 1.229924 \\ \mathrm{H} & -2.132561 & -1.811208 & -0.596836 \\ \mathrm{H} & -0.922906 & -2.541634 & 0.471396 \\ \mathrm{H} & -1.863665 & -1.108407 & 1.014996 \\ \mathrm{H} & -0.922911 & 2.541632 & 0.471399 \\ \mathrm{H} & -2.132563 & 1.811207 & -0.596836 \\ \mathrm{H} & -1.86367 & 1.108403 & 1.014995 \\ \mathrm{H} & 3.103199 & 0 & 0.751416 \\ \mathrm{H} & 2.542439 & -0.895315 & -0.672649 \\ \mathrm{H} & 2.542438 & 0.895323 & -0.672642 \\ \mathrm{H} & 0.286532 & -1.190179 & -1.25973 \\ \mathrm{H} & 0.28653 & 1.190182 & -1.259728 \\ \mathrm{H} & 0.78336 & 0.863631 & 1.229925\end{array}$

5CA_5CA_dud_CC

$\begin{array}{llll}\text { C } & -0.342156 & -0.694961 & -0.519337 \\ \text { C } & -0.342027 & 0.694977 & -0.519329 \\ \text { C } & 0.962018 & -0.000169 & 0.57956 \\ \text { C } & -1.352556 & -1.571638 & 0.162057 \\ \text { C } & -1.352235 & 1.571853 & 0.162087 \\ \text { C } & 2.340565 & -0.000062 & -0.059493 \\ \text { H } & 0.776466 & -0.861858 & 1.220411 \\ \text { H } & -2.142431 & -1.774031 & -0.564456 \\ \text { H } & -0.909493 & -2.524027 & 0.446311 \\ \text { H } & -1.801565 & -1.104164 & 1.034169 \\ \text { H } & -0.908945 & 2.524109 & 0.446434 \\ \text { H } & -2.142018 & 1.774503 & -0.564456 \\ \text { H } & -1.80141 & 1.104425 & 1.034137 \\ \text { H } & 3.079785 & -0.000367 & 0.741496 \\ \text { H } & 2.508607 & -0.886377 & -0.66792 \\ \text { H } & 2.50873 & 0.886609 & -0.667365 \\ \text { H } & 0.28698 & -1.181459 & -1.256399 \\ \text { H } & 0.287219 & 1.181365 & -1.256368 \\ \text { H } & 0.776426 & 0.861265 & 1.220738\end{array}$




\begin{tabular}{|c|c|c|c|}
\hline \multicolumn{4}{|c|}{ 5CA_5CA_duu_PBE } \\
\hline C & -0.938194 & 0.083412 & -0.428834 \\
\hline C & -0.048316 & 0.805277 & 0.390496 \\
\hline $\mathrm{C}$ & 0.399683 & -0.918968 & 0.48004 \\
\hline $\mathrm{C}$ & -2.344057 & -0.256878 & -0.065314 \\
\hline $\mathrm{C}$ & 1.6973 & -1.244999 & -0.236394 \\
\hline $\mathrm{H}$ & -0.366846 & -1.692891 & 0.28278 \\
\hline $\mathrm{H}$ & -2.976235 & 0.517378 & -0.541996 \\
\hline $\mathrm{H}$ & -2.668909 & -1.225131 & -0.4749 \\
\hline $\mathrm{H}$ & -2.530359 & -0.213805 & 1.018034 \\
\hline $\mathrm{H}$ & 1.990122 & -2.266746 & 0.055585 \\
\hline $\mathrm{H}$ & 1.591527 & -1.23825 & -1.331447 \\
\hline $\mathrm{H}$ & 2.515163 & -0.569574 & 0.048186 \\
\hline $\mathrm{H}$ & 0.44649 & -0.905955 & 1.581577 \\
\hline $\mathrm{C}$ & 1.073495 & 1.670475 & -0.114665 \\
\hline $\mathrm{H}$ & 1.421304 & 1.385993 & -1.117399 \\
\hline $\mathrm{H}$ & 0.681699 & 2.701047 & -0.167789 \\
\hline $\mathrm{H}$ & 1.923001 & 1.685806 & 0.584288 \\
\hline $\mathrm{H}$ & -0.432669 & 1.074211 & 1.383567 \\
\hline $\mathrm{H}$ & -0.633757 & -0.081996 & -1.472461 \\
\hline \multicolumn{4}{|c|}{ 5CA_5CA_duu_CC } \\
\hline $\mathrm{C}$ & -0.908874 & 0.103099 & -0.432772 \\
\hline $\mathrm{C}$ & -0.047432 & 0.831621 & 0.379128 \\
\hline $\mathrm{C}$ & 0.362213 & -0.918865 & 0.497982 \\
\hline $\mathrm{C}$ & -2.323785 & -0.229483 & -0.072281 \\
\hline $\mathrm{C}$ & 1.638967 & -1.283961 & -0.239777 \\
\hline $\mathrm{H}$ & -0.40727 & -1.679158 & 0.35549 \\
\hline $\mathrm{H}$ & -2.951886 & 0.528127 & -0.547205 \\
\hline $\mathrm{H}$ & -2.623856 & -1.200974 & -0.460132 \\
\hline $\mathrm{H}$ & -2.489404 & -0.186274 & 1.002618 \\
\hline $\mathrm{H}$ & 1.913897 & -2.291784 & 0.071155 \\
\hline $\mathrm{H}$ & 1.500735 & -1.296398 & -1.319572 \\
\hline $\mathrm{H}$ & 2.46325 & -0.621401 & 0.007179 \\
\hline $\mathrm{H}$ & 0.461491 & -0.842175 & 1.580176 \\
\hline C & 1.113644 & 1.649404 & -0.114639 \\
\hline $\mathrm{H}$ & 1.46149 & 1.328918 & -1.093189 \\
\hline $\mathrm{H}$ & 0.758473 & 2.678253 & -0.193605 \\
\hline $\mathrm{H}$ & 1.938345 & 1.637968 & 0.595271 \\
\hline $\mathrm{H}$ & -0.433662 & 1.10951 & 1.3547 \\
\hline $\mathrm{H}$ & -0.600007 & -0.075501 & -1.458732 \\
\hline
\end{tabular}




$\begin{array}{llll}\text { 5CA_5CA_uuu_PBE } & & \\ & & & \\ \text { C } & -0.769894 & 0.516193 & 0.703957 \\ \text { C } & -0.769894 & 0.516193 & -0.703957 \\ \text { C } & 0.920712 & 0.870906 & 0 \\ \text { C } & 1.890501 & -0.29023 & 0 \\ \text { H } & 1.041341 & 1.548654 & 0.862398 \\ \text { H } & 2.905807 & 0.140603 & 0 \\ \text { H } & 1.807554 & -0.920421 & 0.896548 \\ \text { H } & 1.807554 & -0.920421 & -0.896548 \\ \text { H } & 1.041341 & 1.548654 & -0.862398 \\ \text { H } & -1.061289 & 1.459606 & -1.182343 \\ \text { H } & -1.061289 & 1.459606 & 1.182343 \\ \text { C } & -0.769894 & -0.678773 & -1.601801 \\ \text { H } & -0.159861 & -0.509202 & -2.502594 \\ \text { H } & -0.463274 & -1.611797 & -1.112246 \\ \text { H } & -1.812289 & -0.803688 & -1.948803 \\ \text { C } & -0.769894 & -0.678773 & 1.601801 \\ \text { H } & -0.159861 & -0.509202 & 2.502594 \\ \text { H } & -1.812289 & -0.803688 & 1.948803 \\ \text { H } & -0.463274 & -1.611797 & 1.112246 \\ & & & \\ \text { 5CA_5CA_uuu_CC } & & \\ & & & \\ \text { C } & -0.766833 & 0.523843 & 0.695384 \\ \text { C } & -0.766833 & 0.523843 & -0.695384 \\ \text { C } & 0.919017 & 0.86820 & \\ \text { C } & 1.874198 & -0.309694 & 0 \\ \text { H } & 1.050024 & 1.524609 & 0.85995 \\ \text { H } & 2.881616 & 0.107519 & 0 \\ \text { H } & 1.775192 & -0.929499 & 0.887156 \\ \text { H } & 1.775192 & -0.929499 & -0.887156 \\ \text { H } & 1.050024 & 1.524609 & -0.85995 \\ \text { C } & -0.766833 & -0.673451 & -1.596799 \\ \text { H } & -0.464739 & -1.589848 & -1.100742 \\ \text { H } & -1.794368 & -0.79267 & -1.947324 \\ \text { H } & -0.141102 & -0.502807 & -2.471482 \\ \text { H } & -1.043466 & 1.458584 & -1.170374 \\ \text { C } & -0.766833 & -0.673451 & 1.596799 \\ \text { H } & -1.794368 & -0.79267 & 1.947324 \\ \text { H } & -0.464739 & -1.589848 & 1.100742 \\ \text { H } & -0.141102 & -0.502807 & 2.471482 \\ & -1.043466 & 1.458584 & 1.170374\end{array}$




\begin{tabular}{|c|c|c|c|}
\hline \multicolumn{4}{|c|}{ 6CA_5CB_gd_PBE } \\
\hline C & -1.305283 & -1.305859 & -0.255635 \\
\hline C & -1.040414 & 0.274744 & 0.59871 \\
\hline C & 0.118577 & -0.524173 & 0.474032 \\
\hline C & 1.232704 & -0.344697 & -0.52372 \\
\hline C & 2.390531 & 0.424075 & 0.151581 \\
\hline C & -1.431634 & 1.424519 & -0.274821 \\
\hline $\mathrm{H}$ & -2.347307 & -1.275554 & 0.105059 \\
\hline $\mathrm{H}$ & -0.948982 & -2.304166 & 0.043133 \\
\hline $\mathrm{H}$ & -1.246026 & -1.131702 & -1.333852 \\
\hline $\mathrm{H}$ & -1.583334 & 0.192236 & 1.549391 \\
\hline $\mathrm{H}$ & 0.37588 & -1.126429 & 1.355495 \\
\hline $\mathrm{H}$ & 0.885905 & 0.19288 & -1.420541 \\
\hline $\mathrm{H}$ & 1.589407 & -1.336372 & -0.848955 \\
\hline $\mathrm{H}$ & 3.216386 & 0.528041 & -0.567442 \\
\hline $\mathrm{H}$ & 2.083471 & 1.433614 & 0.464864 \\
\hline $\mathrm{H}$ & 2.778269 & -0.112818 & 1.031228 \\
\hline $\mathrm{H}$ & -1.13991 & 2.342795 & 0.267815 \\
\hline $\mathrm{H}$ & -0.928833 & 1.43182 & -1.251318 \\
\hline $\mathrm{H}$ & -2.521806 & 1.473995 & -0.415757 \\
\hline \multicolumn{4}{|c|}{ 6CA_5CB_gd_CC } \\
\hline C & -1.315588 & -1.295456 & -0.263055 \\
\hline C & -1.024318 & 0.280177 & 0.605818 \\
\hline C & 0.11144 & -0.521148 & 0.489802 \\
\hline C & 1.225775 & -0.363868 & -0.511966 \\
\hline C & 2.375641 & 0.421134 & 0.139639 \\
\hline C & -1.404158 & 1.42972 & -0.279078 \\
\hline $\mathrm{H}$ & -2.349126 & -1.228382 & 0.073733 \\
\hline $\mathrm{H}$ & -0.967427 & -2.281964 & 0.033825 \\
\hline $\mathrm{H}$ & -1.226626 & -1.114111 & -1.326353 \\
\hline $\mathrm{H}$ & -1.577301 & 0.197418 & 1.53558 \\
\hline $\mathrm{H}$ & 0.348969 & -1.131078 & 1.35552 \\
\hline $\mathrm{H}$ & 0.877169 & 0.146624 & -1.408543 \\
\hline $\mathrm{H}$ & 1.572212 & -1.354645 & -0.808913 \\
\hline $\mathrm{H}$ & 3.196053 & 0.510369 & -0.569436 \\
\hline $\mathrm{H}$ & 2.058713 & 1.424877 & 0.421892 \\
\hline $\mathrm{H}$ & 2.750728 & -0.088352 & 1.026932 \\
\hline $\mathrm{H}$ & -1.110452 & 2.340611 & 0.247203 \\
\hline $\mathrm{H}$ & -0.903671 & 1.409578 & -1.242732 \\
\hline $\mathrm{H}$ & -2.481986 & 1.465704 & -0.425665 \\
\hline
\end{tabular}




$\begin{array}{llll}\text { 6CA_5CB_gg_PBE } & & \\ & & & \\ \text { C } & 0.87421 & -1.139825 & 0.8779 \\ \text { C } & 1.183856 & 0.006017 & -0.505526 \\ \text { C } & 0.011633 & -0.776415 & -0.632131 \\ \text { C } & -1.447047 & -0.43739 & -0.424346 \\ \text { C } & -1.876169 & 0.768462 & 0.414969 \\ \text { C } & 1.33318 & 1.41839 & -0.032483 \\ \text { H } & 1.942136 & -0.985094 & 1.112906 \\ \text { H } & 0.765866 & -2.230313 & 0.795577 \\ \text { H } & 0.258297 & -0.676219 & 1.654424 \\ \text { H } & 2.065265 & -0.405645 & -1.016255 \\ \text { H } & 0.146294 & -1.67296 & -1.25148 \\ \text { H } & -1.940163 & -1.356754 & -0.060632 \\ \text { H } & -1.821375 & -0.322332 & -1.462122 \\ \text { H } & -2.974848 & 0.783247 & 0.469296 \\ \text { H } & -1.513031 & 0.721259 & 1.455005 \\ \text { H } & -1.562361 & 1.724655 & -0.028385 \\ \text { H } & 1.294731 & 2.055144 & -0.936051 \\ \text { H } & 0.542067 & 1.750247 & 0.649045 \\ \text { H } & 2.319136 & 1.579333 & 0.42837 \\ & & & \\ \text { 6CA_5CB_gg_CC } & & \\ & & & \\ \text { C } & 0.905994 & -1.118641 & 0.879201 \\ \text { C } & 1.159892 & 0.006533 & -0.522919 \\ \text { C } & 0.009471 & -0.776108 & -0.64 \\ \text { C } & -1.448955 & -0.466591 & -0.389945 \\ \text { C } & -1.856012 & 0.774418 & 0.401721 \\ \text { C } & 1.304882 & 1.421057 & -0.038134 \\ \text { H } & 1.970498 & -0.991328 & 1.074095 \\ \text { H } & 0.738328 & -2.189204 & 0.803998 \\ \text { H } & 0.316802 & -0.63359 & 1.646072 \\ \text { H } & 2.030418 & -0.388869 & -1.037046 \\ \text { H } & 0.146345 & -1.671875 & -1.237557 \\ \text { H } & -1.891943 & -1.362641 & 0.051104 \\ \text { H } & -1.866795 & -0.4083 & -1.400813 \\ \text { H } & -2.941924 & 0.789866 & 0.474712 \\ \text { H } & -1.468365 & 0.770484 & 1.420592 \\ \text { H } & -1.548204 & 1.693345 & -0.092681 \\ \text { H } & 1.205476 & 2.068988 & -0.911634 \\ \text { H } & 0.558278 & 1.706833 & 0.692888 \\ \text { H } & 2.299458 & 1.572279 & 0.37673\end{array}$




$\begin{array}{llll}\text { 6CA_5CB_gt_PBE } & & \\ & & & \\ \text { C } & 0.472497 & 1.472765 & -0.389596 \\ \text { C } & 1.308389 & 0.086311 & 0.50168 \\ \text { C } & -0.095356 & 0.177737 & 0.646765 \\ \text { C } & -1.10877 & -0.759696 & 0.035733 \\ \text { C } & -2.490689 & -0.131604 & -0.183908 \\ \text { C } & 2.044972 & -0.900591 & -0.343312 \\ \text { H } & 1.399865 & 1.465307 & -0.989468 \\ \text { H } & 0.421632 & 2.394664 & 0.196719 \\ \text { H } & -0.334638 & 1.363273 & -1.129085 \\ \text { H } & 1.913646 & 0.65653 & 1.219724 \\ \text { H } & -0.451797 & 0.731934 & 1.525473 \\ \text { H } & -1.191806 & -1.588514 & 0.76616 \\ \text { H } & -0.724915 & -1.212286 & -0.89354 \\ \text { H } & -3.201081 & -0.898614 & -0.523923 \\ \text { H } & -2.890332 & 0.307148 & 0.744217 \\ \text { H } & -2.471618 & 0.656947 & -0.955052 \\ \text { H } & 2.288499 & -1.749327 & 0.324301 \\ \text { H } & 1.454844 & -1.297394 & -1.180509 \\ \text { H } & 3.001446 & -0.499197 & -0.709186 \\ & & & \\ \text { 6CA_5CB_gt_CC } & & \\ & & & \\ \text { C } & 0.575937 & 1.459125 & -0.39568 \\ \text { C } & 1.273253 & 0.077234 & 0.514769 \\ \text { C } & -0.110754 & 0.148134 & 0.667013 \\ \text { C } & -1.126459 & -0.745567 & 0.010371 \\ \text { C } & -2.492198 & -0.084671 & -0.186737 \\ \text { C } & 2.004922 & -0.923797 & -0.334956 \\ \text { H } & 1.485252 & 1.441356 & -0.994885 \\ \text { H } & 0.528582 & 2.358561 & 0.204285 \\ \text { H } & -0.251203 & 1.358942 & -1.092741 \\ \text { H } & 1.870714 & 0.603033 & 1.252831 \\ \text { H } & -0.477306 & 0.741793 & 1.499112 \\ \text { H } & -1.222251 & -1.592711 & 0.698862 \\ \text { H } & -0.743817 & -1.150297 & -0.926063 \\ \text { H } & -3.202415 & -0.816405 & -0.565609 \\ \text { H } & -2.88009 & 0.305816 & 0.754125 \\ \text { H } & -2.446553 & 0.733571 & -0.90652 \\ \text { H } & 2.182819 & -1.804477 & 0.285124 \\ \text { H } & 1.437221 & -1.229578 & -1.209789 \\ & 2.970836 & -0.53235 & -0.647414\end{array}$




$\begin{array}{llll}\text { 6CA_5CB_td_PBE } & & \\ \text { C } & 1.13757 & 1.41012 & \\ \text { C } & 0.910742 & -0.294831 & -0.417381 \\ \text { C } & -0.154552 & 0.181336 & 0.373236 \\ \mathrm{C} & -1.520287 & 0.521771 & -0.15406 \\ \mathrm{C} & -2.441713 & -0.703591 & 0.042183 \\ \mathrm{C} & 2.056943 & -1.122688 & 0.083087 \\ \mathrm{H} & 1.648778 & 1.60671 & -0.899841 \\ \mathrm{H} & 1.822897 & 1.436863 & 0.908802 \\ \mathrm{H} & 0.364936 & 2.193394 & 0.114026 \\ \mathrm{H} & 0.740685 & -0.301459 & -1.503189 \\ \mathrm{H} & -0.064355 & 0.042767 & 1.460765 \\ \mathrm{H} & -1.465816 & 0.792435 & -1.222317 \\ \mathrm{H} & -1.932078 & 1.383724 & 0.397278 \\ \mathrm{H} & -3.450349 & -0.450091 & -0.315977 \\ \mathrm{H} & -2.087271 & -1.575806 & -0.528186 \\ \mathrm{H} & -2.525096 & -0.987154 & 1.102927 \\ \mathrm{H} & 3.000621 & -0.865475 & -0.421046 \\ \mathrm{H} & 2.184182 & -1.055224 & 1.173671 \\ \mathrm{H} & 1.830644 & -2.17338 & -0.171997 \\ & & & \\ \text { 6CA_5CB_td_CC } & & \\ & & & \\ \mathrm{C} & 1.124356 & 1.412406 & 0.04165 \\ \mathrm{C} & 0.891342 & -0.310616 & -0.413906 \\ \mathrm{C} & -0.146252 & 0.164453 & 0.382161 \\ \mathrm{C} & -1.511649 & 0.517635 & -0.14104 \\ \mathrm{C} & -2.438497 & -0.696227 & 0.034223 \\ \mathrm{C} & 2.063063 & -1.107103 & 0.083622 \\ \mathrm{H} & 1.613338 & 1.586481 & -0.915393 \\ \mathrm{H} & 1.819876 & 1.42995 & 0.870573 \\ \mathrm{H} & 0.354055 & 2.175321 & 0.128722 \\ \mathrm{H} & 0.714757 & -0.321425 & -1.485233 \\ \mathrm{H} & -0.045509 & 0.039782 & 1.457272 \\ \mathrm{H} & -1.445337 & 0.797635 & -1.193957 \\ \mathrm{H} & -1.906983 & 1.370059 & 0.412854 \\ \mathrm{H} & -3.434262 & -0.440531 & -0.321894 \\ \mathrm{H} & -2.081363 & -1.552061 & -0.538153 \\ \mathrm{H} & -2.518955 & -0.985234 & 1.081945 \\ & 2.982437 & -0.814025 & -0.419954 \\ & 2.186243 & -1.016028 & 1.161109 \\ 1.867534 & -2.15321 & -0.158149\end{array}$




$\begin{array}{llll}\text { 6CA_5CB_tg_PBE } & & \\ & & & \\ \mathrm{C} & 0.600743 & 1.402546 & -0.265682 \\ \mathrm{C} & 0.860807 & -0.371251 & -0.276059 \\ \mathrm{C} & -0.036234 & 0.037585 & 0.731294 \\ \mathrm{C} & -1.532375 & -0.128172 & 0.704006 \\ \mathrm{C} & -2.189201 & -0.377603 & -0.654542 \\ \mathrm{C} & 2.309847 & -0.683093 & -0.049629 \\ \mathrm{H} & 0.974421 & 1.392188 & -1.30477 \\ \mathrm{H} & 1.316814 & 1.91513 & 0.383384 \\ \mathrm{H} & -0.382079 & 1.890306 & -0.332042 \\ \mathrm{H} & 0.416615 & -0.738477 & -1.210849 \\ \mathrm{H} & 0.403407 & 0.251863 & 1.715687 \\ \mathrm{H} & -1.992364 & 0.72917 & 1.226181 \\ \mathrm{H} & -1.706992 & -0.991252 & 1.379082 \\ \mathrm{H} & -3.276014 & -0.479457 & -0.522949 \\ \mathrm{H} & -2.030305 & 0.457959 & -1.357455 \\ \mathrm{H} & -1.832651 & -1.30587 & -1.128508 \\ \mathrm{H} & 2.935221 & -0.388848 & -0.905658 \\ \mathrm{H} & 2.702254 & -0.232082 & 0.874111 \\ \mathrm{H} & 2.390157 & -1.780699 & 0.04746 \\ & & & \\ \text { 6CA_5CB_tg_CC } & & -0.017 \\ \mathrm{H} & & & \\ \mathrm{C} & 0.598197 & 1.398926 & -0.246983 \\ \mathrm{C} & 0.844452 & -0.379915 & -0.273263 \\ \mathrm{C} & -0.033436 & 0.005595 & 0.735227 \\ \mathrm{C} & -1.532199 & -0.14684 & 0.704866 \\ \mathrm{C} & -2.16862 & -0.350715 & -0.668029 \\ \mathrm{C} & 2.304343 & -0.666392 & -0.063806 \\ \mathrm{H} & 0.837215 & 1.408084 & -1.309503 \\ \mathrm{H} & 1.386016 & 1.851588 & 0.340995 \\ \mathrm{H} & -0.35714 & 1.907173 & -0.147611 \\ \mathrm{H} & 0.40864 & -0.731856 & -1.201461 \\ \mathrm{H} & 0.403773 & 0.21757 & 1.7071 \\ \mathrm{H} & -1.977173 & 0.703869 & 1.224665 \\ \mathrm{H} & -1.723349 & -1.016025 & 1.34342 \\ \mathrm{H} & -3.24765 & -0.428889 & -0.553951 \\ \mathrm{H} & -1.973366 & 0.487066 & -1.339659 \\ \mathrm{H} & -1.823875 & -1.268517 & -1.143756 \\ & 2.901943 & -0.303542 & -0.897974 \\ & 2.671948 & -0.239555 & 0.867481 \\ & 2.416587 & -1.750921 & -0.017814\end{array}$




\section{Branching}

$\begin{array}{llll}\text { 9C_6CA_gd_PBE } & & \\ \text { C } & -2.268393 & 0.338368 & -0.268351 \\ \text { C } & -1.251066 & -0.430953 & 0.58434 \\ \text { C } & -0.050299 & -0.875142 & -0.276661 \\ \text { C } & 1.679348 & -0.9315 & 0.178101 \\ \text { C } & 1.144373 & 0.221082 & -0.493136 \\ \text { H } & -1.850431 & 1.264081 & -0.69276 \\ \text { H } & -2.648215 & -0.280178 & -1.097202 \\ \text { H } & -3.130466 & 0.614572 & 0.357621 \\ \text { H } & -0.902733 & 0.174573 & 1.435212 \\ \text { H } & -1.725617 & -1.330373 & 1.009183 \\ \text { H } & 2.203664 & -1.723753 & -0.368676 \\ \text { H } & 1.788518 & -0.923151 & 1.269791 \\ \text { H } & -0.268227 & -1.266037 & -1.281441 \\ \text { H } & 0.319692 & -1.841329 & 0.222858 \\ \text { H } & 1.36735 & 0.281422 & -1.564168 \\ \text { C } & 1.036936 & 1.559839 & 0.207536 \\ \text { H } & 1.974173 & 2.108902 & 0.023536 \\ \text { H } & 0.215089 & 2.164359 & -0.200896 \\ \text { H } & 0.911801 & 1.466751 & 1.295973\end{array}$

9C_6CA_gd_CC

$\begin{array}{llll}\text { C } & -2.25528 & 0.330316 & -0.271252 \\ \mathrm{C} & -1.245529 & -0.43757 & 0.583626 \\ \mathrm{C} & -0.041862 & -0.88748 & -0.262989 \\ \mathrm{C} & 1.699826 & -0.899843 & 0.168955 \\ \mathrm{C} & 1.121963 & 0.220083 & -0.496019 \\ \mathrm{H} & -1.825038 & 1.231252 & -0.705876 \\ \mathrm{H} & -2.632339 & -0.295106 & -1.08051 \\ \mathrm{H} & -3.102453 & 0.624715 & 0.345817 \\ \mathrm{H} & -0.906514 & 0.160911 & 1.427646 \\ \mathrm{H} & -1.719664 & -1.328254 & 0.996459 \\ \mathrm{H} & 2.206054 & -1.686833 & -0.376392 \\ \mathrm{H} & 1.819642 & -0.886164 & 1.246416 \\ \mathrm{H} & -0.260647 & -1.293735 & -1.247152 \\ \mathrm{H} & 0.350971 & -1.810677 & 0.261631 \\ \mathrm{H} & 1.327432 & 0.281737 & -1.557864 \\ \mathrm{C} & 1.009952 & 1.557956 & 0.206922 \\ \mathrm{H} & 1.926656 & 2.112513 & 0.009126 \\ \mathrm{H} & 0.176363 & 2.138356 & -0.179153 \\ \mathrm{H} & 0.905121 & 1.450515 & 1.284393\end{array}$




$\begin{array}{llll}\text { 9C_6CA_gt_PBE } & & \\ \text { C } & -2.620093 & 0.097969 & 0.06033 \\ \text { C } & -1.162008 & 0.565226 & 0.105138 \\ \text { C } & -0.196687 & -0.490793 & -0.449052 \\ \text { C } & 1.104626 & -1.290758 & 0.45904 \\ \text { C } & 1.369309 & -0.163533 & -0.413755 \\ \text { H } & -2.930301 & -0.172482 & -0.961546 \\ \text { H } & -2.793121 & -0.771703 & 0.716117 \\ \text { H } & -3.280888 & 0.907677 & 0.403146 \\ \text { H } & -1.018271 & 1.469678 & -0.51085 \\ \text { H } & -0.869793 & 0.836855 & 1.132716 \\ \text { H } & 1.289611 & -2.319806 & 0.128702 \\ \text { H } & 0.990605 & -1.12395 & 1.537695 \\ \text { H } & -0.425076 & -0.934802 & -1.429396 \\ \text { H } & -0.41746 & -1.435308 & 0.210052 \\ \text { C } & 1.806864 & 1.182139 & 0.132242 \\ \text { H } & 2.906354 & 1.193054 & 0.187516 \\ \text { H } & 1.501067 & 1.996345 & -0.54136 \\ \text { H } & 1.413165 & 1.38439 & 1.138655 \\ \text { H } & 1.822042 & -0.431445 & -1.375109 \\ & & & \\ \text { 9C_6CA_gt_CC } & & \\ & & & \\ \text { C } & -2.609343 & -0.092171 & -0.050532 \\ \text { C } & -1.155316 & -0.55711 & -0.121203 \\ \text { C } & -0.199527 & 0.501631 & 0.437041 \\ \text { C } & 1.130363 & 1.277208 & -0.455768 \\ \text { C } & 1.354957 & 0.159696 & 0.418581 \\ \text { H } & -2.898899 & 0.14425 & 0.973385 \\ \text { H } & -2.775956 & 0.790836 & -0.669752 \\ \text { H } & -3.266184 & -0.881548 & -0.410852 \\ \text { H } & -1.01125 & -1.465355 & 0.465018 \\ \text { H } & -0.880005 & -0.791882 & -1.150038 \\ \text { H } & 1.304264 & 2.293895 & -0.124706 \\ \text { H } & 1.017301 & 1.113295 & -1.521709 \\ \text { H } & -0.433115 & 0.931652 & 1.407823 \\ \text { H } & -0.397744 & 1.422546 & -0.22344 \\ \text { C } & 1.782999 & -1.189583 & -0.123054 \\ \text { H } & 2.870492 & -1.208357 & -0.177004 \\ \text { H } & 1.46442 & -1.987237 & 0.545034 \\ \text { H } & 1.38638 & -1.379387 & -1.118053 \\ \text { H } & 1.795502 & 0.419259 & 1.37391 \\ & & & \end{array}$




\begin{tabular}{|c|c|c|c|}
\hline \multicolumn{4}{|c|}{ 9C_6CA_gu_PBE } \\
\hline $\mathrm{C}$ & -2.027702 & 0.324258 & 0.620073 \\
\hline $\mathrm{C}$ & -1.301198 & 0.143961 & -0.71718 \\
\hline C & -0.040376 & -0.739409 & -0.692476 \\
\hline C & 0.919011 & -1.11013 & 0.753114 \\
\hline $\mathrm{C}$ & 1.339103 & -0.115638 & -0.228356 \\
\hline $\mathrm{H}$ & -2.356 & -0.64141 & 1.041147 \\
\hline $\mathrm{H}$ & -1.416992 & 0.846133 & 1.374752 \\
\hline $\mathrm{H}$ & -2.930389 & 0.933447 & 0.466166 \\
\hline $\mathrm{H}$ & -1.981738 & -0.334334 & -1.440526 \\
\hline $\mathrm{H}$ & -1.014056 & 1.119628 & -1.142723 \\
\hline $\mathrm{H}$ & 1.354236 & -2.116785 & 0.753565 \\
\hline $\mathrm{H}$ & 0.418731 & -0.779202 & 1.671361 \\
\hline $\mathrm{H}$ & 0.1518 & -1.401044 & -1.549033 \\
\hline $\mathrm{H}$ & -0.32494 & -1.588531 & 0.092053 \\
\hline $\mathrm{C}$ & 1.390424 & 1.361714 & 0.107177 \\
\hline $\mathrm{H}$ & 2.371671 & 1.589292 & 0.551136 \\
\hline $\mathrm{H}$ & 1.292157 & 1.968396 & -0.80564 \\
\hline $\mathrm{H}$ & 0.613545 & 1.66816 & 0.822347 \\
\hline $\mathrm{H}$ & 2.146406 & -0.452284 & -0.888714 \\
\hline \multicolumn{4}{|c|}{ 9C_6CA_gu_CC } \\
\hline $\mathrm{C}$ & -2.015533 & 0.309256 & 0.625443 \\
\hline $\mathrm{C}$ & -1.296474 & 0.147437 & -0.714327 \\
\hline $\mathrm{C}$ & -0.045167 & -0.746632 & -0.685652 \\
\hline $\mathrm{C}$ & 0.94289 & -1.09725 & 0.748121 \\
\hline $\mathrm{C}$ & 1.330189 & -0.11116 & -0.230755 \\
\hline $\mathrm{H}$ & -2.332909 & -0.657018 & 1.022496 \\
\hline $\mathrm{H}$ & -1.402303 & 0.809189 & 1.375361 \\
\hline $\mathrm{H}$ & -2.909035 & 0.914071 & 0.484388 \\
\hline $\mathrm{H}$ & -1.974093 & -0.320148 & -1.429131 \\
\hline $\mathrm{H}$ & -1.014746 & 1.11582 & -1.12641 \\
\hline $\mathrm{H}$ & 1.364061 & -2.095308 & 0.730582 \\
\hline $\mathrm{H}$ & 0.440558 & -0.785727 & 1.656357 \\
\hline $\mathrm{H}$ & 0.151749 & -1.376269 & -1.548907 \\
\hline $\mathrm{H}$ & -0.325165 & -1.576723 & 0.075213 \\
\hline $\mathrm{C}$ & 1.36667 & 1.364984 & 0.10617 \\
\hline $\mathrm{H}$ & 2.335594 & 1.596191 & 0.546142 \\
\hline $\mathrm{H}$ & 1.256693 & 1.96089 & -0.798023 \\
\hline $\mathrm{H}$ & 0.59135 & 1.64809 & 0.813923 \\
\hline $\mathrm{H}$ & 2.122797 & -0.432873 & -0.895993 \\
\hline
\end{tabular}




\begin{tabular}{|c|c|c|c|}
\hline \multicolumn{4}{|c|}{ 9C_6CA_td_PBE } \\
\hline C & 2.211022 & 0.806164 & -0.090152 \\
\hline $\mathrm{C}$ & 1.562259 & -0.581845 & -0.022778 \\
\hline $\mathrm{C}$ & 0.135359 & -0.493061 & 0.540044 \\
\hline $\mathrm{C}$ & -1.288423 & -1.260997 & -0.200564 \\
\hline $\mathrm{C}$ & -0.958898 & 0.130675 & -0.433561 \\
\hline $\mathrm{C}$ & -1.826287 & 1.247133 & 0.114999 \\
\hline $\mathrm{H}$ & 1.669784 & 1.485318 & -0.768022 \\
\hline $\mathrm{H}$ & 2.266043 & 1.276919 & 0.904142 \\
\hline $\mathrm{H}$ & 3.24024 & 0.708662 & -0.466782 \\
\hline $\mathrm{H}$ & 1.525975 & -1.047788 & -1.022982 \\
\hline $\mathrm{H}$ & 2.155426 & -1.251414 & 0.621238 \\
\hline $\mathrm{H}$ & -2.06579 & -1.526966 & 0.527511 \\
\hline $\mathrm{H}$ & -0.495542 & 0.330648 & -1.407835 \\
\hline $\mathrm{H}$ & -0.980974 & -2.037673 & -0.910562 \\
\hline $\mathrm{H}$ & -0.019497 & -0.042691 & 1.532809 \\
\hline $\mathrm{H}$ & -0.150458 & -1.59331 & 0.813515 \\
\hline $\mathrm{H}$ & -2.272798 & 0.983879 & 1.086786 \\
\hline $\mathrm{H}$ & -1.239485 & 2.169719 & 0.234971 \\
\hline $\mathrm{H}$ & -2.643117 & 1.456285 & -0.592718 \\
\hline \multicolumn{4}{|c|}{ 9C_6CA_td_CC } \\
\hline $\mathrm{C}$ & 2.184624 & 0.79933 & -0.102329 \\
\hline $\mathrm{C}$ & 1.550917 & -0.588782 & -0.008785 \\
\hline $\mathrm{C}$ & 0.126264 & -0.506043 & 0.549528 \\
\hline $\mathrm{C}$ & -1.302112 & -1.243065 & -0.216598 \\
\hline $\mathrm{C}$ & -0.937728 & 0.130014 & -0.432604 \\
\hline $\mathrm{C}$ & -1.787212 & 1.256114 & 0.120619 \\
\hline $\mathrm{H}$ & 1.636649 & 1.448042 & -0.786201 \\
\hline $\mathrm{H}$ & 2.222686 & 1.279216 & 0.875662 \\
\hline $\mathrm{H}$ & 3.204578 & 0.709829 & -0.471431 \\
\hline $\mathrm{H}$ & 1.517184 & -1.066224 & -0.990735 \\
\hline $\mathrm{H}$ & 2.142795 & -1.232232 & 0.642568 \\
\hline $\mathrm{H}$ & -2.070214 & -1.498504 & 0.505071 \\
\hline $\mathrm{H}$ & -0.471596 & 0.326352 & -1.391635 \\
\hline $\mathrm{H}$ & -0.999149 & -2.014442 & -0.914375 \\
\hline $\mathrm{H}$ & -0.023345 & -0.064278 & 1.532262 \\
\hline $\mathrm{H}$ & -0.166422 & -1.593281 & 0.785705 \\
\hline $\mathrm{H}$ & -2.226855 & 0.985042 & 1.0804 \\
\hline $\mathrm{H}$ & -1.183681 & 2.152354 & 0.251583 \\
\hline $\mathrm{H}$ & -2.591147 & 1.48272 & -0.577853 \\
\hline
\end{tabular}




\begin{tabular}{|c|c|c|c|}
\hline \multicolumn{4}{|c|}{ 9C_6CA_tg_PBE } \\
\hline C & -2.276899 & 0.162825 & -0.563717 \\
\hline C & -1.456543 & -0.754866 & 0.34617 \\
\hline C & -0.062255 & -0.233969 & 0.737746 \\
\hline C & 0.634766 & 1.317915 & 0.215687 \\
\hline C & 0.959704 & 0.055821 & -0.429434 \\
\hline C & 2.34439 & -0.553484 & -0.313244 \\
\hline $\mathrm{H}$ & -2.521176 & 1.120489 & -0.074004 \\
\hline $\mathrm{H}$ & -1.778813 & 0.370673 & -1.5255 \\
\hline $\mathrm{H}$ & -3.233117 & -0.324665 & -0.803931 \\
\hline $\mathrm{H}$ & -2.006968 & -0.953435 & 1.280065 \\
\hline $\mathrm{H}$ & -1.283454 & -1.730646 & -0.139918 \\
\hline $\mathrm{H}$ & 1.326023 & 1.752592 & 0.949338 \\
\hline $\mathrm{H}$ & 0.463825 & -0.091498 & -1.397205 \\
\hline $\mathrm{H}$ & -0.129287 & 1.977874 & -0.210184 \\
\hline $\mathrm{H}$ & 0.483931 & -0.740445 & 1.547627 \\
\hline $\mathrm{H}$ & -0.282918 & 0.780062 & 1.303305 \\
\hline $\mathrm{H}$ & 2.799225 & -0.362315 & 0.671583 \\
\hline $\mathrm{H}$ & 2.302377 & -1.640704 & -0.475882 \\
\hline $\mathrm{H}$ & 3.001379 & -0.123433 & -1.084544 \\
\hline \multicolumn{4}{|c|}{ 9C_6CA_tg_CC } \\
\hline C & -2.263373 & 0.180108 & -0.544259 \\
\hline C & -1.441989 & -0.773646 & 0.3214 \\
\hline C & -0.062419 & -0.240166 & 0.732492 \\
\hline C & 0.63728 & 1.313752 & 0.219248 \\
\hline$C$ & 0.94668 & 0.063218 & -0.429775 \\
\hline $\mathrm{C}$ & 2.327731 & -0.549621 & -0.310513 \\
\hline $\mathrm{H}$ & -2.489518 & 1.107312 & -0.014482 \\
\hline $\mathrm{H}$ & -1.767283 & 0.422499 & -1.485308 \\
\hline $\mathrm{H}$ & -3.212675 & -0.288922 & -0.794426 \\
\hline $\mathrm{H}$ & -1.986595 & -1.006574 & 1.23653 \\
\hline $\mathrm{H}$ & -1.266387 & -1.715109 & -0.201347 \\
\hline $\mathrm{H}$ & 1.314432 & 1.724597 & 0.960008 \\
\hline $\mathrm{H}$ & 0.461098 & -0.073945 & -1.389417 \\
\hline $\mathrm{H}$ & -0.11776 & 1.974875 & -0.187258 \\
\hline $\mathrm{H}$ & 0.474198 & -0.753788 & 1.526493 \\
\hline $\mathrm{H}$ & -0.293545 & 0.755944 & 1.282766 \\
\hline $\mathrm{H}$ & 2.759844 & -0.362574 & 0.672528 \\
\hline $\mathrm{H}$ & 2.276179 & -1.624905 & -0.471866 \\
\hline $\mathrm{H}$ & 2.984546 & -0.121274 & -1.065782 \\
\hline
\end{tabular}




$\begin{array}{llll}\text { 9C_6CA_tt_PBE } & & \\ \text { C } & 2.71907 & -0.152397 & -0.261328 \\ \mathrm{C} & 1.403539 & -0.360051 & 0.493082 \\ \mathrm{C} & 0.166135 & -0.002175 & -0.339463 \\ \mathrm{C} & -0.924866 & 1.351135 & 0.030639 \\ \mathrm{C} & -1.225149 & -0.010194 & 0.428241 \\ \mathrm{C} & -2.367207 & -0.782472 & -0.203553 \\ \mathrm{H} & 2.749497 & -0.737636 & -1.194075 \\ \mathrm{H} & 2.886246 & 0.908578 & -0.51226 \\ \mathrm{H} & 3.563554 & -0.480834 & 0.3622 \\ \mathrm{H} & 1.277361 & -1.417944 & 0.783101 \\ \mathrm{H} & 1.386382 & 0.2228 & 1.430983 \\ \mathrm{H} & -1.405341 & 1.782023 & -0.857341 \\ \mathrm{H} & -1.043586 & -0.223788 & 1.489075 \\ \mathrm{H} & -0.449095 & 2.047835 & 0.730841 \\ \mathrm{H} & 0.013476 & -0.496374 & -1.311503 \\ \mathrm{H} & 0.391842 & 1.07438 & -0.747654 \\ \mathrm{H} & -2.513327 & -0.513488 & -1.261515 \\ \mathrm{H} & -2.18627 & -1.865407 & -0.136129 \\ \mathrm{H} & -3.299861 & -0.563218 & 0.338572 \\ & & & \\ 9 \mathrm{C} \_6 \mathrm{CA} \mathrm{tt}+\mathrm{CC} & & \\ & & & \\ \mathrm{C} & 2.702448 & -0.15802 & -0.262984 \\ \mathrm{C} & 1.393511 & -0.343856 & 0.501029 \\ \mathrm{C} & 0.165383 & 0.004359 & -0.342847 \\ \mathrm{C} & -0.941916 & 1.346894 & 0.021868 \\ \mathrm{C} & -1.209877 & -0.006395 & 0.429831 \\ \mathrm{C} & -2.339175 & -0.797214 & -0.19903 \\ \mathrm{H} & 2.720062 & -0.765765 & -1.167659 \\ \mathrm{H} & 2.854039 & 0.88558 & -0.544254 \\ \mathrm{H} & 3.541557 & -0.460609 & 0.360458 \\ \mathrm{H} & 1.27096 & -1.381118 & 0.81752 \\ \mathrm{H} & 1.381502 & 0.267481 & 1.406484 \\ \mathrm{H} & -1.412513 & 1.752549 & -0.866931 \\ \mathrm{H} & -1.031055 & -0.204587 & 1.480964 \\ \mathrm{H} & -0.473225 & 2.048717 & 0.701361 \\ \mathrm{H} & 0.024325 & -0.500096 & -1.296228 \\ \mathrm{H} & 0.376183 & 1.07024 & -0.732901 \\ \mathrm{H} & -2.464747 & -0.539672 & -1.250599 \\ & -2.139905 & -1.864345 & -0.119987 \\ & -3.269434 & -0.582985 & 0.32457\end{array}$




\begin{tabular}{|c|c|c|c|}
\hline \multicolumn{4}{|c|}{ 8CA_5CB_gd_PBE } \\
\hline C & 1.185937 & 0.275305 & -0.516414 \\
\hline C & 1.222454 & -1.326861 & 0.245934 \\
\hline C & 0.002982 & -0.79852 & -0.326209 \\
\hline C & -1.203622 & -0.423769 & 0.526787 \\
\hline C & -2.256957 & 0.382053 & -0.243536 \\
\hline $\mathrm{H}$ & 2.203205 & -0.195329 & -0.185096 \\
\hline $\mathrm{H}$ & 1.410195 & -1.219182 & 1.321926 \\
\hline $\mathrm{H}$ & -1.645286 & -1.379104 & 0.862004 \\
\hline $\mathrm{H}$ & -0.260908 & -1.227589 & -1.30065 \\
\hline $\mathrm{H}$ & 1.830384 & -2.063503 & -0.293629 \\
\hline $\mathrm{H}$ & -0.88546 & 0.101309 & 1.44304 \\
\hline $\mathrm{H}$ & -1.877012 & 1.358115 & -0.584668 \\
\hline $\mathrm{H}$ & -2.613157 & -0.17015 & -1.128003 \\
\hline $\mathrm{H}$ & -3.128301 & 0.572949 & 0.3996 \\
\hline C & 1.102999 & 1.583158 & 0.264152 \\
\hline $\mathrm{H}$ & 0.216739 & 2.145863 & -0.062086 \\
\hline $\mathrm{H}$ & 1.028364 & 1.419543 & 1.348753 \\
\hline $\mathrm{H}$ & 1.997716 & 2.191403 & 0.068413 \\
\hline $\mathrm{H}$ & 1.400765 & 0.317486 & -1.593887 \\
\hline \multicolumn{4}{|c|}{ 8CA_5CB_gd_CC } \\
\hline C & 1.185765 & 0.274937 & -0.518089 \\
\hline C & 1.208783 & -1.333027 & 0.244055 \\
\hline C & 0.008221 & -0.792135 & -0.323378 \\
\hline C & -1.18904 & -0.41102 & 0.535033 \\
\hline C & -2.245622 & 0.36832 & -0.249714 \\
\hline $\mathrm{H}$ & 2.180534 & -0.180953 & -0.174596 \\
\hline $\mathrm{H}$ & 1.403266 & -1.222936 & 1.305256 \\
\hline $\mathrm{H}$ & -1.621675 & -1.347125 & 0.894577 \\
\hline $\mathrm{H}$ & -0.25825 & -1.214499 & -1.286246 \\
\hline $\mathrm{H}$ & 1.816821 & -2.046127 & -0.299834 \\
\hline $\mathrm{H}$ & -0.865324 & 0.14118 & 1.417807 \\
\hline $\mathrm{H}$ & -1.86197 & 1.316811 & -0.62496 \\
\hline $\mathrm{H}$ & -2.602883 & -0.213474 & -1.099743 \\
\hline $\mathrm{H}$ & -3.099123 & 0.582965 & 0.390426 \\
\hline C & 1.085599 & 1.581736 & 0.263939 \\
\hline $\mathrm{H}$ & 0.179594 & 2.111781 & -0.016102 \\
\hline $\mathrm{H}$ & 1.072561 & 1.406331 & 1.337618 \\
\hline $\mathrm{H}$ & 1.945664 & 2.205005 & 0.028722 \\
\hline $\mathrm{H}$ & 1.388545 & 0.328172 & -1.583999 \\
\hline
\end{tabular}




$\begin{array}{llll}\text { 8CA_5CB_gt_PBE } & & \\ & & & \\ \text { C } & 1.41942 & 0.231441 & 0.400594 \\ \text { C } & 0.411205 & 1.419551 & -0.448693 \\ \text { C } & -0.159935 & 0.445254 & 0.465005 \\ \text { C } & -1.070337 & -0.671018 & -0.029963 \\ \text { H } & 1.888999 & 1.070736 & -0.278896 \\ \text { H } & 0.397316 & 1.219874 & -1.528003 \\ \text { H } & -0.962504 & -1.536591 & 0.64573 \\ \text { H } & -0.445418 & 0.854912 & 1.441441 \\ \text { H } & 0.594236 & 2.454525 & -0.134678 \\ \text { C } & -2.534662 & -0.207101 & -0.057567 \\ \text { H } & -3.182022 & -1.033848 & -0.384409 \\ \text { H } & -2.878549 & 0.110858 & 0.939642 \\ \text { H } & -2.682671 & 0.632754 & -0.755544 \\ \text { H } & -0.757638 & -1.00508 & -1.033555 \\ \text { C } & 1.933451 & -1.071716 & -0.203152 \\ \text { H } & 3.032472 & -1.07137 & -0.221311 \\ \text { H } & 1.590018 & -1.909849 & 0.421758 \\ \text { H } & 1.567054 & -1.23346 & -1.226535 \\ \text { H } & 1.84386 & 0.568067 & 1.357016 \\ & & & \\ \text { 8CA_5CB_gt_CC } & & \\ & & & \\ \text { C } & 1.418586 & 0.242272 & 0.392168 \\ \text { C } & 0.382873 & 1.419548 & -0.445189 \\ \text { C } & -0.153895 & 0.444385 & 0.467031 \\ \text { C } & -1.056067 & -0.675061 & -0.025528 \\ \text { H } & 1.867643 & 1.055728 & -0.292347 \\ \text { H } & 0.369902 & 1.221719 & -1.511542 \\ \text { H } & -0.948517 & -1.529398 & 0.644483 \\ \text { H } & -0.429831 & 0.843278 & 1.436967 \\ \text { H } & 0.577118 & 2.438315 & -0.131383 \\ \text { C } & -2.51561 & -0.212918 & -0.057187 \\ \text { H } & -3.155205 & -1.028473 & -0.388801 \\ \text { H } & -2.852226 & 0.097772 & 0.931981 \\ \text { H } & -2.650912 & 0.622975 & -0.744862 \\ \text { H } & -0.744007 & -1.000166 & -1.019017 \\ \text { C } & 1.920781 & -1.069659 & -0.204104 \\ \text { H } & 3.008264 & -1.080502 & -0.189187 \\ \text { H } & 1.549932 & -1.899763 & 0.392935 \\ \text { H } & 1.586401 & -1.199814 & -1.230848 \\ \text { H } & 1.841422 & 0.56693 & 1.338475\end{array}$




\begin{tabular}{llll} 
8CA_5CB_td_PBE & & \\
& & & \\
$\mathrm{C}$ & -1.926376 & -1.195954 & -0.142002 \\
$\mathrm{C}$ & -0.992024 & -0.175645 & 0.498033 \\
$\mathrm{C}$ & -0.864359 & 1.503983 & -0.052057 \\
$\mathrm{C}$ & 0.09073 & 0.486348 & -0.45286 \\
$\mathrm{C}$ & 1.532631 & 0.512496 & 0.038642 \\
$\mathrm{C}$ & 2.195028 & -0.869333 & 0.023907 \\
$\mathrm{H}$ & -2.735435 & -1.48309 & 0.544344 \\
$\mathrm{H}$ & -1.338227 & -2.092108 & -0.391569 \\
$\mathrm{H}$ & -2.371086 & -0.810563 & -1.072136 \\
$\mathrm{H}$ & -0.491924 & -0.422923 & 1.446833 \\
$\mathrm{H}$ & -1.697414 & 0.667135 & 0.921738 \\
$\mathrm{H}$ & -0.601349 & 2.214462 & 0.743021 \\
$\mathrm{H}$ & 2.084071 & 1.203744 & -0.622398 \\
$\mathrm{H}$ & -0.048929 & 0.1051 & -1.472816 \\
$\mathrm{H}$ & -1.712069 & 1.765696 & -0.696297 \\
$\mathrm{H}$ & 1.569511 & 0.955646 & 1.050618 \\
$\mathrm{H}$ & 1.69711 & -1.574753 & 0.71023 \\
$\mathrm{H}$ & 2.187417 & -1.310336 & -0.985771 \\
$\mathrm{H}$ & 3.244543 & -0.789384 & 0.342224 \\
& & & \\
8CA_5CB_td_CC & & \\
& & & \\
$\mathrm{C}$ & -1.88553 & -1.212083 & -0.142742 \\
$\mathrm{C}$ & -0.984195 & -0.16768 & 0.506102 \\
$\mathrm{C}$ & -0.857372 & 1.509899 & -0.061282 \\
$\mathrm{C}$ & 0.081753 & 0.489814 & -0.449966 \\
$\mathrm{C}$ & 1.518044 & 0.516286 & 0.046398 \\
$\mathrm{C}$ & 2.165018 & -0.868311 & 0.018482 \\
$\mathrm{H}$ & -2.665816 & -1.529739 & 0.544848 \\
$\mathrm{H}$ & -1.277956 & -2.072879 & -0.415329 \\
$\mathrm{H}$ & -2.349439 & -0.816068 & -1.045349 \\
$\mathrm{H}$ & -0.494925 & -0.405801 & 1.447027 \\
$\mathrm{H}$ & -1.702522 & 0.655194 & 0.885959 \\
$\mathrm{H}$ & -0.607698 & 2.203969 & 0.733875 \\
$\mathrm{H}$ & 2.073371 & 1.203868 & -0.594278 \\
$\mathrm{H}$ & -0.052496 & 0.110891 & -1.45811 \\
$\mathrm{H}$ & -1.699323 & 1.762361 & -0.695008 \\
$\mathrm{H}$ & 1.542933 & 0.933867 & 1.056612 \\
& 1.651288 & -1.564841 & 0.683261 \\
\hline & 3.154887 & -1.284591 & -0.989179 \\
& & -0.803786 & 0.343709
\end{tabular}




$\begin{array}{llll}\text { 8CA_5CB_tg_PBE } & & \\ \text { C } & 2.375552 & -0.611819 & -0.125175 \\ \text { C } & 0.977373 & -0.126492 & -0.485865 \\ \text { C } & 0.292655 & 1.39248 & 0.106312 \\ \text { C } & -0.02235 & 0.107517 & 0.714182 \\ \text { C } & -1.416199 & -0.51527 & 0.633465 \\ \text { C } & -2.239496 & -0.195364 & -0.616461 \\ \text { H } & 3.042507 & -0.597906 & -0.998707 \\ \text { H } & 2.296395 & -1.646469 & 0.242402 \\ \text { H } & 2.823517 & -0.000574 & 0.673218 \\ \text { H } & 0.421983 & -0.619887 & -1.29825 \\ \text { H } & 1.142057 & 0.903649 & -1.043378 \\ \text { H } & -0.421803 & 1.858567 & -0.584179 \\ \text { H } & -1.295579 & -1.60623 & 0.747313 \\ \text { H } & 0.5031 & -0.07989 & 1.659004 \\ \text { H } & 1.071471 & 2.034996 & 0.533028 \\ \text { H } & -1.957117 & -0.17835 & 1.534794 \\ \text { H } & -2.482959 & 0.876692 & -0.702085 \\ \text { H } & -1.750399 & -0.5187 & -1.552411 \\ \text { H } & -3.198381 & -0.732205 & -0.569508\end{array}$

8CA_5CB_tg_CC

$\begin{array}{llll}\text { C } & 2.365681 & -0.601191 & -0.133549 \\ \mathrm{C} & 0.973507 & -0.10223 & -0.50059 \\ \mathrm{C} & 0.268653 & 1.387117 & 0.147267 \\ \mathrm{C} & -0.014841 & 0.085712 & 0.704561 \\ \mathrm{C} & -1.39374 & -0.5592 & 0.608058 \\ \mathrm{C} & -2.235997 & -0.166938 & -0.604896 \\ \mathrm{H} & 3.023716 & -0.58578 & -0.99914 \\ \mathrm{H} & 2.281334 & -1.623371 & 0.230916 \\ \mathrm{H} & 2.802133 & 0.011718 & 0.654223 \\ \mathrm{H} & 0.441518 & -0.57901 & -1.319045 \\ \mathrm{H} & 1.146 & 0.933148 & -0.99636 \\ \mathrm{H} & -0.431451 & 1.856956 & -0.533197 \\ \mathrm{H} & -1.249683 & -1.64095 & 0.636368 \\ \mathrm{H} & 0.508934 & -0.120606 & 1.631986 \\ \mathrm{H} & 1.035741 & 2.016342 & 0.582651 \\ \mathrm{H} & -1.925346 & -0.296208 & 1.524356 \\ \mathrm{H} & -2.494573 & 0.892665 & -0.59838 \\ \mathrm{H} & -1.746864 & -0.401283 & -1.552626 \\ \mathrm{H} & -3.171035 & -0.723238 & -0.586863\end{array}$




$\begin{array}{llll}\text { 8CA_5CB_tt_PBE } & & \\ & & & \\ \mathrm{C} & -2.447578 & -0.630079 & -0.296034 \\ \mathrm{C} & -1.274294 & -0.04362 & 0.480962 \\ \mathrm{C} & -0.348147 & 1.370098 & -0.059321 \\ \mathrm{C} & 0.116 & 0.011416 & -0.277305 \\ \mathrm{C} & 1.344872 & -0.521127 & 0.448686 \\ \mathrm{H} & -3.39234 & -0.497591 & 0.249738 \\ \mathrm{H} & -2.266804 & -1.707051 & -0.434004 \\ \mathrm{H} & -2.546377 & -0.170636 & -1.29131 \\ \mathrm{H} & -1.077612 & -0.392667 & 1.506017 \\ \mathrm{H} & -1.605612 & 1.050448 & 0.761039 \\ \mathrm{H} & 0.07236 & 1.963245 & 0.763883 \\ \mathrm{H} & 1.248112 & -1.61678 & 0.534766 \\ \mathrm{H} & -0.014789 & -0.353089 & -1.304522 \\ \mathrm{H} & -0.892281 & 1.914146 & -0.840639 \\ \mathrm{C} & 2.638593 & -0.161183 & -0.294161 \\ \mathrm{H} & 3.505635 & -0.578933 & 0.237989 \\ \mathrm{H} & 2.646158 & -0.572923 & -1.315995 \\ \mathrm{H} & 2.78073 & 0.929626 & -0.362811 \\ \mathrm{H} & 1.366139 & -0.120825 & 1.478884 \\ & & & \\ \text { 8CA_5CB_tt_CC } & & \\ & & & \\ \mathrm{C} & -2.429567 & -0.630256 & -0.29771 \\ \mathrm{C} & -1.265799 & -0.036817 & 0.488019 \\ \mathrm{C} & -0.329569 & 1.369845 & -0.059374 \\ \mathrm{C} & 0.107329 & 0.014571 & -0.27951 \\ \mathrm{C} & 1.328711 & -0.527215 & 0.444363 \\ \mathrm{H} & -3.360959 & -0.525081 & 0.253591 \\ \mathrm{H} & -2.232109 & -1.687468 & -0.464529 \\ \mathrm{H} & -2.533885 & -0.140422 & -1.265097 \\ \mathrm{H} & -1.076767 & -0.390934 & 1.498198 \\ \mathrm{H} & -1.592311 & 1.04411 & 0.740292 \\ \mathrm{H} & 0.082667 & 1.946049 & 0.762085 \\ \mathrm{H} & 1.228349 & -1.611166 & 0.521306 \\ \mathrm{H} & -0.026159 & -0.343082 & -1.295442 \\ \mathrm{H} & -0.872798 & 1.912682 & -0.823865 \\ \mathrm{C} & 2.620077 & -0.164147 & -0.291008 \\ \mathrm{H} & 3.47759 & -0.58048 & 0.233904 \\ & 2.620117 & -0.564058 & -1.305142 \\ \mathrm{H} & 1.752754 & 0.91718 & -0.348109 \\ & & -0.133221 & 1.464129\end{array}$


1_7_PBE

$\begin{array}{llll}\text { C } & 0.776291 & -0.589882 & -0.316946 \\ \mathrm{C} & -0.673457 & 0.040537 & 0.207595 \\ \mathrm{H} & -0.963617 & -0.358484 & 1.241855 \\ \mathrm{C} & 1.917687 & 0.367221 & -0.57414 \\ \mathrm{H} & 2.190313 & 0.964196 & 0.307612 \\ \mathrm{H} & 2.796824 & -0.230022 & -0.865425 \\ \mathrm{H} & 1.691608 & 1.042725 & -1.412559 \\ \mathrm{C} & -0.582191 & 1.55384 & 0.44487 \\ \mathrm{H} & -1.565944 & 1.918668 & 0.776308 \\ \mathrm{H} & 0.156769 & 1.821667 & 1.213157 \\ \mathrm{H} & -0.318016 & 2.064888 & -0.492436 \\ \mathrm{C} & 0.488941 & -1.12485 & 0.975783 \\ \mathrm{H} & 0.005298 & -2.101218 & 1.09442 \\ \mathrm{H} & 0.938505 & -0.679377 & 1.872292 \\ \mathrm{C} & -1.798745 & -0.47867 & -0.701204 \\ \mathrm{H} & -1.663989 & -0.072707 & -1.715058 \\ \mathrm{H} & -1.80919 & -1.577515 & -0.763967 \\ \mathrm{H} & -2.773902 & -0.145294 & -0.316045 \\ \mathrm{H} & 0.544187 & -1.256704 & -1.155908\end{array}$

1_7_CC

$\begin{array}{llll}\text { C } & 0.760119 & -0.578349 & -0.323139\end{array}$

$\begin{array}{llll}\text { C } & -0.660469 & 0.033284 & 0.205485\end{array}$

$\begin{array}{llll}\mathrm{H} & -0.924507 & -0.378774 & 1.226263\end{array}$

$\begin{array}{llll}\text { C } & 1.903304 & 0.381236 & -0.574256\end{array}$

$\begin{array}{llll}\mathrm{H} & 2.146606 & 0.976291 & 0.302917\end{array}$

$\begin{array}{llll}\mathrm{H} & 2.780351 & -0.205318 & -0.845658\end{array}$

$\begin{array}{llll}\mathrm{H} & 1.674626 & 1.045224 & -1.405528\end{array}$

$\begin{array}{llll}\text { C } & -0.590464 & 1.542697 & 0.452272\end{array}$

$\begin{array}{llll}\mathrm{H} & -1.565015 & 1.881299 & 0.799663\end{array}$

$\begin{array}{llll}\mathrm{H} & 0.150846 & 1.805042 & 1.203915\end{array}$

$\begin{array}{llll}\mathrm{H} & -0.352411 & 2.05876 & -0.475518\end{array}$

$\begin{array}{llll}\text { C } & 0.507852 & -1.129377 & 0.961493\end{array}$

$\begin{array}{llll}\mathrm{H} & 0.029837 & -2.094438 & 1.077895\end{array}$

$\begin{array}{llll}\mathrm{H} & 0.950574 & -0.685427 & 1.846301\end{array}$

$\begin{array}{llll}\text { C } & -1.791182 & -0.478352 & -0.693217\end{array}$

$\begin{array}{llll}\mathrm{H} & -1.66721 & -0.067662 & -1.694053\end{array}$

$\begin{array}{llll}H & -1.789407 & -1.565894 & -0.758638\end{array}$

$\begin{array}{llll}\mathrm{H} & -2.751733 & -0.154943 & -0.296371\end{array}$

$\begin{array}{llll}\text { H } & 0.542481 & -1.240989 & -1.153005\end{array}$ 
3_1_PBE

$\begin{array}{llll}\mathrm{C} & 1.771989 & -0.399902 & -0.743076 \\ \mathrm{H} & 1.754769 & 0.085947 & -1.730458 \\ \mathrm{H} & 1.794109 & -1.491147 & -0.891495 \\ \mathrm{H} & 2.705791 & -0.104656 & -0.240105 \\ \mathrm{C} & 0.576482 & 0.052199 & 0.091931 \\ \mathrm{C} & -0.79602 & -0.646063 & -0.322368 \\ \mathrm{H} & -0.574883 & -1.372762 & -1.117106 \\ \mathrm{H} & -1.057746 & -1.445072 & 0.521009 \\ \mathrm{C} & 0.504734 & 1.556622 & 0.328355 \\ \mathrm{H} & -0.314403 & 1.855354 & 0.997782 \\ \mathrm{H} & 1.450928 & 1.885467 & 0.785031 \\ \mathrm{H} & 0.394929 & 2.092789 & -0.626515 \\ \mathrm{C} & -2.057953 & 0.196997 & -0.443875 \\ \mathrm{H} & -2.917919 & -0.437903 & -0.700709 \\ \mathrm{H} & -2.291975 & 0.743053 & 0.480796 \\ \mathrm{H} & -1.912153 & 0.933587 & -1.248708 \\ \mathrm{C} & 0.131385 & -0.840709 & 1.164719 \\ \mathrm{H} & 0.596322 & -1.827326 & 1.290144 \\ \mathrm{H} & -0.411473 & -0.432199 & 2.026216\end{array}$

\section{3_1_CC}

$\begin{array}{llll}\mathrm{C} & 1.753791 & -0.37966 & -0.758819 \\ \mathrm{H} & 1.699853 & 0.095629 & -1.737379 \\ \mathrm{H} & 1.78399 & -1.460488 & -0.89695 \\ \mathrm{H} & 2.68036 & -0.066792 & -0.279078 \\ \mathrm{C} & 0.568451 & 0.050213 & 0.094421 \\ \mathrm{C} & -0.788738 & -0.660699 & -0.311591 \\ \mathrm{H} & -0.570984 & -1.365909 & -1.108804 \\ \mathrm{H} & -1.054067 & -1.428849 & 0.52002 \\ \mathrm{C} & 0.483977 & 1.549899 & 0.334068 \\ \mathrm{H} & -0.329295 & 1.826392 & 1.001397 \\ \mathrm{H} & 1.420171 & 1.880783 & 0.781973 \\ \mathrm{H} & 0.359914 & 2.07612 & -0.611246 \\ \mathrm{C} & -2.044729 & 0.19364 & -0.44191 \\ \mathrm{H} & -2.883552 & -0.434171 & -0.734145 \\ \mathrm{H} & -2.293937 & 0.692684 & 0.491729 \\ \mathrm{H} & -1.884669 & 0.948053 & -1.209358 \\ \mathrm{C} & 0.165519 & -0.836613 & 1.165541 \\ \mathrm{H} & 0.617554 & -1.816777 & 1.270387 \\ \mathrm{H} & -0.374977 & -0.447362 & 2.021192\end{array}$




\section{Triple Shifts}

$\begin{array}{llll}\text { 2_2_Bu_gl_PBE } & & \\ \text { C } & -1.779883 & -0.409043 & -0.45217 \\ \text { C } & -0.296505 & 0.353226 & 0.580102 \\ \text { C } & -0.945226 & -1.379851 & 0.057152 \\ \text { H } & -1.135803 & -1.840845 & 1.030922 \\ \text { H } & -0.186298 & -1.845891 & -0.577214 \\ \text { H } & -1.679965 & -0.072951 & -1.487925 \\ \text { H } & -2.645081 & -0.044453 & 0.110136 \\ \text { H } & -0.781364 & 0.407774 & 1.564311 \\ \text { C } & -0.393816 & 1.696751 & -0.166129 \\ \text { H } & 0.023813 & 1.651871 & -1.181794 \\ \text { H } & -1.407364 & 2.1184 & -0.202671 \\ \text { H } & 0.234526 & 2.390087 & 0.421368 \\ \text { C } & 1.17364 & -0.123269 & 0.69796 \\ \text { H } & 1.23951 & -0.998784 & 1.361812 \\ \text { H } & 1.622945 & 0.711451 & 1.271382 \\ \text { C } & 1.947801 & -0.34775 & -0.619175 \\ \text { H } & 2.786616 & 0.355986 & -0.715301 \\ \text { H } & 2.370281 & -1.362205 & -0.6645 \\ \text { H } & 1.32212 & -0.210822 & -1.51697 \\ \text { 2_2_Bu_gr_PBE } & & \\ & & & \\ \text { C } & -1.536651 & 1.004745 & 0.199766 \\ \text { C } & -0.29667 & -0.344082 & -0.340543 \\ \text { C } & -0.329379 & 1.628275 & -0.027654 \\ \text { H } & -0.071968 & 2.046264 & -1.005602 \\ \text { H } & 0.364594 & 1.819244 & 0.794552 \\ \text { H } & -1.843558 & 0.741807 & 1.216263 \\ \text { H } & -2.305735 & 0.971047 & -0.57691 \\ \text { H } & -0.171156 & -0.204529 & -1.424409 \\ \text { C } & -1.33926 & -1.463363 & -0.078785 \\ \text { H } & -1.491918 & -1.647826 & 0.994047 \\ \text { H } & -2.307087 & -1.311909 & -0.574311 \\ \text { H } & -0.880016 & -2.36628 & -0.518514 \\ \text { C } & 1.018753 & -0.625083 & 0.403901 \\ \text { H } & 0.881196 & -0.441771 & 1.485004 \\ \text { C } & 2.283666 & 0.047611 & -0.137461 \\ \text { H } & 2.311865 & 1.136673 & 0.027321 \\ \text { H } & 3.166269 & -0.371922 & 0.367764 \\ \text { H } & 2.404763 & -0.138017 & -1.216872 \\ \text { H } & 1.13999 & -1.721396 & 0.316314\end{array}$




$\begin{array}{llll}\text { 2_2_Bu_t_PBE } & & \\ \text { C } & -1.96972 & 0.165975 & -0.222355 \\ \text { C } & -0.127463 & 0.168207 & 0.361114 \\ \text { C } & -1.648398 & -1.127989 & 0.119856 \\ \text { H } & -1.8059 & -1.510948 & 1.133059 \\ \text { H } & -1.319202 & -1.842784 & -0.638919 \\ \text { H } & -1.949612 & 0.486752 & -1.267923 \\ \text { H } & -2.456966 & 0.83211 & 0.494926 \\ \text { H } & -0.182467 & 0.075896 & 1.455591 \\ \text { C } & 0.039174 & 1.652566 & -0.030478 \\ \text { H } & 0.097599 & 1.79701 & -1.118854 \\ \text { H } & -0.7198 & 2.32053 & 0.397272 \\ \text { H } & 1.009427 & 1.944606 & 0.406228 \\ \text { C } & 0.93154 & -0.748105 & -0.281688 \\ \text { H } & 0.814243 & -1.782254 & 0.077992 \\ \text { H } & 0.791215 & -0.759181 & -1.377317 \\ \text { C } & 2.360868 & -0.279355 & 0.057527 \\ \text { H } & 3.066641 & -1.052393 & -0.281871 \\ \text { H } & 2.631703 & 0.658784 & -0.446966 \\ \text { H } & 2.507115 & -0.155924 & 1.142927\end{array}$

\section{2_2_Me_g_PBE}

$\begin{array}{llll}\text { C } & -0.93669 & -0.883907 & 0.000001 \\ \mathrm{C} & 0.433754 & -0.181241 & 0 \\ \mathrm{C} & 0.585256 & 1.052639 & -0.864038 \\ \mathrm{H} & -0.316443 & 1.444765 & -1.349896 \\ \mathrm{H} & 0.701627 & 2.010294 & 0.000001 \\ \mathrm{C} & 0.585257 & 1.05264 & 0.864038 \\ \mathrm{H} & -0.316442 & 1.444764 & 1.349897 \\ \mathrm{H} & 1.552041 & 1.207288 & 1.35792 \\ \mathrm{H} & 1.55204 & 1.207289 & -1.357921 \\ \mathrm{H} & -0.945539 & -1.549589 & 0.880224 \\ \mathrm{C} & 1.624106 & -1.138082 & 0 \\ \mathrm{H} & 1.5851 & -1.785088 & 0.889435 \\ \mathrm{H} & 1.5851 & -1.785089 & -0.889435 \\ \mathrm{H} & 2.590422 & -0.609344 & -0.000001 \\ \mathrm{C} & -2.192279 & -0.008535 & 0 \\ \mathrm{H} & -3.082333 & -0.655309 & 0.000001 \\ \mathrm{H} & -2.278229 & 0.629259 & 0.896339 \\ \mathrm{H} & -2.278229 & 0.629257 & -0.896341 \\ \mathrm{H} & -0.945539 & -1.54959 & -0.880222\end{array}$




\begin{tabular}{|c|c|c|c|}
\hline \multicolumn{4}{|c|}{ 2_2_Me_t_PBE } \\
\hline C & -0.998168 & -0.445266 & -0.644315 \\
\hline C & 0.320173 & 0.139823 & -0.111859 \\
\hline $\mathrm{C}$ & 0.990066 & -0.659366 & 0.983686 \\
\hline $\mathrm{H}$ & 0.54122 & -1.622692 & 1.256396 \\
\hline $\mathrm{H}$ & 2.038075 & -1.154867 & 0.387716 \\
\hline $\mathrm{C}$ & 1.583241 & -0.517242 & -0.635533 \\
\hline $\mathrm{H}$ & 1.462179 & -1.393774 & -1.284334 \\
\hline $\mathrm{H}$ & 2.460999 & 0.122922 & -0.787521 \\
\hline $\mathrm{H}$ & 1.541574 & -0.109843 & 1.756426 \\
\hline $\mathrm{C}$ & -2.201044 & -0.186583 & 0.269857 \\
\hline $\mathrm{H}$ & -2.42385 & 0.886705 & 0.368606 \\
\hline $\mathrm{H}$ & -3.096722 & -0.667391 & -0.150193 \\
\hline $\mathrm{H}$ & -2.0478 & -0.600404 & 1.280778 \\
\hline $\mathrm{H}$ & -1.176654 & -0.005353 & -1.640649 \\
\hline $\mathrm{C}$ & 0.377111 & 1.657937 & 0.037948 \\
\hline $\mathrm{H}$ & 0.169611 & 2.136658 & -0.931053 \\
\hline $\mathrm{H}$ & -0.383597 & 1.998524 & 0.75507 \\
\hline $\mathrm{H}$ & 1.360189 & 2.006143 & 0.39219 \\
\hline $\mathrm{H}$ & -0.873498 & -1.532445 & -0.802138 \\
\hline \multicolumn{4}{|c|}{ 6CB_6CB_Bu_C1_PBE } \\
\hline $\mathrm{C}$ & -1.990327 & -0.488405 & -0.533501 \\
\hline $\mathrm{C}$ & -0.455482 & -0.506638 & 0.390801 \\
\hline $\mathrm{H}$ & -0.431603 & -0.36188 & 1.49017 \\
\hline $\mathrm{C}$ & -1.853219 & 0.576321 & 0.359394 \\
\hline $\mathrm{H}$ & -1.441423 & 1.5347 & 0.032461 \\
\hline $\mathrm{H}$ & -2.382893 & 0.567623 & 1.316575 \\
\hline $\mathrm{H}$ & -0.595115 & -1.608237 & 0.356445 \\
\hline $\mathrm{H}$ & -2.619967 & -1.349377 & -0.28741 \\
\hline $\mathrm{H}$ & -1.659112 & -0.398377 & -1.57251 \\
\hline $\mathrm{C}$ & 0.816104 & -0.042881 & -0.339967 \\
\hline C & 1.169698 & 1.431723 & -0.097793 \\
\hline $\mathrm{H}$ & 1.255536 & 1.6567 & 0.978753 \\
\hline $\mathrm{H}$ & 2.144526 & 1.653835 & -0.55722 \\
\hline $\mathrm{H}$ & 0.449397 & 2.134139 & -0.549173 \\
\hline $\mathrm{H}$ & 0.671377 & -0.206809 & -1.423502 \\
\hline $\mathrm{C}$ & 1.953989 & -0.972525 & 0.132793 \\
\hline $\mathrm{H}$ & 1.732211 & -2.034988 & -0.060724 \\
\hline $\mathrm{H}$ & 2.880719 & -0.723449 & -0.405157 \\
\hline $\mathrm{H}$ & 2.151772 & -0.849448 & 1.210933 \\
\hline
\end{tabular}




$\begin{array}{llll}\text { 6CB_6CB_Bu_CS_PBE } & & \\ \text { C } & 1.715325 & 0.697234 & -0.36069 \\ \mathrm{C} & 0.564654 & 0.000114 & 0.822706 \\ \mathrm{H} & 0.865395 & -0.849963 & 1.467828 \\ \mathrm{C} & 1.715379 & -0.697217 & -0.360455 \\ \mathrm{H} & 1.072733 & -1.259663 & -1.043882 \\ \mathrm{H} & 2.508762 & -1.255448 & 0.145943 \\ \mathrm{H} & 0.865265 & 0.850501 & 1.467489 \\ \mathrm{H} & 2.508648 & 1.255685 & 0.145558 \\ \mathrm{H} & 1.072628 & 1.25942 & -1.044289 \\ \mathrm{C} & -0.929284 & -0.00003 & 0.453091 \\ \mathrm{C} & -1.391361 & -1.276285 & -0.265884 \\ \mathrm{H} & -1.047965 & -2.192805 & 0.24124 \\ \mathrm{H} & -2.490922 & -1.308284 & -0.289989 \\ \mathrm{H} & -1.05793 & -1.304806 & -1.31831 \\ \mathrm{H} & -1.397991 & -0.000084 & 1.457757 \\ \mathrm{C} & -1.391559 & 1.276171 & -0.265842 \\ \mathrm{H} & -2.491127 & 1.308105 & -0.289812 \\ \mathrm{H} & -1.048159 & 2.192724 & 0.241217 \\ \mathrm{H} & -1.058258 & 1.304699 & -1.318308 \\ & & & \\ \text { 6CB_6CB_Me_g_PBE } & & \\ & & & \\ \mathrm{C} & 0.840363 & 0.097935 & -0.459431 \\ \mathrm{C} & -0.575774 & -0.502552 & -0.427249 \\ \mathrm{C} & -1.348719 & -0.555723 & 0.86059 \\ \mathrm{H} & -0.943884 & 0.010257 & 1.709098 \\ \mathrm{H} & -2.345831 & 0.263946 & 0.628228 \\ \mathrm{C} & -1.803127 & 0.373868 & -0.528011 \\ \mathrm{H} & -1.638673 & 1.457229 & -0.474164 \\ \mathrm{H} & -2.648117 & 0.002751 & -1.11923 \\ \mathrm{H} & -1.957271 & -1.442643 & 1.071169 \\ \mathrm{H} & -0.675835 & -1.424022 & -1.012512 \\ \mathrm{H} & 1.836677 & -0.964575 & 0.037652 \\ \mathrm{H} & 1.747965 & -1.907831 & -0.524871 \\ \mathrm{H} & 2.868063 & -0.601345 & -0.083058 \\ \mathrm{H} & 1.686277 & -1.186531 & 1.108229 \\ \mathrm{H} & 1.044603 & 0.290683 & -1.528925 \\ \mathrm{H} & 0.994662 & 1.420795 & 0.303432 \\ & 2.025335 & 1.787211 & 0.183684 \\ & 0.338295 & 2.225325 & -0.070054 \\ & 0.834581 & 1.306477 & 1.390514\end{array}$




$\begin{array}{llll}\text { 6CB_6CB_Me_t_PBE } & & \\ & & & \\ \mathrm{C} & -0.770839 & -0.00001 & -0.305631 \\ \mathrm{H} & -0.548201 & -0.000043 & -1.39234 \\ \mathrm{C} & 0.568824 & 0.000017 & 0.438257 \\ \mathrm{C} & 1.690318 & -0.867584 & -0.070198 \\ \mathrm{H} & 1.53175 & -1.360205 & -1.038424 \\ \mathrm{H} & 2.554917 & -0.000021 & -0.497993 \\ \mathrm{C} & 1.690317 & 0.867582 & -0.070258 \\ \mathrm{H} & 1.531752 & 1.360131 & -1.038521 \\ \mathrm{H} & 2.345662 & 1.358361 & 0.659037 \\ \mathrm{H} & 2.345658 & -1.358315 & 0.659135 \\ \mathrm{H} & 0.487889 & 0.000054 & 1.532281 \\ \mathrm{C} & -1.558035 & 1.274605 & 0.035987 \\ \mathrm{H} & -1.006566 & 2.190974 & -0.233182 \\ \mathrm{H} & -2.511191 & 1.287159 & -0.513384 \\ \mathrm{H} & -1.794625 & 1.319713 & 1.112157 \\ \mathrm{C} & -1.558031 & -1.274606 & 0.036067 \\ \mathrm{H} & -1.794621 & -1.319647 & 1.112239 \\ \mathrm{H} & -2.511185 & -1.287198 & -0.513305 \\ \mathrm{H} & -1.006558 & -2.190989 & -0.233045 \\ & & & \\ \text { 8CB_8CB_Bu_g_PBE } & & \\ & & & \\ \mathrm{C} & -1.812463 & 0.955085 & -0.061093 \\ \mathrm{C} & -0.97891 & -0.538359 & -0.575235 \\ \mathrm{H} & -1.547288 & -1.475725 & -0.741824 \\ \mathrm{C} & -2.236825 & -0.176633 & 0.637195 \\ \mathrm{H} & -1.768473 & -0.461988 & 1.58379 \\ \mathrm{H} & -3.185847 & -0.662196 & 0.389133 \\ \mathrm{C} & 0.437884 & -0.741982 & -0.040861 \\ \mathrm{H} & 0.407857 & -1.134387 & 0.990214 \\ \mathrm{H} & 0.850246 & -1.564083 & -0.65574 \\ \mathrm{H} & -1.003912 & 1.584309 & 0.321886 \\ \mathrm{H} & 1.381945 & 0.467149 & -0.135564 \\ \mathrm{H} & 1.048431 & 1.271341 & 0.547853 \\ \mathrm{H} & 1.345349 & 0.884165 & -1.159058 \\ \mathrm{C} & 2.824939 & 0.087967 & 0.219281 \\ \mathrm{H} & 3.48323 & 0.967033 & 0.160171 \\ & 2.895113 & -0.31477 & 1.242794 \\ \mathrm{H} & -2.219074 & -0.673506 & -0.472933 \\ & -1.023301 & -0.107135 & -1.596291\end{array}$




$\begin{array}{llll}\text { 8CB_8CB_Bu_t_PBE } & & \\ & & & \\ \mathrm{C} & 2.306766 & -0.698234 & 0.215543 \\ \mathrm{C} & 0.843784 & -0.000509 & -0.521314 \\ \mathrm{H} & 0.899106 & 0.845257 & -1.237572 \\ \mathrm{C} & 2.307037 & 0.698524 & 0.214091 \\ \mathrm{H} & 1.920993 & 1.261734 & 1.068764 \\ \mathrm{H} & 2.900196 & 1.25395 & -0.5189 \\ \mathrm{C} & -0.406786 & 0.000513 & 0.352088 \\ \mathrm{H} & -0.415255 & -0.886921 & 1.007591 \\ \mathrm{H} & -0.415276 & 0.889554 & 1.005404 \\ \mathrm{H} & 1.920639 & -1.259491 & 1.071457 \\ \mathrm{C} & -1.670264 & -0.00061 & -0.533249 \\ \mathrm{H} & -1.653994 & -0.887237 & -1.193756 \\ \mathrm{H} & -1.654083 & 0.884423 & -1.195895 \\ \mathrm{C} & -2.953357 & 0.000316 & 0.305197 \\ \mathrm{H} & -3.838155 & -0.000448 & -0.348353 \\ \mathrm{H} & -3.012919 & -0.890852 & 0.950744 \\ \mathrm{H} & -3.012956 & 0.892945 & 0.948716 \\ \mathrm{H} & 2.899784 & -1.255366 & -0.516261 \\ \mathrm{H} & 0.898847 & -0.847552 & -1.236072 \\ & & & \\ \text { 8CB_8CB_Me_g_PBE } & & \\ & & & \\ \mathrm{C} & 0.967168 & -0.110267 & 0.491598 \\ \mathrm{C} & 2.234327 & -0.679864 & -0.090245 \\ \mathrm{H} & 2.751638 & 0.338516 & -0.71051 \\ \mathrm{C} & 1.719254 & 0.968386 & -0.249115 \\ \mathrm{H} & 1.315457 & 1.267356 & -1.224971 \\ \mathrm{H} & 2.271105 & 1.714663 & 0.334462 \\ \mathrm{H} & 1.014308 & 0.014021 & 1.579869 \\ \mathrm{H} & 3.079847 & -0.866453 & 0.582497 \\ \mathrm{H} & 2.12931 & -1.315875 & -0.97861 \\ \mathrm{C} & -0.381986 & -0.565613 & -0.051315 \\ \mathrm{H} & -0.312435 & -0.708234 & -1.146698 \\ \mathrm{C} & -1.515492 & 0.417407 & 0.286617 \\ \mathrm{H} & -1.555127 & 0.56406 & 1.381394 \\ \mathrm{H} & -1.287787 & 1.408069 & -0.150689 \\ \mathrm{C} & -2.875466 & -0.073864 & -0.220495 \\ \mathrm{H} & -3.146117 & -1.040951 & 0.233348 \\ \mathrm{H} & -3.667524 & 0.646351 & 0.032561 \\ \mathrm{H} & -2.876678 & -0.201192 & -1.315513 \\ & -0.602824 & -1.557447 & 0.380591\end{array}$




\begin{tabular}{|c|c|c|c|}
\hline C & -1.098973 & 0.693763 & 0.000432 \\
\hline C & -1.775308 & -0.338933 & -0.866069 \\
\hline $\mathrm{H}$ & -2.27625 & -1.163148 & -0.00087 \\
\hline C & -1.775048 & -0.339851 & 0.865781 \\
\hline $\mathrm{H}$ & -1.126026 & -1.077029 & 1.354795 \\
\hline $\mathrm{H}$ & -2.716611 & -0.067368 & 1.356165 \\
\hline $\mathrm{H}$ & -1.634997 & 1.649635 & 0.000913 \\
\hline $\mathrm{H}$ & -2.716821 & -0.06554 & -1.356037 \\
\hline $\mathrm{H}$ & -1.126337 & -1.075355 & -1.356311 \\
\hline C & 0.42489 & 0.831536 & 0.000329 \\
\hline $\mathrm{H}$ & 0.688816 & 1.443338 & -0.880204 \\
\hline C & 1.254059 & -0.460571 & -0.000061 \\
\hline $\mathrm{H}$ & 1.01628 & -1.076781 & 0.888677 \\
\hline $\mathrm{H}$ & 1.015946 & -1.076471 & -0.88891 \\
\hline C & 2.760495 & -0.163648 & -0.000204 \\
\hline $\mathrm{H}$ & 3.052403 & 0.415215 & 0.89057 \\
\hline $\mathrm{H}$ & 3.341518 & -1.09747 & 0.000255 \\
\hline $\mathrm{H}$ & 3.052453 & 0.414405 & -0.891481 \\
\hline $\mathrm{H}$ & 0.688937 & 1.442799 & 0.881189 \\
\hline
\end{tabular}




\section{Additional References}

29. (a) Kirchen, R. P.; Sorensen, T. S. J. Am. Chem. Soc. 1979, 101, 3240. (b) Kirchen, R. P.; Ranganayakulu, K.; Singh, B. P.; Sorensen, T. S.; Can. J. Chem. 1981, 59, 2173. (c) McMurry, J. E.; Lectka, T.; Hodge, C. N. J. Am. Chem. Soc. 1989, 111, 8867. (d) Saunders, M.; Jimenez-Vazquez, H. A. Chem. Rev. 1991, 91, 375.

30. (a) Boronat, M.; Viruela, P.; Corma, A. J. Phys. Chem. 1996, 100, 633. (b) ibid. 16514. 31. Evans, M. G.; Polanyi, M., J. Chem. Soc., Faraday Trans. 1936, 32, 1340. 